\title{
Homogenization of Nonlinearly Elastic Materials, Microscopic Bifurcation and Macroscopic Loss of Rank-One Convexity
}

\author{
Giuseppe Geymonat, Stefan Müller \& Nicolas Triantafylldis
}

Communicated by R. V. KoHN

\section{Table of Contents}

1. Introduction . . . . . . . . . . . . . . . . . . . 232

2. Homogenization of Nonconvex Integral Functionals. . . . . . . . . . 235

3. The Linearized System (I): Results . . . . . . . . . . . . . . . . . . . 238

3.1. Convergence of solutions for $\Lambda>0$. . . . . . . . . . . . . . . 238

3.2. $\Gamma$-convergence for $\Lambda \geqq 0$. . . . . . . . . . . . . . . . . . . . 241

3.3. Summary of the coercivity constants . . . . . . . . . . . . . . 243

3.4. Layered materials . . . . . . . . . . . . . . . . . . . . . . . 243

4. The Linearized System (II): Proofs . . . . . . . . . . . . . . . . . 245

4.1. Preliminary results. . . . . . . . . . . . . . . . . . 245

4.2. Proof of Theorem 3.3 . . . . . . . . . . . . . . . . . . . . . . 248

4.3. Proof of Theorem 3.1 . . . . . . . . . . . . . . . . . . . . . . 251

4.4. Proof of Theorem 3.4(i) when $A>0$. . . . . . . . . . . . . . . . 251

4.5. Proof of Theorem 3.4 (i) when $A=0$ and $A_{6}>0$. . . . . . . . . . 252

4.6. Proof of Theorem 3.4(ii) . . . . . . . . . . . . . . . . . . . 255

4.7. Proof of Theorem 3.4(iii) . . . . . . . . . . . . . . . . . 256

4.8. Proof of Proposition 3.6. . . . . . . . . . . . . . . . . . . . . 257

4.9. Proof of Theorem 3.7 (for stratified materials) . . . . . . . . . . . 257

5. Nonlinear Homogenization and Loss of Strong Ellipticity. . . . . . . . . 258

5.1. General remarks. . . . . . . . . . . . . . . . . . . 258

5.2. Main results. . . . . . . . . . . . . . . . . . . . . . 259

5.3. A weakened hypothesis . . . . . . . . . . . . . . . . 263

5.4. Convex integrands . . . . . . . . . . . . . . . . . . . . 265

6. Application to a Layered Composite . . . . . . . . . . . . . . . . . . 269

6.1. Model - General considerations, calculation of $M$. . . . . . . . . 270

6.2. Calculation of $\Lambda_{1}^{s}, \Lambda_{6}^{s}$. . . . . . . . . . . . . . . . . . 272

6.3. Calculation of $\Lambda_{5}^{s}(\lambda)$. . . . . . . . . . . . . . . . . . . 278

6.4. Calculation of $\alpha_{M}^{s}(\lambda)$. . . . . . . . . . . . . . . . . . . . . . . 280

6.5. Calculation of the critical stretch ratios $\lambda_{c}^{+}, \lambda_{c}^{-}$and $\lambda_{h}^{+}, \lambda_{h}^{-}$. . . . 281

6.6. Example for a particular energy density $W$. . . . . . . . . . 283

References... . . . . . . . . . . . . . . . . . 288 


\section{Introduction}

A fundamental problem in the study of composite materials (such as fiberreinforced materials, layered materials, honeycombs, foams, etc....) is the determination of their macroscopic (or "average") behavior as a function of their microstructure. The calculation of these macroscopic properties is called the homogenization (or "averaging") problem for the composite and has attracted a great deal of attention both in the engineering as well as the mathematics literature.

The first homogenization problems to be addressed in the engineering literature were concerned with linearly elastic composites. For the simplest possible microstructures, namely the periodic ones (obtained by the infinite repetition in space of a fundamental unit cell), the homogenized elastic moduli can be calculated exactly (see HILL [H 63]). Since exact calculations are no longer possible for the more complicated case of aperiodic microstructures, there are two philosophically different approaches to the problem. On one hand, approximate methods have been developed to calculate the homogenized moduli of aperiodic composites. Perhaps the most popular of these methods are the several variations of the "self-consistent" scheme developed initially by HILL [H 65] and KRÖNER [K 67] and subsequently employed by many researchers in this field. On the other hand, a successful approach has also been developed to construct bounds for the macroscopic moduli of arbitrary linearly elastic composites. Although the simplest arithmetic and harmonic bounds have been known for about a century, interest in this approach has been revived with the work of HASHIN \& SHTRIKMAN [HS 62] and continues strongly to date. For a brief review of the aforementioned approaches in the engineering literature see WIILIS [W 81] or HILYARD [HI 82] and references quoted therein.

In parallel to the above developments, a rigorous mathematic approach to the homogenization problem in linear elastic solids has been developed in the early sixties and seventies. A justification of HnL's results for periodic media has been given via the asymptotic expansion method proposed by SANCHEZPALENCIA [SP 74]. The considerable progress that has been made in the mathematical study of various aspects of the homogenization in linearly elastic composites can be reviewed in Benssoussan, Lions \& Papanicolaou [BLP 78], Sanchez-Palencia [SP 80] and Kohn \& Strang [KS 86]. In addition to these results in linear elasticity, one should also mention the more recent generalizations in linear viscoelastic (see [SP 80]) and thermoelastic periodic composites (see FrancFort [F 83]).

The study of nonlinear composite solids presents considerable additional diffculties. The main difference with the linear case is that the macroscopic behavior of the nonlinear composite can be of a nature completely different (and often unknown) from the microscopic behavior of its constituents. As a result, the majority of the numerous engineering papers on this subject involve a variety of approximations difficult to verify. Consequently, the engineering studies in this area have not fostered an analogous development in the mathematics literature as for the linear case. The only apparent deviation 
from this trend seems to be the case of nonlinearly elastic composites, the topic of interest in the present work.

The simplest, and yet practically meaningful and theoretically tractable type of nonlinear composites, are the periodic nonlinearly elastic composites. In the engineering literature, ABEYARATNE \& TRIANTAFYlLIDIS [AT 84], motivated by the desire to understand why the experimentally produced energy density functions for a certain type of foam rubber show the possibility of a shear band (in mathematical terms the energy density loses its rank-one convexity), have studied numerically the behavior of an elastomeric composite with periodic holes. It was found that although the matrix material is polyconvex in the sense of BALL [B 77] and hence always rank-one convex, the homogenized incremental (or linearized) moduli of the composite lose their rank-one convexity at adequately high macroscopic strains.

In an effort to further understand the reasons for such a behavior, TRIANTAFYLLIDIS \& MAKER [TM 85] have studied the bifurcation problem of a finitely strained layered nonlinearly elastic composite. They found that there exists an intimate connection between bifurcation at the microscopic level and loss of rank-one convexity at the macroscopic level. More precisely, they showed that bifurcation of the composite at a wavelength much larger than the unit cell size corresponds to the loss of rank-one convexity in the homogenized incremental moduli. As it turns out, this very interesting connection between microscopic and macroscopic instability mechanisms in nonlinearly layered composites is a much more general property that holds for all nonlinearly elastic periodic composites and will be proved (under suitable hypotheses) in Section 5.

One should also mention the work of TALBOT \& WiLLIS [TW 87] on bounding theorems for aperiodic nonlinearly elastic composites, in a spirit that follows the corresponding work for the linear case.

On the mathematical side, the study of problems in nonlinearly elastic composites has greatly advanced through the introduction of the notion of $\Gamma$ convergence by DEGIoRGI [D 75]. Using that notion BRAIDES [Br 85] and MÜLLER [Mü 87] obtained results relevant in nonlinear elasticity. They study materials with a periodic microstructure which are characterized by a stored energy density $W(x / \varepsilon, F)$ depending on the local position $x / \varepsilon$ and the deformation gradient $F, \varepsilon$ being the period of the structure. They find that in the limit $\varepsilon \rightarrow 0$ the material is described by a (homogeneous) stored energy density $\bar{W}(F)$ depending only on the deformation gradient (and not explicitly on the position) and they give an abstract formula for $\bar{W}$ (see Section 2 below).

One fundamental difficulty encountered in nonlinear elasticity is that $W$ is not a convex function of the deformation gradient. In fact, the homogenization result for convex integrands (see MARCELLINI [Ma 78]) differs substantially from that for nonconvex integrands (see Section 2), this difference being related to possible instabilities such as buckling on the microscale (see Sections 1 and 4 in [Mü 87]).

The purpose of the present paper is twofold. First, we study the relation between microscopic instabilities (i.e., the existence of nontrivial solutions to the linearized homogeneous problem) and the corresponding macroscopic in- 
stabilities (i.e., the failure of strong ellipticity for the homogenized incremental moduli). One of the main results is that long wavelength instabilities for the linearized problem lead to loss of rank-one convexity of the homogenized energy density (see Theorems 3.4(iii) and 5.1 (ii)).

Secondly, we investigate the commutability issue between the homogenization and the linearization operations in nonlinear periodic composites. For convex $\bar{W}$ a rigorous derivation of that formula is included, extending some previous results of ATTOUCH [A 84] (see also [FM 91]). This result may be stated as the fact that homogenization and linearization commute.

Some of the results of this paper have been announced in [GMT 90].

The outline of the paper is as follows: In Section 2, we review the notion of $\Gamma$-convergence and the homogenization results in nonlinear elasticity.

Sections 3 and 4 concern homogenization results in linearized elasticity; in fact, they apply to more general symmetric second-order strongly elliptic systems $-\operatorname{div} \llbracket(x) \nabla \boldsymbol{u}=\boldsymbol{f}$ in divergence form. The main difficulty is that in general the 4-tensor $\mathbb{L}(x)$ is not pointwise coercive (i.e., $\mathbb{L}(x)$ does not satisfy $(\mathbb{L}(x) G, G) \geqq C|G|^{2}, C>0$ for all $N \times N$ matrices $\left.G\right)$ so that solutions may not be a priori bounded in $H^{1}$ (see LEDRET [LD 87]). This difficulty is overcome by introducing the quantity

$$
\Lambda=\inf \left\{\int_{\mathbb{R}^{N}}(\amalg(\mathrm{x}) \nabla \boldsymbol{u}, \nabla \boldsymbol{u}) d x / \int_{\mathbb{R}^{N}}|\nabla \boldsymbol{u}|^{2} d x \mid \boldsymbol{u} \in \mathscr{D}\left(\mathbb{R}^{N}, \mathbb{R}^{N}\right)\right\},
$$

(see below for notations) which measures the overall coercivity. One result which is important in applications (cf. in particular Section 6.1) is that $\Lambda$ can equivalently be characterized in terms of Bloch waves $e^{i \omega \cdot x} p(x), p$ being periodic on the unit cube (see Lemma 4.2). Other measures of coercivity are also introduced and their properties are summarized in Section 3.3.

We show (in Theorem 3.1) that the equations have the usual homogenization limit if $\Lambda>0$, while $\Gamma$-convergence holds if $\Lambda=0$ (Theorem 3.4(i)). Moreover, if $\Lambda=0$, the whole space problem admits a non-trivial solution, and according to the character of that solution (Bloch wave or the longwave-length limit) the linearly homogenized problem retains or loses strong ellipticity (Theorem 3.4(ii), (iii) and Theorem 3.5).

In Section 5, we combine the results from the linear and the nonlinear theory and study in particular the question whether homogenization and linearization commute, i.e., whether the second derivatives of the nonlinearly homogenized energy density $\bar{W}$ can be obtained by studying the homogenized energy of an associated linear problem.

We show that under certain technical hypotheses (see (H1) and (H2)) $\bar{W}$ has the expected second-order Taylor expansion and that loss of strong ellipticity for the homogenization of the linear problem implies loss of ellipticity for $\bar{W}$ (see Theorem 5.1).

To complement these results we show (see Theorem 5.3) that for strictly convex $W$, with quadratic growth, homogenization and linearization do indeed commute and that no technical assumptions like $(\mathrm{H} 1)$ or $(\mathrm{H} 2)$ are required in that case. 
Section 6 contains an application of the general theory developed in Section 4 to the case of a layered nonlinearly elastic composite. The composite is under a state of plane strain with one of its principal stretch axes constantly aligned with the direction of lamination. For this problem one is able to calculate the quantities $\Lambda, \Lambda_{5}^{s}, \Lambda_{6}^{s}, \alpha_{M}^{s}$ as functions of the applied stretch ratio $\lambda$ in the lamination direction. One can also calculate for the given layered composite the critical values $\lambda_{c}^{+}$and $\lambda_{c}^{-}$corresponding to a first bifurcation in tension and compression of the composite, respectively, as well as $\lambda_{h}^{+}$and $\lambda_{h}^{-}$, which are the stretch ratios corresponding to the first loss of ellipticity in tension and compression, respectively. The application is completed by an example where all these quantities are calculated for a composite made of a nonlinear elastic material with a given energy density.

Notation. Vectors (with the exception of the current point of $\mathbb{R}^{N}$ ) and vectorvalued functions are represented by a boldface minuscule. Tensor of order 4 are represented by an outline majuscule. The matrix inner product of $C$ and $D$ is $(C, D)=$ trace $C \cdot{ }^{T} \bar{D}$, where $\bar{D}$ is the complex conjugate of $D$. By $H^{1, p}\left(\Omega ; \mathbb{R}^{N}\right)$ or $H^{1, p}\left(\Omega ; \mathbb{C}^{N}\right) \quad(1<p<\infty)$ we denote the usual Sobolev spaces of vector-valued functions on an open set $\Omega$, and we drop $p$ for $p=2$. Moreover $H_{i f}^{1, p}$ and $H_{k \neq}^{1, p}$ denote the spaces of all $H_{\mathrm{loc}}^{1, p}\left(\mathbb{R}^{N} ; \mathbb{R}^{N}\right)$ functions periodic on the unit cell $Y=[0,1]^{N}$ and on $k Y=[0, k]^{N}$, respectively, and $\mathscr{H}_{\sharp}^{1, p}$ and $\mathscr{H}_{k^{*} t}^{1, p}$ refer to the corresponding spaces of $\mathbb{C}^{N}$-valued functions. We use the symbol $\rightarrow$ to denote weak convergence. By $\mathbb{N}^{*}$ we denote the strictly positive integers.

\section{Homogenization of Nonconvex Integral Functionals}

Here we briefly review the results of [Mü 87] (cf. also [Br 85]). We consider a (hyper-) elastic material with a periodic microstructure described by the stored-energy density $W(x, F)$ depending on the position $x$ and the local deformation gradient $F$. We assume that $W$ is $Y$-periodic in $x$, where $Y=[0,1]^{N}$, $N \geqq 2$, and has polynomial growth in $F$, i.e.,

$$
\begin{gathered}
W(x+z, F)=W(x, F) \quad \forall z \in \mathbb{Z}^{N}, \\
c|F|^{p} \leqq W(x, F) \leqq C\left(1+|F|^{p}\right), \\
\left|\frac{\partial W}{\partial F}(x, F)\right| \leqq C\left(1+|F|^{p-1}\right),
\end{gathered}
$$

where $C, c>0, p>1$. Let $\varepsilon>0$ be the scale of the microstructure; then

$$
I^{\varepsilon}(\boldsymbol{u})=\int_{\Omega} W\left(\frac{x}{\varepsilon}, \nabla \boldsymbol{u}(x)\right) d x
$$

is the energy corresponding to a deformation $u: \Omega \rightarrow \mathbb{R}^{N}$ of a piece of material filling the region $\Omega$. As $\varepsilon \rightarrow 0$, i.e., as the microstructure becomes increasingly finer, we expect $I^{\varepsilon}$ to converge (in a sense to be defined below) to 
some homogenized functional

$$
I(\boldsymbol{u})=\int_{\Omega} \bar{W}(\nabla \boldsymbol{u}(x)) d x
$$

where the homogenized energy density $\bar{W}$ depends only on the deformation gradient and not explicitly on the position. A crucial point in passing from (2.4) to (2.5) is to define a suitable notion of convergence for the functionals $I^{\varepsilon}$. This problem was solved by DeGToRGI [D 75] by introducing the notion of $\Gamma$ convergence. The importance of this notion lies in the fact that, under appropriate technical hypotheses, it implies the convergence of minimizers which in our setting are solutions of the elastic equilibrium equations. $\Gamma$-convergence has been studied in a context much more general than ours; see DEGIORGI [D 79], DeGiorgi \& DalMaso [DD 83], DalMaso \& Modica [DM 81] and Аттолсн [A 84] for an overview. For our purpose the following definition is sufficient.

Definition 2.1. Let $\left\{I^{\varepsilon}\right\}_{\varepsilon>0}$ be a family of functionals on $H^{1, p}\left(\Omega ; \mathbb{R}^{N}\right)(1<p<\infty)$. We say that $\left\{I^{\varepsilon}\right\}_{\varepsilon>0}$ is $\Gamma$-convergent to a functional $I$, with respect to weak convergence of sequences in $H^{1, p}$ as $\varepsilon \rightarrow 0$ if the following conditions hold: (i) If $\boldsymbol{u}^{\varepsilon} \rightarrow \boldsymbol{u}$ (weakly in $H^{1, p}$ ) as $\varepsilon \rightarrow 0$, then

$$
\liminf _{\varepsilon \rightarrow 0} I^{\varepsilon}\left(\boldsymbol{u}^{\varepsilon}\right) \geqq I(\boldsymbol{u}) .
$$

(ii) For every $u \in H^{1, p}\left(\Omega ; \mathbb{R}^{N}\right)$ there is a sequence $\boldsymbol{u}^{\varepsilon} \rightarrow \boldsymbol{u}$ weakly in $H^{1, p}$ such that

$$
\lim _{\varepsilon \rightarrow 0} I^{\varepsilon}\left(\boldsymbol{u}^{\varepsilon}\right)=I(u) .
$$

One easily deduces the following result on the convergence of minimizers (for a proof see Aтtouch [A 84, pp. 39-41]).

Lemma 2.2. Assume that $\left\{I^{\varepsilon}\right\}_{\varepsilon>0}$ is $\Gamma$-convergent to $I$ as $\varepsilon \rightarrow 0$ and that $g$ is a weakly continuous functional on $H^{1, p}\left(\Omega ; \mathbb{R}^{N}\right)$. Let $\boldsymbol{u}^{\varepsilon}$ be an approximate minimizer of $I^{\varepsilon}+g$, i.e.,

$$
I^{\varepsilon}\left(\boldsymbol{u}^{\varepsilon}\right)+g\left(\boldsymbol{u}^{\varepsilon}\right)<\inf \left\{I^{\varepsilon}(\boldsymbol{u})+g(\boldsymbol{u}) \mid \boldsymbol{u} \in H^{1, p}\left(\Omega ; \mathbb{R}^{N}\right)\right\}+\varepsilon .
$$

Assume furthermore that $\left\{\boldsymbol{u}^{\varepsilon}\right\}_{\varepsilon>0}$ is weakly compact in $H^{1, p}$ and let $\boldsymbol{u}^{\varepsilon_{n}} \rightarrow \boldsymbol{u}$ be a weakly convergent subsequence with limit $u$ as $\varepsilon_{n} \rightarrow 0$. Then

$$
\begin{gathered}
I(u)+g(u) \leqq I(v)+g(v) \quad \forall v \in H^{1, p}\left(\Omega ; \mathbb{R}^{N}\right), \\
\min \left\{I(u)+g(u) \mid u \in H^{1, p}\left(\Omega ; \mathbb{R}^{N}\right)\right\}=\lim _{\varepsilon \rightarrow 0} \inf \left\{I^{\varepsilon}(u)+g(u) \mid u \in H^{1, p}\left(\Omega ; \mathbb{R}^{N}\right)\right\} .
\end{gathered}
$$

The homogenization result for (2.4) becomes (cf. [Mü 87, Thm. 1.3, Cor. 2.3]):

Theorem 2.3. Assume that $\Omega$ is a bounded and Lipschitz domain and that (2.1)-(2.3) hold. Then the $I^{\varepsilon}$ as given by (2.4) are $\Gamma$-convergent to I given by 
(2.5), and

$$
\bar{W}(F)=\inf _{k \in \mathbb{N}} \hat{W}^{k}(F),
$$

where

$$
\hat{W}^{k}(F)=\inf \left\{\frac{1}{k^{N}} \int_{k Y} W(x, F+\nabla \boldsymbol{q}) d x \mid \boldsymbol{q} \in H_{k \neq}^{1, p}\right\} .
$$

MARCELLINI [Ma 78] has proved that if $F \mapsto W(x, F)$ is convex the expression for $\bar{W}$ simplifies to $\bar{W}=\hat{W}=\hat{W}^{1}$, while [Mü 87, Thm. 4.3] gives an example where $\bar{W}<\hat{W}$. It seems that not much is known about specific properties of $\bar{W}$; see PonTe-Casteneda [PC 89] for some non-trivial bounds on $\bar{W}$.

We remark that Theorem 2.3 does not require any convexity assumption on $W$. In fact, by an abstract result from the theory of $\Gamma$-convergence (see, e.g., [A 84, Chapter 2.1]) the $\Gamma$-limit remains unchanged if we replace $I^{\varepsilon}$ by its lower semicontinuous envelope with respect to weak convergence of sequences in $H^{1, p}$, which amounts to replacing $W(x, \cdot)$ by its quasiconvex envelope $Q W(x, \cdot)$ (see ACERBI \& FUSCO [AF 84]). Here the quasiconvex envelope $Q f$ of a given function $f$ is the supremum over all quasiconvex functions less than or equal to $f$ (cf. MoRREY [M 52, M 66]). A function $g: \mathbb{R}^{N \times N} \rightarrow \mathbb{R}$ is said to be quasiconvex if for all $N \times N$ matrices $F$, all open sets $\Omega$ and all $\phi \in H^{1, \infty}\left(\Omega ; \mathbb{R}^{N}\right)$,

$$
\int_{\Omega} g(F+\nabla \varphi) d x \geqq \int_{\Omega} g(F) d x .
$$

An example of a quasiconvex but not convex function is given by $F \mapsto \operatorname{det} F$.

If $g$ is quasiconvex, it is rank-1 convex, i.e., for all $N \times N$ matrices $F$ and rank-1 matrices $G=\boldsymbol{a} \otimes \boldsymbol{b}$ with $\boldsymbol{a}, \boldsymbol{b} \in \mathbb{R}^{N}$ the function $t \mapsto g(F+t G)$ is convex. Whether or not rank-1 convexity in turn implies quasiconvexity has been a major open problem in the vector-valued calculus of variations. Very recently ŠVERÁK [Sv 92] has found a striking counterexample showing that for $N \geqq 3$ rank-1 convexity does not imply quasiconvexity. Assuming adequate differentiability of $g$, rank-one convexity is equivalent to

$$
\frac{\partial^{2} g}{\partial F^{2}}(G, G) \geqq 0,
$$

for all $G=\boldsymbol{a} \otimes \boldsymbol{b}$. In the present work we always assume that $W(x, \cdot)$ is quasiconvex and strongly elliptic, i.e.,

$$
\frac{\partial^{2} W}{\partial F^{2}}(x, F)(\boldsymbol{a} \otimes \boldsymbol{b}, \boldsymbol{a} \otimes \boldsymbol{b}) \geqq c_{0}|\boldsymbol{a} \otimes \boldsymbol{b}|^{2}, \quad c_{0}>0,
$$

since we want to study possible instabilities (such as buckling), due to the way in which the different base materials of the composite are mixed, rather than instabilities which would already occur in a homogeneous block made of one of the base materials.

Again by abstract results on $\Gamma$-convergence (cf., e.g., Аттоuch [A 84], Chapter 2.1) the limit functional $I$ is always weakly lower semicontinuous so that $\bar{W}$ is always quasiconvex, and hence rank-1 convex. We are interested in 
whether or not $\bar{W}$ is strictly rank-1 convex (for a twice differentiable $\bar{W}$, whether or not $\bar{W}$ is strongly elliptic).

Failure of strict rank-1 convexity (or strong ellipticity) in the homogenized energy density $\bar{W}$ indicates the possibility of instabilities for the homogenized material. The connection between internal buckling in the composite and the loss of strict rank-1 convexity of $\bar{W}$ will be discussed in Section 5 .

\section{The Linearized System (1): Results}

\subsection{Convergence of solutions for $\Lambda>0$}

Let $\Omega$ be a bounded Lipschitz domain of $\mathbb{R}^{N}$ and let be $v_{0} \in H^{1}\left(\Omega ; \mathbb{R}^{N}\right)$, $f \in H^{-1}\left(\Omega ; \mathbb{R}^{N}\right)$. In this section we study the linearized system

$$
\begin{aligned}
-\operatorname{div}\left(\amalg\left(\frac{x}{\varepsilon}\right) \nabla \boldsymbol{v}^{\varepsilon}\right) & =f \quad \text { in } \Omega, \\
\boldsymbol{v}^{\varepsilon} & =v_{0} \quad \text { on } \partial \Omega .
\end{aligned}
$$

We assume that the real 4-tensor $\mathbb{L}$ is defined on all $\mathbb{R}^{N}$, is measurable and a.e. in $\mathbb{R}^{N}$ satisfies

$$
\begin{gathered}
\qquad(x+z)=\Perp(x) \quad \text { for all } z \in \mathbb{Z}^{N}, \\
(\amalg(x) A, B)=(A, \Perp(x) B) \quad \text { for all real } N \times N \text { matrices } A, B, \\
|\amalg(x) A| \leqq C|A| \quad \text { for all real } N \times N \text { matrices } A,
\end{gathered}
$$$$
(\mathbb{L}(x) \boldsymbol{a} \otimes \boldsymbol{b}, \boldsymbol{a} \otimes \boldsymbol{b}) \geqq c|\boldsymbol{a} \otimes \boldsymbol{b}|^{2}=c|\boldsymbol{a}|^{2}|\boldsymbol{b}|^{2}, \quad c>0 \quad \text { for all } \boldsymbol{a}, \boldsymbol{b} \in \mathbb{R}^{N} \text {. }
$$

The best ellipticity constant for the nonhomogeneous tensor $\Perp(x)$ is

$$
\alpha_{\Downarrow}=\operatorname{ess} \inf _{x \in Y} \min _{\substack{|\boldsymbol{a}|=|\boldsymbol{b}|=1 \\ \boldsymbol{a}, \boldsymbol{b} \in \mathbb{R}^{N}}}(\mathbb{L}(x) \boldsymbol{a} \otimes \boldsymbol{b}, \boldsymbol{a} \otimes \boldsymbol{b}),
$$

and from the previous assumptions it follows that $\alpha_{\mathbb{L}}>0$.

The previous assumptions imply that the bilinear symmetric form

$$
a^{\varepsilon}(\boldsymbol{u}, \boldsymbol{v})=\int_{\Omega}\left(\left\lfloor\left(\frac{x}{\varepsilon}\right) \nabla \boldsymbol{u}, \nabla \boldsymbol{v}\right) d x\right.
$$

is continuous on $H^{1}\left(\Omega ; \mathbb{R}^{N}\right)$. A weak solution $v^{\varepsilon} \in H^{1}\left(\Omega ; \mathbb{R}^{N}\right)$ of (3.1), (3.2) is a solution of the following problem where $\langle$,$\rangle denotes the duality pairing$ between $H^{-1}\left(\Omega ; \mathbb{R}^{N}\right)$ and $H_{0}^{1}\left(\Omega ; \mathbb{R}^{N}\right)$.

$\left(\mathrm{P}^{\varepsilon}\right)$ Find $\boldsymbol{v}^{\varepsilon} \in H^{1}\left(\Omega ; \mathbb{R}^{N}\right)$ such that $\boldsymbol{v}^{\varepsilon}-\boldsymbol{v}_{0} \in H_{0}^{1}\left(\Omega ; \mathbb{R}^{N}\right)$ and such that

$$
a^{\varepsilon}\left(\boldsymbol{v}^{\varepsilon}, \varphi\right)=\langle f, \varphi\rangle \text { for all } \varphi \in H_{0}^{1}\left(\Omega ; \mathbb{R}^{N}\right) \text {. }
$$


Since $a^{\varepsilon}$ is a symmetric, (3.8) is the weak form of the Euler-Lagrange equation corresponding to a critical point of the functional

$$
I^{\varepsilon}(u)=\frac{1}{2} a^{\varepsilon}(u, u)-\left\langle f, u-v_{0}\right\rangle
$$

on the affine set

$$
\mathbb{K}_{v_{0}}=\left\{u \in H^{1}\left(\Omega ; \mathbb{R}^{N}\right) ; \boldsymbol{u}-\boldsymbol{v}_{0} \in H_{0}^{1}\left(\Omega ; \mathbb{R}^{N}\right)\right\} .
$$

Thus a weak solution of (3.1), (3.2) is also a solution of

$\left(\mathrm{Q}^{\varepsilon}\right)$ Find $\boldsymbol{v}^{\varepsilon} \in \mathbb{K}_{v_{0}}$ such that for all $\boldsymbol{u} \in \mathbb{K}_{v_{0}}$,

$$
I^{\varepsilon}\left(v^{\varepsilon}\right) \leqq I^{\varepsilon}(u) .
$$

We are interested in the behavior of the solutions $v^{\varepsilon}$ of the problem $\left(\mathrm{P}^{\varepsilon}\right)$ or $\left(\mathrm{Q}^{\varepsilon}\right)$ as $\varepsilon \rightarrow 0$. If $u$ is a scalar, (3.6) implies that the bilinear form $a^{\varepsilon}(u, v)$ is uniformly coercive (with respect to $\varepsilon>0$ ) on $H_{0}^{1}(\Omega)$ and so the existence and the uniqueness of $v^{\varepsilon}$ and the boundedness in $H^{1}(\Omega)$ of the sequence $v^{\varepsilon}$ follow.

The situation is completely different when $\boldsymbol{u}$ is a vector. Indeed, even when the coefficients of the 4-tensor $\mathbb{L}$ are continuous, the strong ellipticity condition (3.6) only implies Garding's inequality, i.e., that there exist $c_{1}>0$ and $\delta(\varepsilon)>0$ such that for all $\boldsymbol{u} \in H_{0}^{1}\left(\Omega ; \mathbb{R}^{N}\right)$,

$$
a^{\varepsilon}(\boldsymbol{u}, \boldsymbol{u}) \geqq c_{1}\|\boldsymbol{u}\|_{H_{0}^{1}}^{2}-\delta(\varepsilon)\|\boldsymbol{u}\|_{L^{2}}^{2} .
$$

This inequality does not imply uniform coercivity, as shown by LEDRET [LD 87]. In order to measure the coercivity of $\mathbb{L}$ we introduce the quantity

where

$$
\Lambda=\inf \left\{Q\left(\nabla \boldsymbol{v} ; \mathbb{R}^{N}\right) \mid \boldsymbol{v} \in \mathscr{D}\left(\mathbb{R}^{N}, \mathbb{R}^{N}\right)\right\},
$$

$$
Q(w ; \mathscr{B})=\frac{\int_{\mathscr{B}}(\Perp(x) w, w) d x}{\int_{\mathscr{A}}(w, w) d x} .
$$

We can now state our first result in terms of the constant $\Lambda$.

Theorem 3.1. Assume that (3.3)-(3.6) hold and that $\Lambda>0$.

(i) For every $\varepsilon>0$ the system (3.1), (3.2) has a unique solution $v^{\varepsilon}$ and there exists $C>0$ such that for all $\varepsilon>0$,

$$
\left\|v^{\varepsilon}\right\|_{H^{1}} \leqq C\left(\|f\|_{H^{-1}}+\left\|v_{0}\right\|_{H^{\mathrm{k}}}\right),
$$

and $v^{\varepsilon} \rightarrow v$ weakly in $H^{1}\left(\Omega ; \mathbb{R}^{N}\right)$ for $\varepsilon \rightarrow 0$.

(ii) $v$ is the unique solution of

$$
\begin{gathered}
-\operatorname{div}(M \nabla v)=f \quad \text { in } \Omega, \\
v=v_{0} \quad \text { on } \partial \Omega,
\end{gathered}
$$

where the constant real symmetric 4-tensor $\mathbb{M}$ can be characterized by

$$
\mathbb{M} F=\int_{Y} \Perp(x)(F+\nabla \tau) d x,
$$


where $\tau \in H_{\#}^{1}$ satisfies

$$
\int_{Y}(\mathbb{L}(x)(F+\nabla \tau), \nabla z) d x=0 \quad \text { for all } z \in H_{\#}^{1} .
$$

Equivalently, $\mathbb{M}$ is characterized by

$$
(M F, F)=\inf \left\{\int_{Y}(\mathbb{L}(x)(F+\nabla \boldsymbol{q}), F+\nabla \boldsymbol{q}) d x \mid \boldsymbol{q} \in H_{\#}^{1}\right\} .
$$

Moreover, for all $\boldsymbol{a}, \boldsymbol{b} \in \mathbb{R}^{N}$,

$$
(\mathbb{M} a \otimes b, a \otimes b) \geqq \Lambda|a \otimes b|^{2}=\Lambda|a|^{2}|b|^{2} .
$$

(iii) The same conclusions hold if the prescription of $v^{0}$ and $f$ is replaced by the prescription of sequences $v_{\varepsilon}^{0}$ and $f_{\varepsilon}$ with $v_{\varepsilon}^{0} \rightarrow v^{0}$ strongly in $H^{1}\left(\Omega ; \mathbb{R}^{N}\right)$ and $f^{\varepsilon} \rightarrow f$ strongly in $H^{-1}\left(\Omega ; \mathbb{R}^{N}\right)$.

For $A<0$ the problem $\left(\mathrm{Q}^{\varepsilon}\right)$ has no solution, since we have the following result.

Proposition 3.2. Assume that $A<0$. Then, for $0<\varepsilon<\varepsilon_{0}$ with $\varepsilon_{0}$ sufficiently small, there exists $\boldsymbol{w}^{\varepsilon} \in H_{0}^{1}\left(\Omega ; \mathbb{R}^{N}\right)$ such that $\left\|\nabla \boldsymbol{w}^{\varepsilon}\right\|_{L^{2}}=1$ and

$$
a^{\varepsilon}\left(w^{\varepsilon}, w^{\varepsilon}\right) \leqq \frac{1}{2} \Lambda<0 .
$$

Consequently $I^{\varepsilon}$ is not bounded from below on $\mathbb{K}_{v_{0}}$.

Proof. It follows from the definition of $\Lambda$ that there is a $v \in \mathscr{D}\left(\mathbb{R}^{N} ; \mathbb{R}^{N}\right)$ such that $\|\nabla \boldsymbol{v}\|_{L^{2}}=1$ and

$$
\int_{\mathbb{R}^{N}}(\mathbb{L}(\mathrm{x}) \nabla \boldsymbol{v}, \nabla \boldsymbol{v}) d x \leqq \frac{1}{2} \Lambda .
$$

Let $K$ denote the support of $v$. For all sufficiently small $\varepsilon>0$ there exists $z_{\varepsilon} \in \mathbb{Z}^{N}$ such that $\varepsilon\left(-z_{\varepsilon}+K\right) \subset \Omega$. Let

$$
\boldsymbol{w}^{\varepsilon}(x)=\varepsilon^{-(N-2) / 2} v\left(\frac{x}{\varepsilon}+z_{\varepsilon}\right) .
$$

Then $\boldsymbol{w}^{\varepsilon} \in H_{0}^{1}\left(\Omega ; \mathbb{R}^{N}\right),\left\|\nabla \boldsymbol{w}^{\varepsilon}\right\|_{L^{2}}=1$ and, by the periodicity of $\llbracket$,

$$
a^{\varepsilon}\left(\boldsymbol{w}^{\varepsilon}, \boldsymbol{w}^{\varepsilon}\right)=\int_{\Omega}\left(\Perp\left(\frac{x}{\varepsilon}\right) \nabla \boldsymbol{w}^{\varepsilon}, \nabla \boldsymbol{w}^{\varepsilon}\right) d x=\int_{\mathbb{R}^{N}}(\Perp(x) \nabla \boldsymbol{v}, \nabla \boldsymbol{v}) d x \leqq \frac{1}{2} \Lambda .
$$

To show that $I^{\varepsilon}$ is not bounded from below on $\mathbb{K}_{\boldsymbol{v}_{0}}$ consider $I^{\varepsilon}\left(v_{0}+t w^{\varepsilon}\right)$ and let $t \rightarrow+\infty$. 


\section{2. $\Gamma$-convergence for $\Lambda \geqq 0$}

For $A=0$ the following quantities which measure the coercivity of $\mathbb{L}$ are needed:

$$
\begin{aligned}
& \Lambda_{4}=\inf \left\{Q(\boldsymbol{a} \otimes \boldsymbol{b}+\nabla \boldsymbol{q} ; Y) \mid \boldsymbol{a} \in \mathbb{C}^{N}, \boldsymbol{b} \in \mathbb{R}^{N}, \boldsymbol{q} \in \mathscr{H}_{\#}^{1}\right\}, \\
& \Lambda_{5}=\liminf _{\omega \rightarrow 0} \inf \left\{Q\left(\nabla\left(e^{i \omega x} \boldsymbol{q}\right) ; Y\right) \mid \boldsymbol{q} \in \mathscr{H}_{\#}^{1}\right\}, \\
& \Lambda_{6}=\inf \left\{Q(\nabla \boldsymbol{q} ; \nabla) \mid \boldsymbol{q} \in \mathscr{H}_{\sharp}^{1}\right\} .
\end{aligned}
$$

Here we have extended $Q$ to complex-valued functions due to requirements of the subsequent discussion. Note that if $X$ is a subsequence of $H^{1}\left(\mathscr{B} ; \mathbb{R}^{N}\right)$ and $X \oplus i X$ its complexification, we have

$$
\inf \{Q(\nabla v ; \mathscr{B}) \mid v \in X \oplus i X\}=\inf \{Q(\nabla v ; \mathscr{B}) \mid v \in X\} .
$$

Indeed simply write $v=\operatorname{Re} v+i \operatorname{Re}(-i v)=w_{1}+i w_{2}$. Then, by symmetry of $\mathbb{L}$,

$$
\int_{\mathscr{S}}(\mathbb{H}(x) \nabla \boldsymbol{v}, \nabla \boldsymbol{v}) d x=\int_{\mathscr{\mathscr { B }}}\left(\mathbb{L}(x) \nabla \boldsymbol{w}_{1}, \nabla \boldsymbol{w}_{1}\right) d x+\int_{\mathscr{\mathscr { B }}}\left(\mathbb{L}(x) \nabla \boldsymbol{w}_{2}, \nabla \boldsymbol{w}_{2}\right) d x
$$

and hence

$$
Q(\nabla \boldsymbol{v} ; \mathscr{B}) \geqq \min \left\{Q\left(\nabla \boldsymbol{w}_{1} ; \mathscr{B}\right), Q\left(\nabla \boldsymbol{w}_{2} ; \mathscr{B}\right)\right\} .
$$

The interest of the previous quantities rests on the following result. Recall that $\alpha_{\Perp}$ is the best ellipticity constant of $\Perp(x)$ given by (3.7).

Theorem 3.3. Assume (3.3)-(3.6) hold and so $\alpha_{\Perp}>0$ (see (3.7)). Then (i) $\Lambda \leqq \Lambda_{4}=\Lambda_{5} \leqq \Lambda_{6} \leqq \alpha_{\Perp}$.

(ii) If $\Lambda \geqq 0$, then for all $k=1,2,3, \ldots$ and every $N \times N$ matrix $F$ the functionals

$$
\boldsymbol{q} \rightarrow \mathscr{L}_{k}(\boldsymbol{q} ; F)=\frac{1}{k^{N}} \int_{k Y}(\Perp(x)(F+\nabla \boldsymbol{q}), F+\nabla \boldsymbol{q}) d x
$$

are convex and weakly sequentially lower semicontinuous (wslsc) on $\mathscr{H}_{k \sharp}^{1}$. (iii) If $\Lambda=\Lambda_{6}=0$, define

$$
N_{k}=\left\{q \in \mathscr{H}_{k \#}^{1} \mid \int_{k Y}(\mathbb{L}(x) \nabla \boldsymbol{q}, \nabla \boldsymbol{q}) d x=0, \int_{k Y} \boldsymbol{q} d x=0\right\} .
$$

Then $N_{k}$ is a closed subspace of $\mathscr{H}_{k^{4}}^{1}$. Its orthogonal complement is denoted by $N_{k}^{\perp}$.

(iv) If $\Lambda \geqq 0$, define for all $k=2,3, \ldots$ a symmetric 4-tensor $M_{k}$ by

$$
\left(\mathbb{M}_{k} F, F\right)=\inf \left\{\mathscr{L}_{k}(\boldsymbol{q} ; F) \mid \boldsymbol{q} \in \mathscr{H}_{k \#}^{1}\right\} .
$$

Then for all matrices $F$,

$$
\left(\mathbb{M}_{k} F, F\right)=(\mathbb{M} F, F),
$$

where the 4-tensor $\mathbb{M}$ is defined by (3.18). 
(v) For $\Lambda_{4} \geqq 0$ the 4-tensor $M$ defined by (3.18) satisfies

$(\mathbb{M} \boldsymbol{a} \otimes \boldsymbol{b}, \boldsymbol{a} \otimes \boldsymbol{b})=\inf \left\{\mathscr{L}_{1}(\boldsymbol{q} ; \boldsymbol{a} \otimes \boldsymbol{b}) \mid \boldsymbol{q} \in \mathscr{H}_{\#}^{1}\right\} \geqq \Lambda_{4}|\boldsymbol{a} \otimes \boldsymbol{b}|^{2} \quad$ for all $\boldsymbol{a}, \boldsymbol{b} \in \mathbb{R}^{N}$.

(vi) If $\Lambda_{6}>0$, then $\mathscr{L}_{1}$ attains its minimum on $\mathscr{H}^{1}$ for every $N \times N$ matrix $F$. (vii) If $\Lambda_{6} \geqq 0$, then for every $\omega \in \mathbb{R}^{N}$ the functionals

$$
\boldsymbol{q} \rightarrow \hat{\mathscr{L}}_{1}(\boldsymbol{q} ; \omega)=\int_{Y}\left(\amalg(x) \nabla\left(e^{i \omega \cdot x} q\right), \nabla\left(e^{i \omega \cdot x} q\right)\right) d x
$$

are convex and weakly sequentially lower semicontinuous on $\mathscr{H} \stackrel{1}{\#}$.

We can now state the result in the case $A=0$.

Theorem 3.4. Let (3.3)-(3.6) hold and assume that $\Lambda \geqq 0, \Lambda_{6}>0$. Then (i) The functionals $a^{\varepsilon}(\boldsymbol{u}, \boldsymbol{u})$ on $\mathbb{K}_{\boldsymbol{v}_{0}}$ are $\Gamma$-convergent (with respect to the weak convergence of $\left.H^{1}\left(\Omega ; \mathbb{R}^{N}\right)\right)$ to the functional

$$
a(\boldsymbol{u}, \boldsymbol{u})=\int_{\Omega}(\mathbb{M} \nabla \boldsymbol{u}, \nabla \boldsymbol{u}) d x
$$

where $M$ is the 4-tensor defined by (3.18).

(ii) If $\Lambda=0$ and $\Lambda_{4}>0$, then $M$ remains strictly strongly elliptic, i.e.,

$$
(\mathbb{M} \boldsymbol{a} \otimes \boldsymbol{b}, \boldsymbol{a} \otimes \boldsymbol{b}) \geqq \Lambda_{4}|\boldsymbol{a} \otimes \boldsymbol{b}|^{2}
$$

for all $\boldsymbol{a}, \boldsymbol{b} \in \mathbb{R}^{N}$, the infimum in (3.18) is attained and there exists $\omega \neq 0$ $\left(\bmod (2 \pi \mathbb{Z})^{N}\right)$ and $\boldsymbol{q} \in H_{\#}^{1}$ with $\nabla \boldsymbol{q} \equiv 0$ such that

$$
-\operatorname{div}\left(\Perp(x) \nabla\left(e^{i \omega \cdot x} \boldsymbol{q}\right)\right)=0 \quad \text { on } \mathbb{R}^{N} .
$$

(iii) If $\Lambda=\Lambda_{4}=0$, then there exists $a, b \in \mathbb{R}^{N} \backslash\{0\}$ and $q \in H_{\#}^{1}$ such that

$$
-\operatorname{div}(\Perp(x)(\boldsymbol{a} \otimes \boldsymbol{b}+\nabla \boldsymbol{q}))=0 \quad \text { on } \mathbb{R}^{N} .
$$

Moreover, for that choice of $a, b$,

$$
(\mathbb{M} \boldsymbol{a} \otimes \boldsymbol{b}, \boldsymbol{a} \otimes \boldsymbol{b})=0,
$$

i.e., the homogenized 4-tensor $\mathbb{M}$ loses strict strong ellipticity.

If the coercivity of the homogenized tensor $M$ is measured by its best ellipticity constant

$$
\alpha_{M}=\min _{\substack{|a|=|b|=1 \\ a, b \in \mathbb{R}^{N}}}(\mathbb{M} \boldsymbol{a} \otimes b, \boldsymbol{a} \otimes \boldsymbol{b}),
$$

then an immediate consequence of Theorem 3.4 is

Corollary 3.5. Let $\Lambda \geqq 0$. Then $\alpha_{\mathbb{M}} \geqq \Lambda_{4}$ and $\alpha_{M}=0$ if $\Lambda_{4}=0$.

Remark. The functions $e^{i \omega \cdot x} q$ in (3.26) are sometimes referred to as Bloch waves, while those in (3.27) may be associated with shearing deformations (modulated by a periodic contribution). Failure of ellipticity for the homoge- 
nized material is thus related to the existence of these shearing deformations or equivalently to long-wavelength ( $\omega \rightarrow 0$ ) solutions (cf. the definition of $\Lambda_{5}$ and the fact that $\Lambda_{5}=\Lambda_{4}$ ).

The study of the case $A=\Lambda_{6}=0$ is less complete. Recall the definition of $N_{1}^{\perp}$ from Theorem 3.3 (iii).

Proposition 3.6. Let (3.3)-(3.6) hold and assume $\Lambda=\Lambda_{6}=0$. Define

$$
\begin{aligned}
& \tilde{\Lambda}_{4}=\inf \left\{Q(\boldsymbol{a} \otimes \boldsymbol{b}+\nabla \boldsymbol{q}, Y) \mid \boldsymbol{a} \in \mathbb{C}^{N}, \boldsymbol{b} \in \mathbb{R}^{N}, \boldsymbol{q} \in N_{1}^{\perp}\right\}, \\
& \tilde{\Lambda}_{5}=\liminf _{\omega \rightarrow 0} \inf \left\{Q\left(\nabla\left(e^{i \omega \cdot x} \boldsymbol{q}\right), Y\right) \mid \boldsymbol{q} \in N_{1}^{\perp}\right\} .
\end{aligned}
$$

Then $\tilde{\Lambda}_{4}=\tilde{\Lambda}_{5} \geqq 0$ and the 4-tensor $M$ defined by (3.18) satisfies

$$
(\mathbb{M} \boldsymbol{a} \otimes \boldsymbol{b}, \boldsymbol{a} \otimes \boldsymbol{b}) \geqq \tilde{\Lambda}_{4}|\boldsymbol{a} \otimes \boldsymbol{b}|^{2} .
$$

If $\tilde{\Lambda}_{4}=0$, there exist $\boldsymbol{a}, \boldsymbol{b} \in \mathbb{R}^{N} \backslash\{0\}$ such that

$$
(M a \otimes b, a \otimes b)=0 .
$$

\subsection{Summary of the coercivity constants}

To clarify the meaning of the various coercivity constants, let us briefly summarize their relevance and their mutual relationship.

$\Lambda \quad$ measures the global coercivity of the nonhomogeneous tensor $\mathbb{L}(x)$. It can be computed by using smooth functions (see (3.11)) or equivalently by using Bloch waves (see Lemma 4.2 below).

$\Lambda_{4}=\Lambda_{5}$ measures coercivity with respect to long-wavelength $(\omega \rightarrow 0)$ perturbations or, equivalently, with respect to shearing deformations (both modulo $Y$-periodic contributions).

$\Lambda_{6} \quad$ measures coercivity with respect to $Y$-periodic, possibly highly localized deformations.

$\alpha_{\Perp} \quad$ is the best ellipticity constant for $\mathbb{L}$.

One has (assuming as always $\Lambda \geqq 0$ ):

Finally

$$
\Lambda \leqq \Lambda_{4}=\Lambda_{5} \leqq \Lambda_{6} \leqq \alpha_{\llbracket}
$$

$\alpha_{\mathbb{M}}$ measures the coercivity of the homogenized tensor $M$ and satisfies $\alpha_{\mathbb{M}} \geqq \Lambda_{4}$ and $\alpha_{\mathbb{M}}=0$ if $\Lambda_{4}=0$.

\subsection{Layered materials}

In Section 6, we consider a layered (or stratified) material, i.e., a material whose elastic properies only change in one coordinate direction. Here we briefly indicate how the theory developed above simplifies in that special case. 
Assuming that the elasticity tensor $\Perp(x)$ is independent of $x_{1}, \ldots, x_{N-1}$ and periodic with period 1 in $x_{N}$ we define the following quantities in analogy with (3.18), (3.20)-(3.22), (3.29) and (4.4). We do not distinguish between a function on $\mathbb{R}$ and its extension to $\mathbb{R}^{N}$ as a function of the last variable only.

$$
\begin{aligned}
& \Lambda_{1}^{s}=\inf \left\{Q(\nabla \boldsymbol{v} ; Y) \mid \boldsymbol{v}=e^{i \omega \cdot x} \boldsymbol{p}\left(x_{N}\right), \omega \in \mathbb{R}^{N-1} \times\left[0,2 \pi\left[, \boldsymbol{p} \in \mathscr{H}_{\#}^{1}([0 ; 1])\right\},\right.\right. \\
& \Lambda_{4}^{s}=\inf \left\{Q(\boldsymbol{a} \otimes \boldsymbol{b}+\nabla \boldsymbol{q} ; Y) \mid \boldsymbol{a} \in \mathbb{C}^{N}, \boldsymbol{b} \in \mathbb{R}^{N}, \boldsymbol{q} \in \mathscr{H}_{\#}^{1}([0 ; 1])\right\}, \\
& \Lambda_{5}^{s}=\liminf _{\omega \rightarrow 0} \inf \left\{Q\left(\nabla\left(e^{i \omega \cdot x} q\right) ; Y\right) \mid q \in \mathscr{H}_{\#}^{1}([0 ; 1])\right\}, \\
& \Lambda_{6}^{s}=\inf \left\{Q(\nabla \boldsymbol{q} ; Y) \mid \boldsymbol{q} \in \mathscr{H}_{\#}^{1}([0 ; 1])\right\}, \\
& \left(M^{s} G, G\right)=\inf _{\psi \in H_{H}([0,1])} \int_{0}^{1}\left(\amalg\left(x_{N}\right)(G+\nabla \psi), G+\nabla \psi\right) d x_{N}, \\
& \alpha_{M}^{s}=\min _{\substack{|\boldsymbol{a}|=|\boldsymbol{b}|=1 \\
\boldsymbol{a}, \boldsymbol{b} \in \mathbb{R}^{N}}}\left(\mathbb{M}^{s} \boldsymbol{a} \otimes \boldsymbol{b}, \boldsymbol{a} \otimes \boldsymbol{b}\right) .
\end{aligned}
$$

A stratified material may be viewed as a material having a periodic cell $Z=\left[0, H_{1}\right] \times \ldots\left[0, H_{N-1}\right] \times[0,1]$, for any choice of $H_{i}>0$. The homogenized tensor should thus be given by

$$
\left(M_{H} G, G\right)=\inf _{\psi \in H_{*}^{\prime}(Z)} \frac{1}{\text { meas } Z} \int_{Z}(\mathbb{L}(x)(G+\nabla \psi), G+\nabla \psi) d x .
$$

The results for stratified materials can be summarized in

Theorem 3.7. Let (3.3) to (3.6) hold and assume $\Lambda_{1}^{s} \geqq 0$. Then

(i) $\Lambda=\Lambda_{1}^{s}$.

(ii) $\Lambda_{1}^{s} \leqq \Lambda_{4}^{s}=\Lambda_{5}^{s} \leqq \Lambda_{6}^{s}$.

(iii) If $\Lambda_{1}^{s}>0$, then the unique solutions $v^{\varepsilon}$ of (3.1), (3.2) converge weakly in $H^{1}$ to the unique solution of

$$
\begin{aligned}
-\operatorname{div} \mathbb{M}^{s} \nabla v & =f & & \text { in } \Omega, \\
v & =v_{0} & & \text { on } \partial \Omega .
\end{aligned}
$$

(iv) The functionals $a^{\varepsilon}(\boldsymbol{u}, \boldsymbol{u})$ on $\mathbb{K}_{\boldsymbol{v}_{0}}$ are $\Gamma$-convergent to the functional

$$
\boldsymbol{a}(\boldsymbol{u}, \boldsymbol{u})=\int_{\Omega}\left(\mathbb{M}^{s} \nabla \boldsymbol{u}, \nabla \boldsymbol{u}\right) d x .
$$

In particular,

$$
\mathbb{M}^{s}=\mathbb{M}_{H}
$$

for all choices of $H=\left(H_{1}, \ldots, H_{N-1}\right)$ (with $H_{i}>0$ ) and moreover

$$
\left(\mathbb{M}^{s} \boldsymbol{a} \otimes \boldsymbol{b}, \boldsymbol{a} \otimes \boldsymbol{b}\right) \geqq \Lambda_{4}^{s}|\boldsymbol{a} \otimes \boldsymbol{b}|^{2},
$$

so that

$$
\alpha_{\mathbb{M}}^{s} \geqq \Lambda_{4}^{s} .
$$


(v) If $\Lambda_{1}^{s}=0$ and $\Lambda_{4}^{s}>0$, then there exists a Bloch-wave solution, i.e., there are $\omega \in \mathbb{R}^{N}$, with $\left(\omega_{1}, \ldots, \omega_{N-1}\right) \neq 0$ or $\omega_{N} \neq 0 \quad(\bmod 2 \pi)$, and $q \in H_{\#}^{1}([0,1]), \nabla q \equiv 0$ such that

$$
-\operatorname{div} \mathbb{L}(x) \nabla\left(e^{i \omega \cdot x} q\right)=0 \quad \text { on } \mathbb{R}^{N} .
$$

(vi) If $\Lambda_{1}^{s}=\Lambda_{4}^{s}=0$ and if $\Lambda_{6}^{s}>0$, there exists a long-wavelength solution, i.e., there are $\boldsymbol{a}, \boldsymbol{b} \in \mathbb{R}^{N} \backslash\{0\}$ and $\boldsymbol{q} \in H_{\#}^{1}([0,1]), \nabla \boldsymbol{q} \equiv 0$ such that

$$
-\operatorname{div} \mathbb{L}(x)(\boldsymbol{a} \otimes \boldsymbol{b}+\nabla \boldsymbol{q})=0 \quad \text { on } \mathbb{R}^{N} .
$$

Moreover, for this choice of $\boldsymbol{a}$ and $\boldsymbol{b}$

$$
(M a \otimes b, a \otimes b)=0 .
$$

(vii) Define the best stratified ellipticity constant for $\mathbb{L}$ as

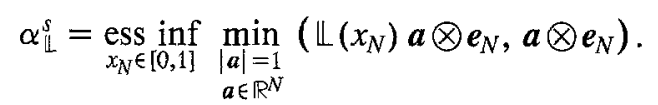

Then

$$
\alpha_{\Perp} \leqq \Lambda_{6}^{s}=\alpha_{\llbracket}^{s} .
$$

Remark. Two cases can occur: either $\Lambda<\Lambda_{6}^{s}$ or $\Lambda=\Lambda_{6}^{s}$. In the latter case, $\Lambda=\alpha_{\mathbb{1}}=\Lambda_{6}^{s}=\alpha_{\Perp}^{s}$.

\section{The Linearized System (II): Proofs}

\subsection{Preliminary results}

We begin with some preliminary results.

For $\omega \in \mathbb{R}^{N}$ let us define

$$
\begin{gathered}
C(\omega)=\inf \left\{\frac{|\omega+2 \pi z|^{2}}{1+|2 \pi z|^{2}} ; z \in \mathbb{Z}^{N}\right\}, \\
C^{*}(\omega)=\inf \left\{\frac{|\omega+2 \pi z|^{2}}{1+|2 \pi z|^{2}} ; z \in \mathbb{Z}^{N} \backslash\{0\}\right\} .
\end{gathered}
$$

Recall that $\mathscr{H}_{\#}^{1}$ denotes the space of $\mathbb{C}^{N}$-valued $H^{1}$ functions periodic on the unit cell $Y=[0,1]^{N}$

Lemma 4.1. (i) For every $q \in \mathscr{H} \frac{1}{\#}$ and every $\omega \in \mathbb{R}^{N}$,

$$
C(\omega) \int_{Y}\left\{|\boldsymbol{q}|^{2}+|\nabla \boldsymbol{q}|^{2}\right\} d x \leqq \int_{Y}|\boldsymbol{q} \otimes i \omega+\nabla \boldsymbol{q}|^{2} d x=\int_{Y}\left|\nabla\left(e^{i \omega \cdot x} \boldsymbol{q}\right)\right|^{2} d x .
$$

(ii) If, moreover, $\int_{Y} q d x=0$, then (4.3) holds with $C^{*}(\omega)$ instead of $C(\omega)$.

Proof. It suffices to prove (4.3) for finite sums

$$
\boldsymbol{q}(x)=\sum_{z \in \mathbb{Z}^{N}} e^{2 \pi i z \cdot x} \boldsymbol{a}_{z}
$$


since these $q$ are dense in $\mathscr{H}_{\#}^{1}$. Now

$$
\begin{aligned}
\int_{Y}|\boldsymbol{q} \otimes i \omega+\nabla \boldsymbol{q}|^{2} d x & =\int_{Y}\left|\nabla\left(e^{i \omega \cdot x} \boldsymbol{q}\right)\right|^{2} d x \\
& =\sum_{z}\left|\boldsymbol{a}_{z}\right|^{2}|\omega+2 \pi z|^{2} \geqq C(\omega) \sum_{z}\left|\boldsymbol{a}_{z}\right|^{2}\left(1+|2 \pi z|^{2}\right) \\
& =C(\omega) \int_{Y}\left\{|\boldsymbol{q}|^{2}+|\nabla \boldsymbol{q}|^{2}\right\} d x
\end{aligned}
$$

and (4.3) follows. If $\int_{Y} q d x=0$, then $a_{0}=0$ which gives the result.

The following characterization of $\Lambda$ as defined in (3.11) will be crucial.

Lemma 4.2. Let (3.3)-(3.6) hold and define

$$
\begin{aligned}
& \Lambda_{1}=\inf \left\{Q(\nabla v ; Y) \mid v=e^{i \omega \cdot x} p, \omega \in\left[0,2 \pi^{N}, p \in \mathscr{H}_{H}^{1}\right\},\right. \\
& \Lambda_{2}=\inf \left\{Q(\nabla v ; k Y) \mid v=e^{i \omega \cdot x} q, \omega \in \mathbb{R}^{N}, q \in \mathscr{H}_{k \#}^{1}, k \in \mathbb{N}^{*}\right\}, \\
& \Lambda_{3}=\inf \left\{Q(\nabla v ; k Y) \mid v \in \mathscr{H}_{k \#}^{1}, k \in \mathbb{N}^{*}\right\} .
\end{aligned}
$$

Then $\Lambda=\Lambda_{1}=\Lambda_{2}=\Lambda_{3}$.

Proof. (a) $A \geqq \Lambda_{1}$. We employ a transformation well known in the theory of Bloch waves (cf. [RS 79], [BLP 78]). For $v \in \mathscr{D}\left(\mathbb{R}^{N} ; \mathbb{C}^{N}\right)$ and $\omega \in Y^{*} \stackrel{\text { def }}{=}$ $\left[0,2 \pi\left[^{N}\right.\right.$ define

$$
\tilde{\boldsymbol{v}}_{\omega}(x)=\sum_{z \in \mathbb{Z}^{N}} e^{-i \omega \cdot z} v(x+z)
$$

Note that for a fixed value of $x$ the sum is finite since $v$ has compact support. Since $\tilde{\boldsymbol{v}}_{\omega}(x+z)=e^{i \omega \cdot z} \tilde{\boldsymbol{v}}_{\omega}(x)$ for $z \in \mathbb{Z}^{N}$, we have

$$
\tilde{v}_{\omega}(x)=e^{i \omega \cdot x} p(x)
$$

with $\boldsymbol{p} \in \mathscr{H}_{\#}^{1}$. We assert that

$$
\begin{aligned}
\int_{\mathbb{R}^{N}}|\nabla \boldsymbol{v}|^{2} d x & =(2 \pi)^{-N} \int_{Y} \int_{Y^{*}}\left|\nabla \tilde{\boldsymbol{v}}_{\omega}\right|^{2} d \omega d x, \\
\int_{\mathbb{R}^{N}}(\mathbb{L}(x) \nabla \boldsymbol{v}, \nabla \boldsymbol{v}) d x & =(2 \pi)^{-N} \int_{Y_{Y^{*}}}\left(\mathbb{L}(x) \nabla \tilde{\boldsymbol{v}}_{\omega}, \nabla \tilde{\boldsymbol{v}}_{\omega}\right) d \omega d x .
\end{aligned}
$$

Clearly (4.9) is a special case of (4.10). To prove the latter identity, set $w=\nabla \boldsymbol{v}$ and observe that $\nabla \tilde{\boldsymbol{v}}_{\omega}=\tilde{\boldsymbol{w}}_{\omega}$. Now $\int_{Y} \int_{Y^{*}}\left(\mathbb{}(x) \tilde{w}_{\omega}, \tilde{w}_{\omega}\right) d \omega d x$

$$
\begin{aligned}
& =\int_{Y} \sum_{z, z^{\prime} \in \mathbb{Z}^{N}} \int_{Y^{*}} e^{i \omega \cdot\left(z^{\prime}-z\right)}\left(\mathbb{L}(x) w(x+z), w\left(x+z^{\prime}\right)\right) d \omega d x \\
& =(2 \pi)^{N} \int_{Y} \sum_{z}(\mathbb{L}(x+z) w(x+z), w(x+z)) d x \\
& =(2 \pi)^{N} \int_{\mathbb{R}^{N}}(\mathbb{w} w, w) d x=(2 \pi)^{N} \int_{\mathbb{R}^{N}}(\mathbb{L}(x) \nabla v, \nabla v) d x,
\end{aligned}
$$

where we used $\int_{Y^{*}} e^{i\left(\omega \cdot\left(z^{\prime}-z\right)\right.} d \omega=(2 \pi)^{N} \delta_{z z^{\prime}}$. 
Let us assume that $\Lambda_{1}>-\infty$; otherwise (a) is obvious. Then by (4.8) and the definition of $\Lambda_{1}$,

$$
\int_{Y}\left(\mathbb{L} \nabla \tilde{\boldsymbol{v}}_{\omega}, \nabla \tilde{\boldsymbol{v}}_{\omega}\right) d x \geqq \Lambda_{1} \int_{Y}\left|\nabla \tilde{\boldsymbol{v}}_{\omega}\right|^{2} d x .
$$

Integrating over $\omega$ and using (4.9), (4.10) we have

$$
\int_{\mathbb{R}^{N}}(\mathbb{L} \nabla \boldsymbol{v}, \nabla \boldsymbol{v}) d x \geqq \Lambda_{1} \int_{\mathbb{R}^{N}}|\nabla \boldsymbol{v}|^{2} d x
$$

for all $v \in \mathscr{D}\left(\mathbb{R}^{N} ; \mathbb{C}^{N}\right)$, and the assertion follows.

(b) $\Lambda_{1} \geqq \Lambda$. This is a standard cut-off argument. Set $\delta>0$ and choose $\boldsymbol{w}=e^{i \omega \cdot x} \boldsymbol{p}(x), \omega \in Y^{*}, \boldsymbol{p} \in \mathscr{H}_{\#}^{1}$ such that

$$
\int_{Y}(\mathbb{L} \nabla w, \nabla w) d x<\left(\Lambda_{1}+\delta\right) \int_{Y}|\nabla w|^{2} d x .
$$

Note that for $z \in \mathbb{Z}^{N}$ one has

$$
\int_{z+Y}(\mathbb{L}(x) \nabla \boldsymbol{w}, \nabla \boldsymbol{w}) d x=\int_{Y}(\mathbb{L}(x) \nabla w, \nabla w) d x .
$$

Let $k \in \mathbb{N}$ and let $\eta \in \mathscr{D}\left(\mathbb{R}^{N}\right)$ be such that $\eta \equiv 1$ on $A_{k}=(-k ; k)^{N}, \eta \equiv 0$ outside $A_{k+1}=(-(k+1), k+1)^{N},|\nabla \eta| \leqq C_{1}$; let $v=\eta w$. For $x \in A_{k+1} \backslash A_{k}$ we have $\nabla v=\eta \nabla w+w \otimes \nabla \eta$ and therefore

$$
\left|\int_{z+Y}(\mathbb{L} \nabla \boldsymbol{v}, \nabla \boldsymbol{v}) d x\right| \leqq C_{2} \int_{Y}\left\{|\boldsymbol{w}|^{2}+|\nabla \boldsymbol{w}|^{2}\right\} d x,
$$

for $z \in\{-(k+1), \ldots, k\}^{N} \backslash\{-k, \ldots, k-1\}^{N}$.

If $\omega=0$, we may assume that $\int_{Y} w d x=0$, so that

$$
\int_{Y}|\boldsymbol{w}|^{2} d x \leqq c \int_{Y}|\nabla \boldsymbol{w}|^{2} d x
$$

by the Poincaré inequality. If $\omega \neq 0$, then (4.13) follows from Lemma 4.1 (with $c=C(\omega)^{-1}$ ). We obtain

$$
\begin{aligned}
\int_{\mathbb{R}^{N}}(\mathbb{L} \nabla \boldsymbol{v}, \nabla \boldsymbol{v}) d x & \leqq(2 k)^{N} \int_{Y}(\mathbb{L} \nabla \boldsymbol{w}, \nabla \boldsymbol{w}) d x+\left[(2(k+1))^{N}-(2 k)^{N}\right] c \int_{Y}|\nabla \boldsymbol{w}|^{2} d x \\
& \leqq\left\{(2 k)^{N}\left(\Lambda_{1}+\delta\right)+\left[(2(k+1))^{N}-(2 k)^{N}\right] c\right\} \int_{\mathrm{Y}}|\nabla \boldsymbol{w}|^{2} d x, \\
\int_{\mathbb{R}^{N}}|\nabla \boldsymbol{v}|^{2} \mathrm{dx} & \geqq \int_{\mathrm{A}_{\mathrm{k}}}|\nabla \boldsymbol{v}|^{2} \mathrm{dx} \cdot(2 \mathrm{k})^{N} \int_{\mathrm{Y}}|\nabla \boldsymbol{w}|^{2} \mathrm{dx}
\end{aligned}
$$

and hence

$$
\Lambda \leqq\left(\Lambda_{1}+\delta\right)+\left(\left(1+k^{-1}\right)^{N}-1\right) c .
$$

Therefore, $\Lambda \leqq \Lambda_{1}+\delta^{*}$ for all $\delta^{*}>0$ and the assertion follows. (c) $\Lambda_{2} \geqq \Lambda_{1}$. This is analogous to part (a). For $\omega \in I=\left\{0, \frac{2 \pi}{k}, \ldots, 2 \pi\left(\frac{k-1}{k}\right)\right\}^{N}$,
and $v=e^{i \omega^{\prime} \cdot x} q, q \in \mathscr{H}_{k \#}^{1}$, define

$$
\tilde{\boldsymbol{v}}_{\omega}(x)=\sum_{z \in\{0, \ldots, k-1\}^{N}} e^{-i\left(\omega+\omega^{\prime}\right) \cdot z} v(x+z) .
$$


A calculation shows that for $z \in \mathbb{Z}^{N}$,

so that

$$
\tilde{\boldsymbol{v}}_{\omega}(x+z)=e^{i\left(\omega+\omega^{\prime}\right) \cdot z} \tilde{\boldsymbol{v}}_{\omega}(x),
$$

$$
\tilde{\boldsymbol{v}}_{\omega}(x)=e^{i\left(\omega+\omega^{\prime}\right) \cdot x} p(x),
$$

with $p \in \mathscr{H}_{\sharp}^{1}$. Analogously to (4.10) one establishes

$$
\int_{k Y}(\mathbb{V} \nabla v, \nabla v) d x=k^{-N} \int_{Y} \sum_{\omega \in I}\left(\mathbb{v} \nabla \tilde{\boldsymbol{v}}_{\omega}, \nabla \tilde{\boldsymbol{v}}_{\omega}\right) d x,
$$

and the proof is finished in the same way as in part (a).

(d) $\Lambda_{1} \geqq \Lambda_{3}$. Together with the obvious inequality $\Lambda_{3} \geqq \Lambda_{2}$ and part (c) this will finish the proof of the lemma. Let $w=e^{i \omega \cdot x} p, p \in \mathscr{H}_{\#}$ and remark that

$$
\begin{gathered}
k^{-N} \int_{k Y}(\mathbb{L} \nabla \boldsymbol{w}, \nabla \boldsymbol{w}) d x=\int_{Y}(\mathbb{L} \nabla \boldsymbol{w}, \nabla \boldsymbol{w}) d x, \\
k^{-N} \int_{k Y}|\nabla \boldsymbol{w}|^{2} d x=\int_{Y}|\nabla \boldsymbol{w}|^{2} d x, \quad k^{-N} \int_{k Y}|\boldsymbol{w}|^{2} d x=\int_{Y}|\boldsymbol{w}|^{2} d x .
\end{gathered}
$$

For any $a \in \mathbb{R}$ let $[a]$ denote the largest integer less than or equal to $a$, set

$$
\omega^{\prime}=\frac{2 \pi}{k}\left(\left[2 k \pi \omega_{1}\right], \ldots,\left[2 \pi k \omega_{N}\right]\right),
$$

and set $\boldsymbol{v}^{(k)}=e^{i \omega^{\prime} \cdot x} \boldsymbol{p}$. Then $\boldsymbol{v}^{(k)} \in \mathscr{H}_{k \#}^{1}$ and

$$
\begin{aligned}
\boldsymbol{v}^{(k)} & =e^{i\left(\omega^{\prime}-\omega\right) \cdot x} \boldsymbol{w}, \\
\nabla \boldsymbol{v}^{(k)} & =e^{i\left(\omega^{\prime}-\omega\right) \cdot x}\left(\nabla \boldsymbol{w}+i\left(\omega^{\prime}-\omega\right)^{*} \otimes \boldsymbol{w}\right) .
\end{aligned}
$$

It follows that as $k \rightarrow \infty$,

$$
\begin{gathered}
k^{-N} \int_{k Y}\left|\left(\mathbb{\|} \nabla \boldsymbol{v}^{(k)}, \nabla \boldsymbol{v}^{(k)}\right)-(\mathbb{\|} \nabla \boldsymbol{w}, \nabla \boldsymbol{w})\right| d x \rightarrow 0, \\
\left.k^{-N} \int_{k Y}|| \nabla \boldsymbol{v}^{(k)}\right|^{2}-|\nabla \boldsymbol{w}|^{2} \mid d x \rightarrow 0 .
\end{gathered}
$$

Therefore

$$
Q(\nabla w ; Y) \geqq \liminf _{k \rightarrow \infty} Q\left(\nabla v^{(k)} ; k Y\right) \geqq \Lambda_{3} .
$$

The assertion follows by taking the infimum over $w$.

\subsection{Proof of Theorem 3.3}

(i) We have only to prove that $\Lambda_{4}=\Lambda_{5}$ and $\Lambda_{6} \leqq \alpha_{11}$.

(a) We first show that $\Lambda_{4} \geqq \Lambda_{5}$. Let $a \in \mathbb{C}^{N}, \boldsymbol{b} \in \mathbb{R}^{N}$ and $q \in \mathscr{H}_{\#}^{1}$ be given with $|\boldsymbol{a} \otimes \boldsymbol{b}+\nabla \boldsymbol{q}| \neq 0$. For $s>0$ let

$$
v_{s}(x)=e^{i s b \cdot x}\left\{\frac{1}{i_{s}} a+q(x)\right\},
$$

By definition of $\Lambda_{5}, \lim _{s \rightarrow 0} Q\left(\nabla v_{s} ; Y\right) \geqq \Lambda_{5}$. On the other hand,

$$
\nabla \boldsymbol{v}_{\boldsymbol{s}}=e^{i s b \cdot x}\{\boldsymbol{a} \otimes \boldsymbol{b}+i s \boldsymbol{q} \otimes \boldsymbol{b}+\nabla \boldsymbol{q}\} \rightarrow \boldsymbol{a} \otimes \boldsymbol{b}+\nabla \boldsymbol{q}
$$


in $L^{2}$ as $s \rightarrow 0$. Therefore,

$$
Q(\boldsymbol{a} \otimes \boldsymbol{b}+\nabla \boldsymbol{q} ; Y)=\lim _{s \rightarrow 0} Q\left(\nabla \boldsymbol{v}_{s} ; Y\right) \geqq \Lambda_{5},
$$

and hence $\Lambda_{4} \geqq \Lambda_{5}$.

(b) To prove the opposite inequality $\Lambda_{4} \leqq \Lambda_{5}$ let $\boldsymbol{v}^{(n)}=e^{i \omega_{n} \cdot x} \boldsymbol{q}^{(n)}$ be a minimizing sequence for $\Lambda_{5}$. We have $\omega_{n} \rightarrow 0$ and may assume that $\left|\nabla v^{(n)}\right|_{L^{2}}=1$. Set $M_{n}=\int_{Y} q^{(n)} d x$. Then

so that

$$
\nabla \boldsymbol{v}^{(n)}=e^{i \omega_{n} \cdot x}\left\{i \boldsymbol{M}_{n} \otimes \omega_{n}+i\left(\boldsymbol{q}^{(n)}-\boldsymbol{M}_{n}\right) \otimes \omega_{n}+\nabla \boldsymbol{q}^{(n)}\right\},
$$

$$
1=\left\|\nabla \boldsymbol{v}^{(n)}\right\|_{L^{2}}^{2}=\left|\boldsymbol{M}_{n} \otimes \omega_{n}\right|^{2}+\left\|i\left(\boldsymbol{q}^{(n)}-\boldsymbol{M}_{n}\right) \otimes \omega_{n}+\nabla \boldsymbol{q}^{(n)}\right\|_{L^{2}}^{2}
$$

by $L^{2}$ orthogonality since

$$
\int_{Y}\left(\boldsymbol{q}^{(n)}-\boldsymbol{M}_{n}\right) d x=\int_{Y} \nabla \boldsymbol{q}^{(n)} d x=0 .
$$

From Lemma 4.1(ii) it follows that (for sufficiently small $\omega_{n}$ ) $\left\|\boldsymbol{q}^{(n)}-\boldsymbol{M}_{n}\right\|_{H^{1}} \leqq C$, and hence

Now

$$
\nabla \boldsymbol{v}^{(n)}-\boldsymbol{e}^{i \omega_{n} \cdot x}\left\{i \boldsymbol{M}_{n} \otimes \omega_{n}+\nabla \boldsymbol{q}^{(n)}\right\} \rightarrow 0 \quad \text { in } L^{2} .
$$

$$
Q\left(e^{i \omega_{n} \cdot x}\left\{i \boldsymbol{M}_{n} \otimes \omega_{n}+\nabla \boldsymbol{q}^{(n)}\right\} ; Y\right)=Q\left(i \boldsymbol{M}_{n} \otimes \omega_{n}+\nabla \boldsymbol{q}^{(n)} ; Y\right) \geqq \Lambda_{4}
$$

by definition of $\Lambda_{4}$, and therefore $\Lambda_{5} \geqq \Lambda_{4}$.

(c) To show that $\Lambda_{6} \leqq \alpha_{\Perp}$, we use highly oscillating test functions which concentrate near $y \in Y$. Recall that $y \in Y$ is a Lebesgue point of $\amalg$ if

$$
\lim _{\mathrm{r} \rightarrow 0} \frac{1}{\operatorname{meas} B_{r}(y)} \int_{\mathrm{B}_{\mathrm{r}}(\mathrm{y})}|\mathbb{U}(x)-\Perp(y)| d x=0,
$$

and that almost every point of $Y$ is a Lebesgue point since $\mathbb{L} \in L^{\infty}$.

Fix a Lebesgue point $y$, fix $\delta>0$, choose $r>0$ such that

$$
\frac{1}{\operatorname{meas} B_{r}(y)} \int_{B_{r}(y)}|\Perp(x)-\Perp(y)| d x<\delta
$$

and choose $\psi \in \mathscr{D}\left(B_{r}(y)\right)$ with $\int_{B_{r}(y)} \psi^{2} d x=1$ and $\psi^{2} \leqq 4 /$ meas $B_{r}(y)$. Finally choose $a^{*}, b^{*} \in \mathbb{R}^{N},\left|a^{*}\right|=\left|b^{*}\right|=1$ such that

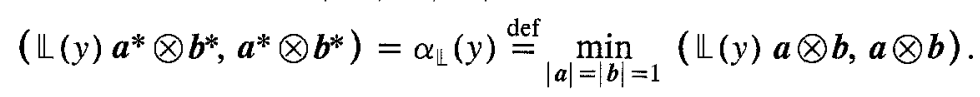

Consider $v^{(j)}(x)=\frac{1}{j} \psi(x) e^{i j\left(x \cdot b^{*}\right)} a^{*}$. Clearly $v^{(j)} \rightarrow 0, \nabla \boldsymbol{v}^{(j)}-i \boldsymbol{a}^{*} \psi(x) \otimes \boldsymbol{b}^{*}$ $e^{i j\left(x \cdot b^{*}\right)} \rightarrow 0$ in $L^{2}$ and hence, by also using (4.14),

$$
\begin{aligned}
\int_{\boldsymbol{Y}}\left(\mathbb{\|} \nabla \boldsymbol{v}^{(\mathrm{j})}, \nabla \boldsymbol{v}^{(j)}\right) d x \rightarrow & \int_{\boldsymbol{Y}} \psi^{2}(x)\left(\mathbb{L}(x) \boldsymbol{a}^{*} \otimes \boldsymbol{b}^{*}, \boldsymbol{a}^{*} \otimes \boldsymbol{b}^{*}\right) d x \\
& \leqq\left(\mathbb{L}(y) \boldsymbol{a}^{*} \otimes \boldsymbol{b}^{*}, \boldsymbol{a}^{*} \otimes \boldsymbol{b}^{*}\right)+4 \delta \leqq \alpha_{\mathbb{L}}(y)+4 \delta .
\end{aligned}
$$

It follows that $\Lambda_{6} \leqq \alpha_{\mathbb{L}}(y)$ whenever $y$ is a Lebesgue point of $\mathbb{L}$ and the assertion is proved. 
(ii) The functionals $\mathscr{L}_{k}(q ; F)$ are strongly continuous and quadratic. Then the assertions follow immediately since for all $q \in \mathscr{H}_{k \#}^{1}$, one has, thanks to the assumption that $A \geqq 0$ and the Lemma 4.2,

$$
\mathscr{L}_{k}(\boldsymbol{q}, 0)=\int_{k Y}(\Perp(x) \nabla \boldsymbol{q}, \nabla \boldsymbol{q}) d x \geqq 0 .
$$

(iii) Obviously the set $N_{k}$ is a (closed) subspace. For later use, let us also remark that for $\boldsymbol{q} \in N_{k}, \boldsymbol{a} \in \mathbb{C}^{N}, \boldsymbol{b} \in \mathbb{R}^{N}$ and $\boldsymbol{q}^{\perp} \in N_{k}^{\perp}$ we have

$$
\begin{aligned}
& \int_{k Y} \operatorname{Re}(\Perp(x) a \otimes b, \nabla \boldsymbol{q}) d x=0, \\
& \int_{k Y} \operatorname{Re}\left(\Perp(x) \nabla \boldsymbol{q}, \nabla \boldsymbol{q}^{\perp}\right) d x=0 .
\end{aligned}
$$

Indeed, since for all $\lambda \in \mathbb{R}$ and $q \in N_{k}$,

$$
\begin{aligned}
0 & \leqq \int_{k Y}(\mathbb{L}(x)(\boldsymbol{a} \otimes \boldsymbol{b}+\lambda \nabla \boldsymbol{q}), \boldsymbol{a} \otimes \boldsymbol{b}+\lambda \nabla \boldsymbol{q}) d x \\
& \leqq \int_{k Y}(\mathbb{L}(x) \boldsymbol{a} \otimes \boldsymbol{b}, \boldsymbol{a} \otimes \boldsymbol{b}) d x+2 \lambda \int_{k Y} \operatorname{Re}(\mathbb{L}(x) \boldsymbol{a} \otimes \boldsymbol{b}, \nabla \boldsymbol{q}),
\end{aligned}
$$

one must have (4.15). In the same way one deduces (4.16). By (3.6) Gårding's inequality holds (on the torus $\mathbb{J}^{N}=\mathbb{R}^{N} /(k \mathbb{Z})^{N}$ ), i.e., there exist constants $\mu_{k}$, $\lambda>0$, such that

$$
\int_{k Y}(\mathbb{L}(x) \nabla \boldsymbol{q}, \nabla \boldsymbol{q}) d x \geqq \frac{1}{2} \lambda \int_{k Y}|\nabla \boldsymbol{q}|^{2} d x-\mu_{k} \int_{k Y}|\boldsymbol{q}|^{2} d x .
$$

The standard reasoning by contradiction then shows that $N_{k}$ is finite-dimensional and that there exist $c_{k}>0$ such that

$$
\int_{k Y}(\mathbb{L}(x) \nabla \boldsymbol{q}, \nabla \boldsymbol{q}) d x \geqq c_{k} \int_{k Y}|\nabla \boldsymbol{q}|^{2} d x \quad \text { for all } \boldsymbol{q} \in N_{k}^{\perp} .
$$

(iv) Fix $k$ and $F$. Let $q^{(j)} \in \mathscr{H}_{k \notin}^{1}$ be a minimizing sequence for $\left(M_{k} F, F\right)$, i.e.,

$$
\mathscr{L}_{k}\left(\boldsymbol{q}^{(j)} ; F\right) \rightarrow\left(M_{k} F, F\right)
$$

Observe that for $z \in \mathbb{Z}, \mathscr{L}_{k}\left(q^{(j)}(\cdot+z) ; F\right)=\mathscr{L}_{k}\left(q^{(j)} ; F\right)$. Let

$$
\tilde{\boldsymbol{q}}^{(j)}(x)=\frac{1}{k^{N}} \sum_{z \in\{0, \ldots, k-1\}^{N}} \boldsymbol{q}^{(j)}(x+z) ;
$$

then $\tilde{\boldsymbol{q}}^{(j)} \in \mathscr{H}_{\#}^{1}$, and by convexity of $\mathscr{L}_{k}$,

$$
\mathscr{L}\left(\tilde{\boldsymbol{q}}^{(j)} ; F\right)=\mathscr{L}_{k}\left(\tilde{\boldsymbol{q}}^{(j)} ; F\right) \leqq \frac{1}{k^{N}} \sum_{z \in\{0, \ldots, k-1\}^{N}} \mathscr{L}_{k}\left(\boldsymbol{q}^{(j)} ; F\right)
$$

Hence

$$
(M F, F) \leqq\left(\mathbb{M}_{k} F, F\right) .
$$

As the opposite inequality is obvious, the result follows. 
(v) Note that for $\boldsymbol{q} \in \mathscr{H}_{\#}^{1}, \int_{Y} \nabla \boldsymbol{q} d x=0$. The assertion then follows from (3.18) and the fact that by $L^{2}$-orthogonality,

$$
\int_{Y}|\boldsymbol{a} \otimes \boldsymbol{b}+\nabla \boldsymbol{q}|^{2} d x=|\boldsymbol{a} \otimes \boldsymbol{b}|^{2}+\int_{Y}|\nabla \boldsymbol{q}|^{2} d x \geqq|\boldsymbol{a} \otimes \boldsymbol{b}|^{2},
$$

which implies $\mathscr{L}_{1}(\boldsymbol{a} \otimes \boldsymbol{b} ; \boldsymbol{q}) \geqq \Lambda_{4}|\boldsymbol{a} \otimes \boldsymbol{b}|^{2}$.

(vi) If $\Lambda_{6}>0$, the result follows from (ii) and the coercivity of $\int_{Y}(\mathbb{L}(x) \nabla \boldsymbol{q}, \nabla \boldsymbol{q}) d x$ on $\mathscr{H}_{\#} \frac{1}{\#}$.

(vii) This follows from (ii) by virtue of the compact imbedding of $\mathscr{H}_{\#}^{1}$ into $\mathscr{L}_{\#}^{2}$.

\subsection{Proof of Theorem 3.1}

(i) Let $\varphi \in \mathscr{D}\left(\Omega ; \mathbb{R}^{N}\right)$ be given. Extending $\varphi$ by zero to $\mathbb{R}^{N}$ we obtain from (3.11) after a change of variables that

$$
a^{\varepsilon}(\varphi, \varphi) \geqq \Lambda \int_{\Omega}|\nabla \varphi|^{2} d x,
$$

and so by the Poincare inequality, $a^{\varepsilon}$ is uniformly coercive (in $\varepsilon$ ) on $H_{0}^{1}\left(\Omega ; \mathbb{R}^{N}\right)$. It now follows from the Lax-Milgram theorem that for every $\varepsilon \rightarrow 0$, the problem $\left(\mathrm{P}^{\varepsilon}\right)$ has a unique solution $\boldsymbol{v}^{\varepsilon}$ satisfying (3.13). Passing to a subsequence we have $\boldsymbol{v}^{\varepsilon} \rightarrow \boldsymbol{v}$ weakly in $H^{1}\left(\Omega ; \mathbb{R}^{N}\right)$ with $v-v_{0} \in H_{0}^{1}\left(\Omega ; \mathbb{R}^{N}\right)$ and $\mathbb{L}(x / \varepsilon) \nabla \boldsymbol{v}^{\varepsilon} \rightarrow \sigma$ weakly in $L^{2}$ as $\varepsilon \rightarrow 0$.

(ii) Note that Theorem 3.3(i) and again the Lax-Milgram theorem imply that (3.17) has a solution $\tau \in H_{\#}^{1}$ unique up to a constant. Hence $M$ is well defined by (3.16). Moreover $M$ is symmetric since $\amalg$ is. To see that (3.18) gives an equivalent characterization of $M$ note that by Theorem 3.3 (ii) the infimum in (3.18) is attained. The minimizer has to satisfy (3.17) and is hence unique (up to a constant). Using (3.17) once more one sees that the right-hand side of (3.18) equals $(M F, F)$ with $M$ defined by (3.18), (3.17). Finally note that $M$ is determined by the values of $(M F, F)$ for all $F$ since $M$ is symmetric. Inequality (3.19) follows from Theorem 3.3(v). The proof that $\sigma=\mathbb{M} \nabla v$ and that therefore (3.14) holds is identical to TARTAR's proof (see [BLP 78] for the scalar case and [SP 80] for classical elasticity).

(iii) This is a simple exercice.

\subsection{Proof of Theorem 3.4 (i) when $\Lambda>0$}

In this case $\Gamma$-convergence can be deduced in a standard way from the convergence results for the Euler-Lagrange equation obtained in Theorem 3.1. We provide the details for the convenience of the reader.

According to Definition 2.1 we have to show

(i) If $\boldsymbol{u}^{\varepsilon} \in \mathbb{K}_{\boldsymbol{v}_{0}}$ and $\boldsymbol{u}^{\varepsilon}-\boldsymbol{u}$ in $H^{1}\left(\Omega ; \mathbb{R}^{N}\right)$, then

$$
\liminf _{\varepsilon \rightarrow 0} a^{\varepsilon}\left(\boldsymbol{u}^{\varepsilon}, \boldsymbol{u}^{\varepsilon}\right) \geqq a(\boldsymbol{u}, \boldsymbol{u}) .
$$


(ii) For every $u \in \mathbb{K}_{\boldsymbol{v}_{0}}$ there is a sequence $\boldsymbol{u}^{\varepsilon} \in \mathbb{K}_{v_{0}}, \boldsymbol{u}^{\varepsilon} \rightarrow \boldsymbol{u}$ in $H^{1}\left(\Omega ; \mathbb{R}^{N}\right)$, with

$$
\lim _{\varepsilon \rightarrow 0} a^{\varepsilon}\left(\boldsymbol{u}^{\varepsilon}, \boldsymbol{u}^{\varepsilon}\right)=a(u, u) .
$$

To show (ii) fix $u \in \mathbb{K}_{v_{0}}$ and let

$$
f=\operatorname{div}(\mathbb{M} \nabla \boldsymbol{u}) \in H^{-1}\left(\Omega ; \mathbb{R}^{N}\right),
$$

where $\mathbb{M}$ is the homogenized tensor in (3.18).

Let $\boldsymbol{v}^{\varepsilon} \in \mathbb{K}_{v_{0}}$ be the (unique) weak solution of

$$
a^{\varepsilon}\left(\boldsymbol{v}^{\varepsilon}, \varphi\right)+\langle f, \varphi\rangle=0 \quad \text { for all } \varphi \in H_{0}^{1}\left(\Omega ; \mathbb{R}^{N}\right) .
$$

It follows from Theorem 3.1 and its proof that

$$
\begin{gathered}
\boldsymbol{v}^{\varepsilon} \rightarrow \boldsymbol{v} \quad \text { (weakly) in } H^{1}\left(\Omega ; \mathbb{R}^{N}\right), \\
\sigma^{\varepsilon} \stackrel{\text { def }}{=} \amalg\left(\frac{x}{\varepsilon}\right) \nabla \boldsymbol{v}^{\varepsilon} \rightarrow \sigma \stackrel{\text { def }}{=} \mathbb{M} \nabla \boldsymbol{v} \quad \text { (weakly) in } L^{2} .
\end{gathered}
$$

Moreover $v$ is the unique solution in $\mathbb{K}_{v_{0}}$ of

$$
a(v, \varphi)+\langle f, \varphi\rangle=0 \quad \text { for all } \varphi \in H_{0}^{1}\left(\Omega ; \mathbb{R}^{N}\right)
$$

and hence, by the definition of $f, v=u$. Now by (4.20) and (4.21),

$$
\begin{aligned}
a^{\varepsilon}\left(\boldsymbol{v}^{\varepsilon}, \boldsymbol{v}^{\varepsilon}\right) & =a^{\varepsilon}\left(\boldsymbol{v}^{\varepsilon}, \boldsymbol{v}^{\varepsilon}-\boldsymbol{v}_{0}\right)+a^{\varepsilon}\left(\boldsymbol{v}^{\varepsilon}, \boldsymbol{v}_{0}\right) \\
& =-\left\langle f, \boldsymbol{v}^{\varepsilon}-\boldsymbol{v}_{0}\right\rangle+\left(\sigma^{\varepsilon}, \nabla \boldsymbol{v}_{0}\right) \\
& \rightarrow-\left\langle f, \boldsymbol{u}-\boldsymbol{v}_{0}\right\rangle+\left(\sigma, \nabla \boldsymbol{v}_{0}\right) \\
& =a\left(\boldsymbol{u}, \boldsymbol{u}-\boldsymbol{v}_{0}\right)+a\left(\boldsymbol{u}, \boldsymbol{v}_{0}\right)=a(\boldsymbol{u}, \boldsymbol{u}) .
\end{aligned}
$$

This finishes the proof of (ii).

To show (i), let $\boldsymbol{u}$ and $\boldsymbol{f}$ be as above, let $\boldsymbol{u}^{\varepsilon} \in \mathbb{K}_{\boldsymbol{v}_{0}}$ and let $\boldsymbol{u}^{\varepsilon} \rightarrow \boldsymbol{u}$ in $H^{1}\left(\Omega ; \mathbb{R}^{N}\right)$. Now (4.19) implies that $v^{\varepsilon}$ is the (unique) minimizer in $\mathbb{K}_{v_{0}}$ of

$$
I^{\varepsilon}(u)=\frac{1}{2} a^{\varepsilon}(u, u)-\left\langle f, u-v_{0}\right\rangle .
$$

Therefore (4.20) and (4.23) yield that

$$
\liminf _{\varepsilon \rightarrow 0} I^{\varepsilon}\left(\boldsymbol{u}^{\varepsilon}\right) \geqq \liminf _{\varepsilon \rightarrow 0} I^{\varepsilon}\left(\boldsymbol{v}^{\varepsilon}\right)=I(\boldsymbol{u}) .
$$

Now (i) follows since $\left\langle f, \boldsymbol{u}^{\varepsilon}-\boldsymbol{v}_{0}\right\rangle \rightarrow\left\langle f, \boldsymbol{v}_{0}\right\rangle$.

\subsection{Proof of Theorem 3.4(i) when $\Lambda=0$ and $\Lambda_{6}>0$}

Following the Definition 2.1, we verify condition (i) and condition (ii).

Condition (i). Let $\boldsymbol{u}^{\varepsilon} \in \mathbb{K}_{\boldsymbol{v}_{0}}$ and let $\boldsymbol{u}^{\varepsilon} \rightarrow \boldsymbol{u} \in \mathbb{K}_{\boldsymbol{v}_{0}}$ weakly in $H^{1}\left(\Omega ; \mathbb{R}^{N}\right)$. Then, in particular,

$$
\limsup _{\varepsilon \rightarrow 0}\left\|\boldsymbol{u}^{\varepsilon}\right\|_{H^{1}} \leqq C \text {. }
$$


For $\delta>0$, set $\mathbb{L}^{\delta}=\mathbb{L}+\delta$ Id; then $\Lambda\left(\mathbb{L}^{\delta}\right)=\delta>0$. Applying Theorem 3.4(i) to

$$
a^{\delta, \varepsilon}(\boldsymbol{u}, \boldsymbol{u})=a^{\varepsilon}(u, u)+\delta\|\nabla u\|_{L^{2}}^{2}=\int_{\Omega}\left(\mathbb{L}^{\delta}\left(\frac{x}{\varepsilon}\right) \nabla u, \nabla u\right) d x
$$

we have

$$
\liminf _{\varepsilon \rightarrow 0} a^{\delta, \varepsilon}\left(\boldsymbol{u}^{\varepsilon}, \boldsymbol{u}^{\varepsilon}\right) \geqq a^{\delta}(\boldsymbol{u}, \boldsymbol{u}) \geqq a(\boldsymbol{u}, \boldsymbol{u}) .
$$

On the other hand,

$$
\limsup _{\varepsilon \rightarrow 0}\left|a^{\delta, \varepsilon}\left(\boldsymbol{u}^{\varepsilon}, \boldsymbol{u}^{\varepsilon}\right)-a^{\varepsilon}\left(\boldsymbol{u}^{\varepsilon}, \boldsymbol{u}^{\varepsilon}\right)\right| \leqq \delta \limsup _{\varepsilon \rightarrow 0}\left\|\boldsymbol{u}^{\varepsilon}\right\|_{H^{1}}^{2} \leqq \delta C^{2} .
$$

Thus

$$
\liminf _{\varepsilon \rightarrow 0} a^{\varepsilon}\left(\boldsymbol{u}^{\varepsilon}, \boldsymbol{u}^{\varepsilon}\right) \geqq a(\boldsymbol{u}, \boldsymbol{u})-\delta C^{2} .
$$

Since $\delta>0$ is arbitrary, the result follows.

Condition (ii). This is more complicated since we have to explicitly construct the desired sequence. By using linearity we achieve this in two steps, considering a smooth limit $\boldsymbol{u}$ first. While the details of that procedure are well known to experts in homogenization we include them here to keep the exposition selfcontained. Since $\Lambda_{6}>0$, Theorem 3.3 (vi) implies that for every $N \times N$ matrix $F$ there exists a unique $r_{F} \in \mathscr{H}_{\#}^{1}$ with $\int_{Y} r_{F}(x) d x=0$ such that for all $\varphi \in \mathscr{H}_{\#}^{1}$,

Moreover,

$$
\int_{Y}\left(\Perp(x)\left(F+\nabla r_{F}\right), \nabla \varphi\right) d x=0 .
$$

$$
\left\|\nabla \boldsymbol{r}_{F}\right\|_{L_{\text {妾 }}^{2}} \leqq C|F| .
$$

Define a 3-tensor $\rho(x)$ and a 4-tensor $\mathbb{R}(x)$ by

$$
\rho(x) F=r_{F}(x), \quad \mathbb{R}(x) F=\nabla r_{F}(x) .
$$

It follows from (4.24) that

$$
\int_{Y}|\mathbb{R}(x)|^{2} d x \leqq C^{2}, \quad \int_{Y} \rho(x) d x=0, \quad \int_{Y}|\rho(x)|^{2} d x \leqq C^{2} .
$$

Step 1. Let $u \in C^{2}\left(\bar{\Omega} ; \mathbb{R}^{N}\right)$ and set

Then

$$
\boldsymbol{u}^{\varepsilon}(x)=\boldsymbol{u}(x)+\varepsilon \rho\left(\frac{x}{\varepsilon}\right) \nabla \boldsymbol{u}(x) .
$$

$$
\nabla \boldsymbol{u}^{\varepsilon}=\nabla \boldsymbol{u}+\mathbb{R}\left(\frac{x}{\varepsilon}\right) \nabla \boldsymbol{u}+\varepsilon \rho\left(\frac{x}{\varepsilon}\right) \nabla^{2} \boldsymbol{u} .
$$

By (4.25), $\boldsymbol{u}^{\varepsilon} \rightarrow \boldsymbol{u}$ in $L^{2}\left(\Omega ; \mathbb{R}^{N}\right)$ strongly and

$$
\limsup _{\varepsilon \rightarrow 0}\left\|\nabla \boldsymbol{u}^{\varepsilon}\right\|_{L^{2}} \leqq C\|\nabla \boldsymbol{u}\|_{L^{2}} .
$$

As $\varepsilon \rightarrow 0$ the last term on the right of (4.26) converges to zero strongly in $L^{2}$, and hence

$$
\lim _{\varepsilon \rightarrow 0} a^{\varepsilon}\left(\boldsymbol{u}^{\varepsilon}, \boldsymbol{u}^{\varepsilon}\right)=\lim _{\varepsilon \rightarrow 0} \int_{\Omega} f\left(x, \frac{x}{\varepsilon}\right) d x,
$$


where

$$
f(x, y)=(\mathbb{L}(y)[\nabla \boldsymbol{u}(x)+\mathbb{R}(y) \nabla \boldsymbol{u}(x)], \nabla \boldsymbol{u}(x)+\mathbb{R}(y) \nabla \boldsymbol{u}(x)) .
$$

Note that $\boldsymbol{u}^{\varepsilon}-\boldsymbol{u}$ in $H^{1}\left(\Omega ; \mathbb{R}^{N}\right)$, and by (3.18) and the definition of $\mathbb{R}$,

Moreover,

$$
\bar{f}(x)=\int_{Y} f(x, y) d y=(\mathbb{M} \nabla \boldsymbol{u}(x), \nabla \boldsymbol{u}(x)) .
$$

$$
\left|f\left(x_{1}, y\right)-f\left(x_{2}, y\right)\right| \leqq C(\boldsymbol{u})\left|x_{1}-x_{2}\right|(1+|\mathbb{R}(y)|)^{2} ;
$$

from this it is easily shown that

$$
\lim _{\varepsilon \rightarrow 0} \int_{\Omega} f\left(x, \frac{x}{\varepsilon}\right) d x=\int_{\Omega} \bar{f}(x) d x=a(\boldsymbol{u}, \boldsymbol{u}) .
$$

The desired conclusion follows.

For future reference we remark that a similar argument implies that

$$
\begin{aligned}
\limsup _{\varepsilon \rightarrow 0} & \int_{\Omega} \psi^{2}(x)\left|\nabla \boldsymbol{u}^{\varepsilon}-\nabla \boldsymbol{u}\right|^{2} d x \leqq \limsup _{\varepsilon \rightarrow 0} \int_{\Omega} \psi^{2}(x)\left|\mathbb{R}\left(\frac{x}{\varepsilon}\right)\right|^{2}|\nabla \boldsymbol{u}|^{2} d x \\
\leqq & \left(\int_{Y}|\mathbb{R}(y)|^{2} d y\right) \int_{\Omega} \psi^{2}(x)|\nabla \boldsymbol{u}|^{2} d x \leqq C^{2} \int_{\Omega} \psi^{2}(x)|\nabla \boldsymbol{u}|^{2} d x .
\end{aligned}
$$

Step 2. Let $\boldsymbol{u} \in \mathbb{K}_{v_{0}}$ be arbitrary. Choose $\boldsymbol{u}^{\delta} \in C^{2}\left(\bar{\Omega} ; \mathbb{R}^{N}\right)$ such that $\boldsymbol{u}^{\delta} \rightarrow \boldsymbol{u}$ strongly in $H^{1}\left(\Omega ; \mathbb{R}^{N}\right)$ (this is possible since $\Omega$ is Lipschitzian and bounded and one thus has an extension operator $E: H^{1}(\Omega) \rightarrow H^{1}\left(\mathbb{R}^{N}\right)$ see [ST 70]). Choose $\psi^{\delta} \in C^{\infty}(\bar{\Omega})$ such that

$$
\begin{aligned}
\psi^{\delta}(x) & = \begin{cases}1 & \text { if } \operatorname{dist}(x, \partial \Omega)<\delta, \\
0 & \text { if } \operatorname{dist}(x, \partial \Omega)>2 \delta,\end{cases} \\
\sup _{x \in \bar{\Omega}}\left|\nabla \psi^{\delta}(x)\right| & \leqq A \delta^{-1} \quad \text { with } A \text { independent of } \delta .
\end{aligned}
$$

By the previous step, there are $\boldsymbol{u}^{\varepsilon, \delta} \in C^{2}\left(\bar{\Omega} ; \mathbb{R}^{N}\right)$ such that

$$
\lim _{\varepsilon \rightarrow 0} a^{\varepsilon}\left(\boldsymbol{u}^{\varepsilon, \delta}, \boldsymbol{u}^{\varepsilon, \delta}\right)=a\left(\boldsymbol{u}^{\delta}, \boldsymbol{u}^{\delta}\right),
$$

and (4.26), (4.27) imply that

$$
\begin{gathered}
\limsup _{\varepsilon \rightarrow 0}\left\|\boldsymbol{u}^{\varepsilon, \delta}\right\|_{H^{1}} \leqq C\left\|\boldsymbol{u}^{\delta}\right\|_{H^{1}}, \\
\boldsymbol{u}^{\varepsilon, \delta} \rightarrow \boldsymbol{u}^{\delta} \quad \text { in } L^{2}\left(\Omega ; \mathbb{R}^{N}\right) \text { strongly, } \\
\boldsymbol{u}^{\varepsilon, \delta} \rightarrow \boldsymbol{u}^{\delta} \quad \text { in } H^{1}\left(\Omega ; \mathbb{R}^{N}\right) \text { weakly. }
\end{gathered}
$$

Define $z^{\varepsilon, \delta}=\left(1-\psi^{\delta}\right) \boldsymbol{u}^{\varepsilon, \delta}+\psi^{\delta} \boldsymbol{u}$ and observe that $z^{\varepsilon, \delta} \in \mathbb{K}_{\boldsymbol{v}_{0}}$. Now

$$
\nabla \boldsymbol{z}^{\varepsilon, \delta}=\nabla \boldsymbol{u}^{\varepsilon, \delta}+\psi^{\delta}\left(\nabla \boldsymbol{u}^{\delta}-\nabla \boldsymbol{u}^{\varepsilon, \delta}\right)+\psi^{\delta}\left(\nabla \boldsymbol{u}-\nabla \boldsymbol{u}^{\delta}\right)+\left(\boldsymbol{u}^{\varepsilon, \delta}-\boldsymbol{u}\right) \otimes \nabla \psi^{\delta}
$$

and $\left(\boldsymbol{u}^{\delta}-\boldsymbol{u}^{\varepsilon, \delta}\right) \otimes \nabla \psi^{\delta} \rightarrow 0$ in $L^{2}$ as $\varepsilon \rightarrow 0$. Thus by (4.27), (4.28), (4.29), (4.30) applied to $\psi^{\delta}, \boldsymbol{u}^{\varepsilon, \delta}, \boldsymbol{u}^{\delta}$ one has

$$
\begin{gathered}
\limsup _{\varepsilon \rightarrow 0} a^{\varepsilon}\left(\boldsymbol{z}^{\varepsilon, \delta}, z^{\varepsilon, \delta}\right) \leqq a\left(\boldsymbol{u}^{\delta}, \boldsymbol{u}^{\delta}\right)+C\left(a\left(\boldsymbol{u}^{\delta}, \boldsymbol{u}^{\delta}\right)\right)^{1 / 2} T_{\delta}^{1 / 2}+C T_{\delta}, \\
T_{\delta}=\left\|\psi^{\delta} \nabla \boldsymbol{u}^{\delta}\right\|_{L^{2}}^{2}+\left\|\psi^{\delta}\left(\nabla \boldsymbol{u}-\nabla \boldsymbol{u}^{\delta}\right)\right\|_{L^{2}}^{2}+\left\|\left(\boldsymbol{u}^{\delta}-\boldsymbol{u}\right) \otimes \nabla \psi^{\delta}\right\|_{L^{2}}^{2} .
\end{gathered}
$$


We may assume that $\left\|\boldsymbol{u}-\boldsymbol{u}^{\delta}\right\|_{H^{1}} \leqq C \delta^{2}$ and thus obtain

$$
\limsup _{\delta \rightarrow 0} \limsup _{\varepsilon \rightarrow 0} a^{\varepsilon}\left(z^{\varepsilon, \delta}, z^{\varepsilon, \delta}\right) \leqq a(\boldsymbol{u}, \boldsymbol{u}) .
$$

Consider now the quantity

$$
E(\varepsilon, \delta)=\left(a^{\varepsilon}\left(z^{\varepsilon, \delta}, z^{\varepsilon, \delta}\right)-a(u, u)\right)^{+}+\left\|z^{\varepsilon, \delta}-u\right\| L_{L^{2}}+\left(\left\|z^{\varepsilon, \delta}\right\|_{H^{1}}-C\|u\|_{H^{1}}\right)^{+}
$$

where $a^{+}=a$ if $a>0$, and $a^{+}=0$ otherwise. By the triangle inequality,

$$
\limsup _{\delta \rightarrow 0} \limsup _{\varepsilon \rightarrow 0} E(\varepsilon, \delta) \leqq 0 .
$$

We use now a diagonalization lemma of Aтtouch [A 84, Cor. 1.16] to obtain a function $\delta(\varepsilon)$ such that

$$
\limsup _{\varepsilon \rightarrow 0} E(\varepsilon, \delta(\varepsilon)) \leqq 0 .
$$

Set $\boldsymbol{v}^{\varepsilon}=z^{\varepsilon, \delta(\varepsilon)}$. Then $\boldsymbol{v}^{\varepsilon} \in \mathbb{K}_{v_{0}}$

$$
\begin{gathered}
\limsup _{\varepsilon \rightarrow 0} a^{\varepsilon}\left(v^{\varepsilon}, v^{\varepsilon}\right) \leqq a(u, u), \\
\boldsymbol{v}^{\varepsilon} \rightarrow \boldsymbol{u} \quad \text { strongly in } L^{2}\left(\Omega ; \mathbb{R}^{N}\right), \\
\limsup _{\varepsilon \rightarrow 0}\left\|v^{\varepsilon}\right\|_{H^{1}} \leqq C\|\boldsymbol{u}\|_{H^{1}}<+\infty,
\end{gathered}
$$

and thus $\boldsymbol{v}^{\varepsilon} \rightarrow \boldsymbol{u}$ weakly in $H^{1}\left(\Omega ; \mathbb{R}^{N}\right)$. Now using condition (i) we deduce that also $\liminf _{\varepsilon \rightarrow 0} a^{\varepsilon}\left(v^{\varepsilon}, v^{\varepsilon}\right) \geqq a(u, u)$ and thus obtain the desired result.

\subsection{Proof of Theorem 3.4(ii)}

By Lemma 4.2, $\Lambda_{1}=\Lambda=0$ and thus there exists a sequence $v^{(n)}=e^{i \omega_{n} \cdot x} q^{(n)}$ such that $\boldsymbol{q}^{(n)} \in \mathscr{H}_{\#}^{1}$ and

$$
\left\|\nabla \boldsymbol{v}^{(n)}\right\|_{L^{2}}=1, \quad \lim _{n \rightarrow \infty} Q\left(\nabla \boldsymbol{v}^{(n)} ; Y\right)=0, \quad \omega_{n} \rightarrow \omega \in\left[0,2 \pi\left[^{N} .\right.\right.
$$

If $\omega=0$, then by definition of $\Lambda_{5}$ and Theorem $3.3(\mathrm{i}), \Lambda_{4}=\Lambda_{5}=0$, contradicting the assumption. Thus $\omega \neq 0$. Now by $L^{2}$-orthogonality,

$$
\begin{aligned}
1 & =\left\|\nabla \boldsymbol{v}^{(n)}\right\|_{L^{2}}^{2}=\left\|\left[\int_{Y} \boldsymbol{q}^{(n)} d x\right] \otimes i \omega_{n}+\left(\boldsymbol{q}^{(n)}-\left[\int_{Y} \boldsymbol{q}^{(n)} d x\right]\right) \otimes i \omega_{n}+\nabla \boldsymbol{q}^{(n)}\right\|_{L^{2}}^{2} \\
& =\left\|\left.\left[\int_{Y} \boldsymbol{q}^{(n)} d x\right] \otimes \omega_{n}\right|^{2}+\right\|\left(\boldsymbol{q}^{(n)}-\left[\int_{Y} \boldsymbol{q}^{(n)} d x\right]\right) \otimes i \omega_{n}+\nabla \boldsymbol{q}^{(n)} \|_{L^{2}}^{2}
\end{aligned}
$$

Since $\omega \neq 0, \int_{Y} q^{(n)} d x$ is bounded. Moreover, by Lemma 4.1, $q^{(n)}-\int_{Y} q^{(n)} d x$ and thus $\boldsymbol{q}^{(n)}$ is bounded in $\mathscr{H}_{\#}^{1}$. In particular, (for a subsequence) $\boldsymbol{q}^{(n)} \rightarrow \boldsymbol{q}$ in $L^{2}$ and $\nabla \boldsymbol{v}^{(n)}-\nabla\left(e^{i \omega \cdot x} \boldsymbol{q}^{(n)}\right) \rightarrow 0$ in $L^{2}$. Thus $\lim _{n \rightarrow \infty} Q\left(\nabla\left(e^{i \omega \cdot x} \boldsymbol{q}^{(n)}\right) ; Y\right)=0$ and by lower semicontinuity (Theorem 3.3(ii)) and the fact that $\Lambda_{1}=0$ one has

$$
\mathscr{L}_{1}(q ; 0)=Q\left(\nabla\left(e^{i \omega \cdot x} q\right) ; Y\right)=0 .
$$



hence

We show next that $\boldsymbol{q} \neq 0$. Assume otherwise. Then $\boldsymbol{q}^{(n)} \otimes \nabla e^{i \omega \cdot x} \rightarrow 0$, and

$$
\begin{aligned}
0 & =\lim _{n \rightarrow \infty} Q\left(\nabla\left(e^{i \omega \cdot x} \boldsymbol{q}^{(n)}\right) ; Y\right)=\lim _{n \rightarrow \infty} Q\left(e^{i \omega \cdot x} \nabla \boldsymbol{q}^{(n)} ; Y\right)=\lim _{n \rightarrow \infty} Q\left(\nabla \boldsymbol{q}^{(n)} ; Y\right) \\
& \geqq \Lambda_{6}\left\|\nabla \boldsymbol{q}^{(n)}\right\|^{2} .
\end{aligned}
$$

Thus $\boldsymbol{q}^{(n)} \rightarrow 0$ strongly in $\mathscr{H}_{\#}^{1}$, contradicting the assumption that $\left\|\nabla \boldsymbol{v}^{(n)}\right\|_{L^{2}}=1$.

Since $\Lambda_{1}=0, q$ is in fact a minimizer of $\mathscr{L}_{1}$ and thus satisfies the EulerLagrange equation

$$
\int_{Y}\left(\mathbb{L}(x) \nabla\left(e^{i \omega \cdot x} q\right), \nabla\left(e^{i \omega \cdot x} \hat{\eta}\right)\right) d x=0 \quad \text { for all } \hat{\eta} \in \mathscr{H}_{\#}^{1} .
$$

This in fact implies that

$$
\int_{\mathbb{R}^{N}}\left(\amalg(x) \nabla\left(e^{i \omega \cdot x} q\right), \nabla\left(e^{i \omega \cdot x} \eta\right)\right) d x=0 \quad \text { for all } \eta \in \mathscr{D}\left(\mathbb{R}^{N} ; \mathbb{C}^{N}\right) .
$$

The desired inequality (3.26) follows. To verify (4.31) let $\hat{\eta}(x)=\sum_{z \in \mathbb{Z}^{N}} \eta(x+z)$. Then $\hat{\eta}$ is $Y$-periodic and we have

$$
\left.\int_{\mathbb{R}^{N}}\left(\Perp(x) \nabla e^{i \omega \cdot x} q\right), \nabla\left(e^{i \omega \cdot x} \eta\right)\right) d x=\int_{Y}\left(\mathbb{L}(x) \nabla\left(e^{i \omega \cdot x} q\right), \nabla\left(e^{i \omega \cdot x} \hat{\eta}\right)\right) d x=0 .
$$

Observe finally that the estimate (3.25) is only a restatement of Theorem $3.3(\mathrm{v})$ since $\Lambda_{6} \geqq \Lambda_{4}>0$.

\subsection{Proof of Theorem 3.4 (iii)}

Since $\Lambda_{4}=0$, there exist sequences $F^{(n)}=\boldsymbol{a}^{(n)} \otimes \boldsymbol{b}^{(n)}$ and $\boldsymbol{q}^{(n)} \in \mathscr{H}_{\#}^{1}$ such that

$$
\begin{gathered}
\int_{Y} \boldsymbol{q}^{(n)} d x=0, \\
\int_{Y}\left|F^{(n)}+\nabla \boldsymbol{q}^{(n)}\right|^{2} d x=\left|F^{(n)}\right|^{2}+\int_{Y}\left|\nabla \boldsymbol{q}^{(n)}\right|^{2} d x=1, \\
\lim _{n \rightarrow \infty} Q\left(F^{(n)}+\nabla \boldsymbol{q}^{(n)} ; Y\right)=\int_{Y}\left(\Perp\left(F^{(n)}+\nabla \boldsymbol{q}^{(n)}\right), F^{(n)}+\nabla \boldsymbol{q}^{(n)}\right) d x=0 .
\end{gathered}
$$

Selecting a subsequence we have

$$
F^{(n)} \rightarrow F=\boldsymbol{a} \otimes \boldsymbol{b}, \quad \boldsymbol{q}^{n} \rightarrow \boldsymbol{q} \quad \text { weakly in } \mathscr{H}_{\#}^{1},
$$

and we obtain from Theorem 3.3(ii), (v) that

$$
\int_{Y}(\mathbb{L}(x)(\boldsymbol{a} \otimes \boldsymbol{b}+\nabla \boldsymbol{q}), \boldsymbol{a} \otimes \boldsymbol{b}+\nabla \boldsymbol{q}) d x=0 .
$$

We assert that $\boldsymbol{a} \otimes \boldsymbol{b} \neq 0$. Otherwise $F^{(n)} \rightarrow 0$ and hence

$$
\int_{Y}\left(\Perp(x) \nabla \boldsymbol{q}^{(n)}, \nabla \boldsymbol{q}^{(n)}\right) d x \rightarrow 0 .
$$

Since $\Lambda_{6}>0$ this implies that $\int_{Y}\left|\nabla \boldsymbol{q}^{(n)}\right|^{2} d x \rightarrow 0$, which contradicts (4.32). From Theorem 3.3 (iv) and (4.33) we deduce (3.28). Finally, (3.27) follows from 
the fact that $q$ minimizes $\mathscr{L}_{1}$, the argument being analogous to the one used to derive (3.26) in Section 4.6.

\subsection{Proof of Proposition 3.6}

The identity $\tilde{\Lambda}_{4}=\tilde{\Lambda}_{5}$ is proved in the same way as $\Lambda_{4}=\Lambda_{5}$; see Theorem 3.3 (i) and Section 4.2. To show (3.30) let $\boldsymbol{q}=\boldsymbol{q}_{1}+\boldsymbol{q}_{2} \in N_{1} \otimes N_{1}^{\perp}$. For $F=\boldsymbol{a} \otimes \boldsymbol{b}$, by the definition of $N_{1}$ and by (4.15), (4.16) we have

$$
\int_{Y}(\mathbb{L}(x)(F+\nabla \boldsymbol{q}), F+\nabla \boldsymbol{q}) d x=\int_{Y}\left(\mathbb{L}(x)\left(F+\nabla \boldsymbol{q}_{2}\right), F+\boldsymbol{q}_{2}\right) d x .
$$

Now (3.30) follows since

$$
\int_{Y}\left|F+\nabla \boldsymbol{q}_{2}\right|^{2} d x=|F|^{2}+\int_{Y}\left|\nabla \boldsymbol{q}_{2}\right|^{2} d x \geqq|F|^{2} .
$$

If $\tilde{\Lambda}_{4}=0$, we can argue as in the proof of Theorem 3.4(iii), see Section 4.7, using (4.18) instead of $A_{6}>0$.

\subsection{Proof of Theorem 3.7 (for stratified materials)}

(i) By Lemma 4.2, $A=\Lambda_{2}$ and thus obviously $\Lambda \leqq \Lambda_{1}^{s}$. To show the opposite inequality it suffices, in view of (4.4), to establish that

$$
\int_{Y}\left(\mathbb{L}\left(x_{N}\right) \nabla v, \nabla v\right) d x \geqq \Lambda_{1}^{s} \int_{Y}|\nabla v|^{2} d x,
$$

whenever $v=e^{i \omega \cdot x} p$, where $p \in \mathscr{H}_{\#}^{1}$ has a finite Fourier series. For any such $p$ one has the (finite) expansion

$$
p\left(x^{\prime}, x_{N}\right)=\sum_{\alpha^{\prime} \in(2 \pi \mathbb{Z})^{N-1}} e^{i \alpha^{\prime} \cdot x^{\prime}} a_{\alpha^{\prime}}\left(x_{N}\right)
$$

with $a_{\alpha^{\prime}} \in \mathscr{H}_{\#}^{1}([0 ; 1])$. Thus, setting $\hat{\alpha}=\left(\alpha^{\prime}, 0\right)$ we have

$$
\begin{aligned}
& \begin{array}{l}
\int_{Y}\left(\amalg\left(x_{N}\right) \nabla \boldsymbol{v}, \nabla \boldsymbol{v}\right) d x \\
=\int_{Y} \sum_{\alpha^{\prime}, \beta^{\prime}} e^{i\left(\alpha^{\prime}-\beta^{\prime}\right) \cdot x}\left(\amalg\left(x_{N}\right)\left(\boldsymbol{a}_{\alpha^{\prime}} \otimes i(\hat{\alpha}+\omega)+\frac{\partial}{\partial x_{N}} \boldsymbol{a}_{\alpha^{\prime}} \otimes \boldsymbol{e}_{N}\right),\right. \\
\left.\boldsymbol{a}_{\beta^{\prime}} \otimes i(\hat{\beta}+\omega)+\frac{\partial}{\partial x_{N}} \boldsymbol{a}_{\beta^{\prime}} \otimes \boldsymbol{e}_{N}\right) d x .
\end{array} \\
& \text { Now }
\end{aligned}
$$

and hence

$$
\int_{\left[0,11^{N-1}\right.} \sum_{\alpha^{\prime}, \beta^{\prime}} e^{i\left(\alpha^{\prime}-\beta^{\prime}\right) \cdot x^{\prime}} d x_{1} \ldots d x_{N-1}=\delta_{\alpha^{\prime} \beta^{\prime}}
$$

$$
\begin{aligned}
\int_{Y}\left(\mathbb{L}\left(x_{N}\right) \nabla \boldsymbol{v}, \nabla \boldsymbol{v}\right) d x & =\sum_{\alpha^{\prime}} \int_{Y}\left(\mathbb{L}\left(x_{N}\right) \nabla\left(e^{i(\hat{\alpha}+\omega) \cdot x} a_{\alpha^{\prime}}\right), \nabla\left(e^{i(\hat{\alpha}+\omega) \cdot x} a_{\alpha^{\prime}}\right)\right) d x \\
& \geqq \sum_{\alpha^{\prime}} \Lambda_{1}^{s} \int_{Y}\left|\nabla\left(e^{i(\hat{\alpha}+\omega) \cdot x} a_{\alpha^{\prime}}\right)\right|^{2} d x=\Lambda_{1}^{s} \int|\nabla \boldsymbol{v}|^{2} d x,
\end{aligned}
$$

and (i) is proved. 
(ii), (iii), (iv). These are proved as Theorem 3.3(i), Theorem 3.1(i), (ii) and Theorem 3.4(i), respectively, except for the assertion that $\mathbb{M}^{s}=\mathbb{M}_{H}$. We sketch the proof of this and assume for convenience that $H=(1, \ldots, 1)$, so $Z=Y$. Recall that the infimum in (3.37) may be taken over the complexified

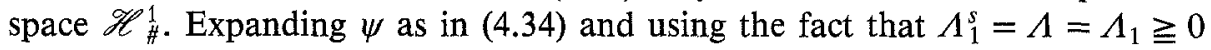
one finds by virtue of the orthogonality relations (4.35) that

$$
\begin{aligned}
& \int_{Y}\left(\mathbb{L}\left(x_{N}\right)(G+\nabla \psi), G+\nabla \psi\right) d x \\
& =\int_{0}^{1}\left(\mathbb{L}\left(x_{N}\right)\left(G+\nabla a_{0}\right), G+\nabla a_{0}\right) d x_{N}+\int_{Y}\left(\mathbb{L}\left(x_{N}\right)\left(\nabla\left(\psi-a_{0}\right)\right), \nabla\left(\psi-a_{0}\right)\right) d x \\
& \geqq\left(\mathbb{M}^{s} G, G\right) .
\end{aligned}
$$

Thus $\left(M_{H} G, G\right) \geqq\left(M^{s} G, G\right)$ and the reverse inequality is obvious.

(v), (vi). These are shown as Theorem 3.4(ii), (iii), respectively.

(vii) The inequality $\Lambda_{6}^{s} \leqq \alpha_{\Perp}^{s}$ is proved analogously to $\Lambda_{6} \leqq \alpha_{\Perp}$; see Section 4.2. The inequality $\alpha_{\mathbb{L}} \leqq \alpha_{\mathbb{S}}^{\mathrm{s}}$ is obvious. On the other hand, for $\boldsymbol{q}\left(x_{N}\right) \epsilon$ $H_{\#}^{1}([0,1])$ (extended to $\mathbb{R}^{N}$ ) one has $\nabla \boldsymbol{q}=\frac{d \boldsymbol{q}}{d x_{N}} \otimes \boldsymbol{e}_{N}$, so that

$$
\begin{aligned}
\int_{Y}\left(\mathbb{L}\left(x_{N}\right) \nabla \boldsymbol{q}, \nabla \boldsymbol{q}\right) d x & =\int_{Y}\left(\mathbb{L}\left(x_{N}\right) \frac{d \boldsymbol{q}}{d x_{N}} \otimes \boldsymbol{e}_{N}, \frac{d \boldsymbol{q}}{d x_{N}} \otimes \boldsymbol{e}_{N}\right) d x \\
& \geqq \int_{0}^{1} \alpha_{\mathbb{U}}^{s}\left|\frac{d \boldsymbol{q}}{d x_{N}}\right|^{2} d x_{N}=\alpha_{\mathbb{L}}^{s} \int_{0}^{1}\left|\frac{d \boldsymbol{q}}{d x_{N}}\right|^{2} d x_{N}=\alpha_{\mathbb{L}}^{s} \int_{Y}|\nabla \boldsymbol{q}|^{2} d x .
\end{aligned}
$$

Hence $\Lambda_{6}^{s} \geqq \alpha_{\mathbb{L}}^{s}$, so that $\Lambda_{6}^{s}=\alpha_{\mathbb{L}}^{s}$.

\section{Nonlinear Homogenization and Loss of Strong Ellipticity}

\subsection{General remarks}

Here we take up the problem of analyzing the behavior of the nonlinear homogenized energy density $\bar{W}$. A formal calculation suggests that the second derivatives $\frac{\partial^{2} \bar{W}}{\partial F^{2}}\left(F_{0}\right)$ are given by the homogenization of linearized problems of the type discussed in the two previous sections.

Of particular interest is the question whether $\bar{W}$ is strongly elliptic, i.e., whether

$$
\frac{\partial^{2} \bar{W}}{\partial F^{2}}\left(F_{0}\right)(\boldsymbol{a} \otimes \boldsymbol{b}, \boldsymbol{a} \otimes \boldsymbol{b}) \geqq c|\boldsymbol{a} \otimes \boldsymbol{b}|^{2}, \quad c>0, \forall \boldsymbol{a}, \boldsymbol{b} \in \mathbb{R}^{N} .
$$

Failure of the strong ellipticity condition would allow for the possibility of shear band instabilities in the homogenized material. 
It should be noted that by general results on $\Gamma$-convergence (cf. Section 2 ), $\bar{W}$ always satisfies $\frac{\partial^{2} \bar{W}}{\partial F^{2}}\left(F_{0}\right)(\boldsymbol{a} \otimes \boldsymbol{b}, \boldsymbol{a} \otimes \boldsymbol{b}) \geqq 0$ or, more precisely, $t \mapsto \bar{W}\left(F_{0}+t \boldsymbol{a} \otimes \boldsymbol{b}\right)$ always is convex. The question is whether one has strict inequality or strict convexity.

To avoid technical difficulties we do not pursue here the question whether $\bar{W}$ is indeed twice differentiable for general integrands $W(x, F)$ and mainly confine ourselves to second-order asymptotic expansions of $\bar{W}\left(F_{0}+t \boldsymbol{a} \otimes \boldsymbol{b}\right)$ as $t \rightarrow 0$.

In Section 5.4 we show, however, how more complete results can be obtained for integrands $W(x, F)$ which are convex in $F$ (which is unfortunately an unrealistic assumption in elasticity).

\subsection{Main results}

We consider integrands $W(x, F)$ satisfying (2.1)-(2.3) (as well as additional assumptions stated below) and recall the definitions $\hat{W}^{k}$ and $\bar{W}$ in (2.6) and (2.7). Our first result depends on the following hypothesis.

(H1) There exists $t_{0}>0$ such that for all $0 \leqq t \leqq t_{0}$ and all $H=a \otimes b$ with $|H|=1$,

$$
\bar{W}\left(F_{0}+t H\right)=\hat{W}^{1}\left(F_{0}+t H\right)
$$

and there exist minimizers $\varphi_{F_{0}+t H} \in W_{\sharp}^{1, \infty}$, i.e.,

$$
\bar{W}\left(F_{0}+t H\right)=\int_{Y} W\left(x, F_{0}+t H+\nabla \varphi_{F_{0}+t H}(x)\right) d x,
$$

satisfying $\int_{Y} \varphi_{F_{0}+t H}=0$ and

$$
\left\|\varphi_{F_{0}+t H}-\varphi_{F_{0}}\right\|_{W^{1, \infty}} \leqq r(t),
$$

where $r(t) \rightarrow 0$ as $t \rightarrow 0$.

Equation (5.1) states that near $F_{0}$ the homogenized energy is given by the solution of an auxiliary problem on only one periodic cell, while (5.3) requires that no discontinuous bifurcation of minimizers occurs. The exclusion of discontinuous bifurcation seems plausible from a physical point of view and such an assumption is implicit in many numerical schemes to compute minimizers (e.g., by path-following techniques). There are, however, no rigorous mathematical results which would allow one to deduce (5.3) from reasonable assumptions on $W$. Indeed the current theory of nonlinear elliptic systems even leaves open the question as to whether $\varphi_{F_{0}+t H}$ is bounded in $W_{\#}^{1, \infty}$.

The linearized elasticity tensor at $F_{0}$ is defined by

$$
\uplus_{F_{0}}(x)=\frac{\partial^{2} W}{\partial F^{2}}\left(x, F_{0}+\nabla \varphi_{F_{0}}(x)\right)
$$


Corresponding to $\mathbb{L}_{F_{0}}(x)$ we can define as in Section 3

$$
\Lambda\left(F_{0}\right)=\inf \left\{\frac{\int_{\mathbb{R}^{N}}\left(\uplus_{F_{0}}(x) \nabla \boldsymbol{u}, \nabla \boldsymbol{u}\right) d x}{\int_{\mathbb{R}^{N}}|\nabla \boldsymbol{u}|^{2} d x} \mid \boldsymbol{u} \in \mathscr{D}\left(\mathbb{R}^{N}, \mathbb{R}^{N}\right)\right\},
$$

cf. (3.11), and similarly define $\Lambda_{4}\left(F_{0}\right), \Lambda_{6}\left(F_{0}\right), \alpha_{\llbracket}\left(F_{0}\right)$; cf. (3.20), (3.22), (3.7).

If $A_{4}\left(F_{0}\right) \geqq 0$, then in view of Theorem $3.3(\mathrm{v})$ we can define the 4-tensor $M_{F_{0}}$ by the analogue of (3.18):

$$
\left(\mathbb{M}_{F_{0}} G, G\right)=\inf \left\{\int_{Y}\left(\mathbb{L}_{F_{0}}(x)(G+\nabla \boldsymbol{q}), G+\nabla \boldsymbol{q}\right) d x \mid \boldsymbol{q} \in H_{\#}^{1}\right\},
$$

and $M_{F_{0}}$ satisfies

$$
\left(M_{F_{0}} a \otimes b, a \otimes b\right) \geqq \Lambda_{4}\left(F_{0}\right)|\boldsymbol{a} \otimes \boldsymbol{b}|^{2} \quad \text { for all } \boldsymbol{a}, \boldsymbol{b} \in \mathbb{R}^{N} .
$$

Finally the average stress tensor is characterized by

$$
\bar{\sigma}\left(F_{0}\right)=\int_{Y} \frac{\partial W}{\partial F}\left(x, F_{0}+\nabla \varphi_{F_{0}}(x)\right) d x .
$$

We can now state the main result of this section.

Theorem 5.1. Assume that $W(x, F)$ satisfies (2.1), (2.2), (2.3), (H 1) and moreover

$$
\begin{gathered}
W(x, \cdot) \text { is in } C^{3}, \\
\left|\frac{\partial^{3} W}{\partial F^{3}}(x, F)\right| \leqq h(F)
\end{gathered}
$$

where $h$ is locally bounded.

(i) If $\Lambda_{4}\left(F_{0}\right)>0$, then for all $H=\boldsymbol{a} \otimes \boldsymbol{b}$, with $|H|=1$,

$$
\bar{W}\left(F_{0}+t H\right)=\bar{W}\left(F_{0}\right)+\bar{\sigma}\left(F_{0}\right) H t+\frac{1}{2}\left(\mathbb{M}_{F_{0}} H, H\right) t^{2}+o\left(t^{2}\right) \quad \text { as } t \rightarrow 0 \text {. }
$$

(ii) If $\Lambda_{4}\left(F_{0}\right)=0, \Lambda_{6}\left(F_{0}\right)>0$, then there exists $H=a \otimes b$, with $|H|=1$ such that

$$
\bar{W}\left(F_{0}+t H\right)=\bar{W}\left(F_{0}\right)+\bar{\sigma}\left(F_{0}\right) H t+o\left(t^{2}\right) \quad \text { as } t \rightarrow 0 .
$$

Remark. Note that the result in (ii) implies that $\bar{W}$ loses uniform rank-1 convexity in the direction $\boldsymbol{a} \otimes \boldsymbol{b}$. Moreover (ii) remains valid if (H1) is replaced by the weaker hypothesis $\bar{W}\left(F_{0}\right)=\hat{W}^{1}\left(F_{0}\right)$.

Proof. (i) Let $H=a \otimes b$ with $|H|=1$ be fixed. Set

$$
V(t, H)=\bar{W}\left(F_{0}+t H\right)-\bar{W}\left(F_{0}\right)-\bar{\sigma}\left(F_{0}\right) t H-\frac{1}{2}\left(\mathbb{M}_{F_{0}} H, H\right) t^{2} .
$$


From (5.1), the Euler-Lagrange equation for $\varphi_{F_{0}}$ and the Taylor expansion of $W(x, F)$ at $F_{0}+\nabla \varphi_{F_{0}}$ we obtain for every $\psi \in W_{\#}^{1, \infty}$ that

$\bar{W}\left(F_{0}+t H\right)$

$\leqq \int_{Y} W\left(x, F_{0}+t H+\nabla \varphi_{F_{0}}+\nabla \psi\right) d x$

$=\bar{W}\left(F_{0}\right)+\bar{\sigma}\left(F_{0}\right) H t$

$+\int_{Y} \int_{0}^{1}(1-\eta)\left[\left(\frac{\partial^{2} W}{\partial F^{2}}\left(x, F_{0}+\nabla \varphi_{F_{0}}+\eta(t H+\nabla \psi)\right)(t H+\nabla \psi), t H+\nabla \psi\right)\right] d \eta d x$

with equality for $\psi=\varphi_{F_{0}+t H}-\varphi_{F_{0}}$. Step 1
that $\left(\liminf _{t \rightarrow 0} t^{-2} V(t) \geqq 0\right)$. Set $\psi=\varphi_{F_{0}+t H}-\varphi_{F_{0}}$. From (5.13) we deduce

$$
\begin{aligned}
\bar{W}\left(F_{0}+t H\right) & -\bar{W}\left(F_{0}\right)-\bar{\sigma}\left(F_{0}\right) H t \\
= & \frac{1}{2} \int_{Y}\left(\mathbb{F}_{F_{0}}(x)(t H+\nabla \psi), t H+\nabla \psi\right) d x \\
& +\int_{Y} \int_{0}^{1}(1-\eta)\left(\left[\tilde{\mathbb{L}}-\Perp_{F_{0}}\right](x)(t H+\nabla \psi), t H+\nabla \psi\right) d \eta d x,
\end{aligned}
$$

where we have set

$$
\tilde{\mathbb{L}}(x)=\frac{\partial^{2} W}{\partial F^{2}}\left(x, F_{0}+\nabla \varphi_{F_{0}}+\eta(t H+\nabla \psi)\right) .
$$

From (5.9) and (5.10) it follows that

$\left|\left(\left[\tilde{\mathbb{I}}-\mathbb{U}_{F_{0}}\right](x)(t H+\nabla \psi), t H+\nabla \psi\right)\right| \leqq c_{1}\left(t+\|\psi\|_{W^{1, \infty}}\right)|t H+\nabla \psi|^{2}$.

Using $\Lambda_{4}\left(F_{0}\right)>0$ and (5.3) we have for $t$ small enough that

$$
\begin{aligned}
& \bar{W}\left(F_{0}+t H\right)-\bar{W}\left(F_{0}\right)-\bar{\sigma}\left(F_{0}\right) H t \\
& \geqq \frac{1}{2}\left[1-\frac{c_{1}}{\Lambda_{4}\left(F_{0}\right)}(t+r(t))\right] \int_{Y}\left(\mathbb{F}_{F_{0}}(x)(t H+\nabla \psi), t H+\nabla \psi\right) d x .
\end{aligned}
$$

Thus from (5.6), (5.7), we obtain

$$
\bar{W}\left(F_{0}+t H\right)-\bar{W}\left(F_{0}\right)-\bar{\sigma}\left(F_{0}\right) H t \geqq \frac{1}{2}\left[1-\frac{c_{1}}{\Lambda_{4}\left(F_{0}\right)}(t+r(t))\right]\left(\mathbb{M}_{F_{0}} H, H\right) t^{2} .
$$

In conclusion, for $t$ small enough we find that

$$
\begin{aligned}
\bar{W}\left(F_{0}+t H\right)-\bar{W}\left(F_{0}\right)-\bar{\sigma}\left(F_{0}\right) & H t-\frac{1}{2}\left(M_{F_{0}} H, H\right) t^{2} \\
& \geqq-\frac{c_{1}}{2 \Lambda_{4}\left(F_{0}\right)}\left(\mathbb{M}_{F_{0}} H, H\right)(t+r(t)) t^{2},
\end{aligned}
$$


and finally

$$
\lim _{t \rightarrow 0} t^{-2} V(t) \geqq 0 .
$$

Step 2. $\left(\lim _{t \rightarrow 0} t^{-2} V(t) \leqq 0\right)$. Fix $\delta>0$ and choose $v \in W_{\#}^{1, \infty}$ such that

$$
\int_{Y}\left(\mathbb{L}_{F_{0}}(x)(H+\nabla v, H+\nabla v)\right) d x \leqq\left(\mathbb{M}_{F_{0}} H, H\right)+\delta .
$$

From (5.13) with $\psi=t v$ we obtain

$t^{-2}\left\{\bar{W}\left(F_{0}+t H\right)-\bar{W}\left(F_{0}\right)-\bar{\sigma}\left(F_{0}\right) H t\right\}$

$\leqq \int_{0}^{1} \int_{Y}(1-\eta)\left[\left(\frac{\partial^{2} W}{\partial F^{2}}\left(x, F_{0}+\nabla \varphi_{F_{0}}+\eta t(H+\nabla v)\right)(H+\nabla v), H+\nabla v\right)\right] d x d \eta$.

As $t \rightarrow 0$, the right-hand side converges to

$$
\int_{Y}\left(\mathbb{L}_{F_{0}}(x)(H+\nabla v), H+\nabla v\right) d x \leqq\left(\mathbb{M}_{F_{0}} H, H\right)+\delta,
$$

and thus

$$
\limsup _{t \rightarrow 0} V(t, H) \leqq \delta .
$$

Since $\delta>0$ is arbitrary, statement (i) of Theorem 5.1 follows from (5.18) and (5.21).

(ii) By Theorem 3.4(iii) we may choose $H=\boldsymbol{a} \otimes \boldsymbol{b} \neq 0$ such that $\mathbb{M}_{F_{0}}(H, H)=0$. Arguing as in Step 2 with $\bar{W}$ replaced by $\hat{W}^{1}$, we find

$$
\limsup _{t \rightarrow 0}\left(t^{-2} \hat{W}^{1}\left(F_{0}+t H\right)-\hat{W}^{1}\left(F_{0}\right)-\bar{\sigma}_{F_{0}} H t\right) \leqq 0 .
$$

The result now follows from the assumption that $\hat{W}^{1}\left(F_{0}\right)=\bar{W}\left(F_{0}\right)$, the fact that $t \rightarrow \bar{W}\left(F_{0}+t H\right)$ is convex and the following proposition. Note in particular that $(\mathrm{H} 1)$ is not required to prove (ii).

Proposition 5.2. Assume that $g: \mathbb{R} \rightarrow \mathbb{R}$ satisfies

$$
\limsup _{t \rightarrow 0} t^{-2}(g(t)-g(0)-a t) \leqq 0
$$

for some $a \in \mathbb{R}$ and that $f: \mathbb{R} \rightarrow \mathbb{R}$ is a convex function satisfying $f(0)=g(0)$ and $f \leqq g$. Then

$$
\lim _{t \rightarrow 0} t^{-2}(f(t)-f(0)-a t)=0 .
$$

Proof. Since $f$ is convex there is a $b \in \mathbb{R}$ such that $f(t)-f(0)-b t \geqq 0$. In particular,

$$
\liminf _{t \rightarrow 0} t^{-2}(f(t)-f(0)-b t) \geqq 0 .
$$

Subtracting (5.23) from (5.22) and using $f \leqq g$ one finds

$$
\limsup _{t \rightarrow 0} \frac{1}{t}(b-a) \leqq \limsup _{t \rightarrow 0} \frac{1}{t^{2}}(g(t)-f(t)+(b-a) t) \leqq 0 .
$$

Hence $a=b$. Now the assertion follows from (5.22) and (5.23) since $f \leqq g$. 


\subsection{A weakened hypothesis}

Assumption (H1) can be weakened a little when $\Lambda\left(F_{0}\right)>0$. More precisely, we assume

(H2) $\bar{W}\left(F_{0}\right)=\hat{W}^{1}\left(F_{0}\right)$, and there exists a corresponding minimizer $\varphi_{F_{0}} \in W_{\#}^{1, \infty}$ satisfying $\int_{Y} \varphi_{F_{0}}(x) d x=0$. Moreover, there exists $t_{0}>0$ such that for all $0 \leqq t \leqq t_{0}$, all $H=\boldsymbol{a} \otimes \boldsymbol{b}$ with $|H|=1$ and all $k \in \mathbb{N}^{*}$ there exist minimizers $\varphi_{F_{0}+t H}^{k} \in W_{k \#}^{1, \infty}$ satisfying

$$
\begin{gathered}
\int_{k Y} \varphi_{F_{0}+t H}^{k}(x) d x=0, \\
\hat{W}^{k}\left(F_{0}+t H\right)=\min _{\varphi \in W_{k \neq}^{1, p}} \frac{1}{k^{N}} \int_{k Y} W\left(x, F_{0}+t H+\nabla \varphi\right) d x \\
=\frac{1}{k^{N}} \int_{k Y} W\left(x, F_{0}+t H+\nabla \varphi_{F_{0}+t H}^{k}\right) d x, \\
\left\|\varphi_{F_{0}+t H}^{k}-\varphi_{F_{0}}^{k}\right\|_{W_{k t}^{1, \infty}}^{1} \leqq r(t)
\end{gathered}
$$

where $r(t) \rightarrow 0$ as $t \rightarrow 0$ and $\varphi_{F_{0}}^{k} \in W_{k t}^{1, \infty}$ denotes the periodic exension of $\varphi_{F_{0}} \in W_{\ddot{\#}}^{1, \infty}$.

Proposition 5.3. Assume that $W(x, F)$ satisfies (2.1), (2.2), (2.3), (5.9), (5.10). Moreover, assume that $(\mathrm{H} 2)$ holds and that $\Lambda\left(F_{0}\right)>0$. Then there exists $\delta_{0}>0$ such that for all $0 \leqq t \leqq \delta_{0}$, all $H=\boldsymbol{a} \otimes \boldsymbol{b}$ with $|H|=1$ and all $k \in \mathbb{N}^{*}$ the minimizers $\varphi_{F_{0}+t H}^{k}$ appearing in (H2) satisfy

$$
\varphi_{F_{0}+t H}^{k}=\varphi_{F_{0}+t H}^{1} .
$$

In particular, $\bar{W}\left(F_{0}+t H\right)=\hat{W}^{1}\left(F_{0}+t H\right)$ and (H1) is satisfied. Moreover, $\varphi_{F_{0}+t H}^{k}$ is locally the unique minimizer (in $\left.W_{k \#}^{1, \infty}\right)$ of

$$
\varphi \mapsto \frac{1}{k^{N}} \int_{k Y} W\left(x, F_{0}+t H+\nabla \varphi\right) d x,
$$

i.e., there exists a $\delta_{1}>0$ such that whenever $\varphi_{F_{0}+t H}^{k}+\psi$ with $\|\psi\|_{W_{k+1}^{1, \infty}}<\delta_{1}$ is a minimizer (or merely a weak solution of the Euler-Lagrange equation), then $\nabla \psi \equiv 0$.

Remark. Under the continuity hypothesis stated in ( $\mathrm{H} 2)$, Proposition 5.3 allows us to apply the conclusions of Theorem 5.1 along a rank-1 path of matrices $F_{s}$ (corresponding, e.g., to increasing uniaxial compression). More specifically, if $\varphi_{F_{0}}^{1}=\varphi_{F_{0}}^{k}$, then $\varphi_{F_{s}}^{1}=\varphi_{F_{s}}^{k}$ and the expansion (5.11) holds around $F_{s}$ as long as $\Lambda\left(\mathbb{F}_{F_{s}}\right)>0$. If $\Lambda\left(\mathbb{L}_{F_{s^{*}}}\right)=0$, then two cases may occur: Either $\Lambda_{4}\left(\mathbb{L}_{F_{s}}\right)=0$, in which case the homogenized energy loses uniform rank-1 convexity (Theorem 5.1(ii)) and the linearized equation admits a shear-band type solution (Theorem 3.4(iii)), or $A_{4}\left(\uplus_{F_{s t}}\right)>0$, in which case the linearized equation admits a Bloch-wave solution (Theorem 3.4(ii)). In this case it is plausible that $\bar{W}$ retains uniform rank-1 convexity at $F_{0}$ but we are only able to 
prove this under the more restrictive hypothesis (H1) (with $F_{0}$ replaced by $\left.F_{s^{*}}\right)$. This confirms the statement in [TM 85] that long-wavelength instabilities $\left(\Lambda_{5}=\Lambda_{4}=0\right)$ correspond to loss of (strong) ellipticity for the homogenized material.

Proof. It suffices to prove the uniqueness statement since the periodic extension of $\varphi_{F_{0}+t H}^{1}$ is a weak solution of the Euler-Lagrange equation, i.e.,

Indeed,

$$
\frac{1}{k^{N}} \int_{k Y} \frac{\partial W}{\partial F}\left(x, F_{0}+t H+\nabla \varphi_{F_{0}+t H}^{1}\right) \nabla \eta d x=0 \quad \forall \eta \in W_{k \#}^{1, p} .
$$

$\frac{1}{k^{N}} \int_{k Y} \frac{\partial W}{\partial F}\left(x, F_{0}+t H+\nabla \varphi_{F_{0}+t H}^{1}\right) \nabla \eta d x=\int_{Y} \frac{\partial W}{\partial F}\left(x, F_{0}+t H+\nabla \varphi_{F_{0}+t H}^{1}\right) \nabla \tilde{\eta} d x$, where $\tilde{\eta}(x)=\sum_{z \in\{0, \ldots, k-1\}^{N}} \eta(x+z) \in W_{\#}^{1, p}$ and the second integral is zero since $\varphi_{F_{0}+t H}^{1}$ is a minimizer in $W_{\#}^{1, p}$.

So assume that $\varphi=\varphi_{F_{0}+t H}^{k}+\psi \in W_{k \neq t}^{1, p}$ is a weak solution of the EulerLagrange equation, i.e.,

$$
\frac{1}{k^{N}} \int_{k Y} \frac{\partial W}{\partial F}\left(x, F_{0}+t H+\nabla \varphi\right) \nabla \eta d x=0 \quad \forall \eta \in W_{k \#}^{1, p}
$$

and $\|\nabla \psi\|_{W_{k \neq}^{1, \infty}<}<\delta_{1}$, with $\delta_{1}$ to be chosen later. By the minimizing property of $\varphi_{F_{0}+t H}^{k}$ and by the Taylor expansion around $F_{0}+t H+\nabla \varphi(x)$ we find

$$
0 \geqq \int_{k Y}\left(\bigsqcup_{F_{0}}(x) \nabla \psi, \nabla \psi\right) d x+2 \int_{k Y} \int_{0}^{1}(1-\eta)\left(\left[\tilde{\mathbb{L}}-\mathbb{U}_{F_{0}}\right] \nabla \psi, \nabla \psi\right) d \eta d x,
$$

where

Since

$$
\tilde{\mathbb{L}}=\frac{\partial^{2} W}{\partial F^{2}}\left(x, F_{0}+t H+\nabla \varphi-\eta \nabla \psi\right) .
$$

$$
\begin{aligned}
\left|F_{0}+t H+\nabla \varphi-\eta \nabla \psi-\left(F_{0}+\nabla \varphi_{F_{0}}^{k}\right)\right| & =\left|t H+\nabla\left(\varphi_{F_{0}+t H}^{k}-\varphi_{F_{0}}^{k}\right)+(1-\eta) \nabla \psi\right| \\
& \leqq t+\left|\nabla\left(\varphi_{F_{0}+t H}^{k}-\varphi_{F_{0}}^{k}\right)\right|+|\nabla \psi|,
\end{aligned}
$$

it follows from (5.24) that we can choose $\delta_{0}>0$ such that

$$
\left|F_{0}+t H+\nabla \varphi-\eta \nabla \psi-\left(F_{0}+\nabla \varphi_{F_{0}}^{k}\right)\right| \leqq 2 \delta_{1} \quad \text { for all } 0 \leqq t \leqq \delta_{0} .
$$

Therefore by (5.9), (5.10) we have

$$
\left\|\tilde{\mathbb{L}}-\mathbb{H}_{F_{0}}\right\|_{\infty} \leqq C \delta_{1} .
$$

In view of Lemma 4.2 we then deduce from (5.27) that

Choose $\delta_{1}=\frac{\Lambda\left(F_{0}\right)}{2 C}$; then $\nabla \psi \equiv 0$ and the proof is finished.

$$
\begin{aligned}
& 0 \geqq\left(\Lambda\left(F_{0}\right)-C \delta_{1}\right) \int_{k Y}|\nabla \psi|^{2} d x \\
& \text { then } \nabla \psi \equiv 0 \text { and the proof is finished. }
\end{aligned}
$$




\subsection{Convex integrands}

We complete this section by showing how the relation between homogenization and linearization can be made rigorous for convex integrands without the use of implicit hypotheses like (H1) or (H2) above. To avoid technical difficulties we consider only strictly convex integrands with quadratic growth. Specifically we assume that

$$
\begin{gathered}
W(x+z, F)=W(x, F) \quad \text { for } z \in \mathbb{Z}^{N}, \\
W(x, \cdot) \text { is convex and in } C^{2}, \\
C|F|^{2} \leqq W(x, F) \leqq C\left(1+|F|^{2}\right), \\
\quad\left|\frac{\partial W}{\partial F}(x, F)\right| \leqq C(1+|F|), \\
c|G|^{2} \leqq \frac{\partial^{2} W}{\partial F^{2}}(x, F)(G, G) \leqq C|G|^{2},
\end{gathered}
$$

where $c>0$. MARCELLINI [Ma 78] showed that the homogenized energy density is given by

$$
\hat{W}(F)=\hat{W}^{1}(F)=\min _{y \in H_{\sharp}^{1}} \int_{Y} W(x, F+\nabla y) d x .
$$

The minimizer corresponding to $F$ is unique since $W$ is strictly convex and is denoted by $y_{F}$. As before,

$$
\mathbb{1}_{F}(x)=\frac{\partial^{2} W}{\partial F^{2}}\left(x, F+\nabla y_{F}(x)\right)
$$

is the linearized elastic tensor and $M_{F}$ its homogenization. Let

$$
\bar{\sigma}_{F}=\int_{Y} \frac{\partial W}{\partial F}\left(x, F+\nabla y_{F}\right) d x .
$$

The main result of this section states that homogenization and linearization commute.

Theorem 5.4. Let (5.28)-(5.32) hold. Then the homogenized energy density $\hat{W}$ is in $C^{2}$ and

$$
\frac{\partial^{2} \hat{W}}{\partial F^{2}}(G, G)=\left(M_{F} G, G\right)
$$

Remark. Similar results have been obtained by FrancFort \& Murat ([FM 91]) and for scalar functions by AтTOUCH [A 84].

We shall use the following result.

Proposition 5.5. Let $\bigsqcup^{j}$ be a sequence of measurable, symmetric 4-tensors satisfying

$$
c|G|^{2} \leqq\left(\mathbb{H}^{j}(x) G, G\right) \leqq C|G|^{2}, \quad c>0
$$


for a.e. $x \in Y$ and for all $N \times N$ matrices $G$. Assume that $\mathbb{U}^{j} \rightarrow \mathbb{L}$ a.e. Let $\psi_{G}^{j}$ be the minimizers in $H_{\#}^{1}$ of

$$
\int_{Y}\left(\mathbb{L}^{j}(x)(G+\nabla \psi), G+\nabla \psi\right) d x
$$

subject to $\int_{Y} \psi_{G}^{j} d x=0$. Then $\psi_{G}^{j} \rightarrow \psi_{G}$ strongly in $H_{\#}^{1}$ where $\psi_{G}$ is the minimizer of

$$
\int_{Y}(\mathbb{L}(x)(G+\nabla \psi), G+\nabla \psi) d x .
$$

In particular,

$$
\int_{Y}\left(\mathbb{L}^{j}(x)\left(G+\nabla \psi_{G}^{j}\right), G+\nabla \psi_{G}^{j}\right) d x \rightarrow \int_{Y}\left(\mathbb{L}(x)\left(G+\nabla \psi_{G}\right), G+\nabla \psi_{G}\right) d x .
$$

The proof of the proposition is standard and is deferred to the end of this section.

Proof of Theorem 5.4. Fix $N \times N$ matrices $F, G,|G|=1$. Set $h(t, G) \stackrel{\text { def }}{=}$ $t^{-2}\left(\hat{W}(F+t G)-\hat{W}(F)-\bar{\sigma}_{F} t G\right)$. A Taylor expansion in connection with the Euler-Lagrange equation for $y_{F}$ gives

$h(t, G)=\min _{\psi \in H_{\sharp}^{1}} \int_{Y} \int_{0}^{1}(1-s) \frac{\partial^{2} W}{\partial F^{2}}\left(F+\nabla y_{F}+s t(G+\nabla \psi)\right)(G+\nabla \psi, G+\nabla \psi) d s d x$.

Step 1 (lower bound). Choose a sequence $t_{j} \rightarrow 0$ such that $\liminf _{t \rightarrow 0} h(t, G)=$ $\lim _{j \rightarrow \infty} h\left(t_{j}, G\right)$. Let $\psi_{j}$ be the minimizer corresponding to $h\left(t_{j}, G\right)$ (see (5.35)) with $\int_{Y} \psi_{j} d x=0$. In view of (5.32) one has $\left\|\psi_{j}\right\|_{H^{1}} \leqq C$. Set

$$
\mathbb{U}^{s, j}(x)=\frac{\partial^{2} W}{\partial F^{2}}\left(x, F+\nabla y_{F}(x)+s t_{j}\left(G+\nabla \psi_{j}(x)\right)\right),
$$

let $\eta^{s, j}$ be the minimizer (in $H_{\sharp}^{1}$ ) of

$$
\int_{Y}\left(\mathbb{L}^{s, j}(x)(G+\nabla \psi), G+\nabla \psi\right) d x
$$

(with $\int_{Y} \eta^{s, j} d x=0$ ) and let $M^{s, j}$ be the corresponding minimum value. Note that

$$
h\left(t_{j}, G\right) \geqq \frac{1}{2} \int_{0}^{1} M^{s, j} d s .
$$

Again by (5.32), observe that $\left|M^{s, j}\right| \leqq C$. Moreover, $t_{j} \nabla \psi_{j} \rightarrow 0$ in $L^{2}$ and hence a.e. (for a subsequence). Thus $\mathbb{L}^{j, s} \rightarrow \mathbb{U}_{F}=\frac{\partial^{2} W}{\partial F^{2}}\left(F+\nabla y_{F}\right)$ a.e. for all $s$ as $j \rightarrow \infty$. By Proposition 5.5, $\eta^{s, j} \rightarrow \eta$ in $H_{\#}^{1}$ and $\eta$ is the minimizer of

$$
\int_{Y}\left(\mathbb{L}_{F}(x)(G+\nabla \psi), G+\nabla \psi\right) d x .
$$


It follows that $M^{s, j} \rightarrow\left(M_{F} G, G\right)$ and therefore (by Fatou's lemma)

$$
\liminf _{t \rightarrow 0} h(t, G)=\liminf _{j \rightarrow \infty} h\left(t_{j}, G\right) \geqq \frac{1}{2}\left(M_{F} G, G\right) .
$$

Step 2 (upper bound). Let $\psi_{G}$ be the minimizer of (5.33). Setting $\psi=\psi_{G}$ in (5.35) one finds

$$
\limsup _{t \rightarrow 0} h(t, G) \leqq \frac{1}{2} \int_{Y}\left(\mathbb{H}_{F}(x)\left(G+\nabla \psi_{G}\right), G+\nabla \psi_{G}\right) d x=\frac{1}{2}\left(\mathbb{M}_{F} G, G\right),
$$

since the term $\frac{\partial^{2} W}{\partial F^{2}}\left(F+\nabla y_{F}+s t\left(G+\nabla \psi_{G}\right)\right)$ converges boundedly a.e. to $\Perp_{F}$ as $t \rightarrow 0$.

Step 3 (continuity of $F \mapsto \bar{\sigma}_{F}, F \mapsto M_{F}$ ). Let $\psi_{t}$ be the minimizer in (5.35). Then $\varphi_{F+t G}=\varphi_{F}+t \psi_{t}$. Since $\left\|\psi_{t}\right\|_{H^{1}} \leqq C$ (see above) with $C$ independent of $G$ (since $|G|=1$ ) one has

$$
\sup _{|G|=1}\left\|\varphi_{F+t G}-\varphi_{F}\right\| \leqq C(F) t .
$$

It follows that $F \mapsto \bar{\sigma}_{F}$ is continuous. Moreover, one finds that $\mathbb{U}_{F_{j}} \rightarrow \mathbb{U}_{F}$ boundedly a.e. whenever $F_{j} \rightarrow F$. The continuity of $F \mapsto M_{F}$ now follows from Proposition 5.4.

Step 4 (differentiability). From Steps $1-3$ and Proposition 5.6 below it follows that $\hat{W}$ is in $C^{2}$ and $\frac{\partial^{2} \hat{W}}{\partial F^{2}}=M_{F}$.

Proposition 5.6. Let $\mathscr{U}$ be an open subset of $\mathbb{R}^{p}$, let $F: \mathscr{U} \rightarrow \mathbb{R}$ be continuous and let $g$ and $h$ be continuous as linear and bilinear forms on $\mathbb{R}^{p}$, respectively. Assume that for all $x \in \mathscr{U}, y \in \mathbb{R}^{p}$,

$$
\lim _{t \rightarrow 0} t^{-2}\left(f(x+t y)-f(x)-\operatorname{tg}(x) y-\frac{1}{2} t^{2}(h(x) y, y)\right)=0 .
$$

Then $f \in C^{2}(\mathscr{U}), \frac{\partial f(x)}{\partial x}=g(x)$ and $\frac{\partial^{2} f(x)}{\partial x^{2}}=h(x)$.

Remark. We do not assume that (5.37) holds uniformly in $x$.

Proof. The result is well-known. We include a proof for the convenience of the reader. It suffices to consider a ball compactly contained in $\mathscr{U}$ and one may assume uniform continuity on that ball.

Step $1(p=1)$. In this case we may assume that $h \equiv 0$. Otherwise, choose $\tilde{f} \in C^{2}$ with $\tilde{f}^{\prime \prime}=h$ and consider $f-\tilde{f}$. Moreover, it follows from (5.37) that $f$ is differentiable with derivative $g$; thus $f \in C^{1}, f^{\prime}=g$. We assert that $f^{\prime}$ is both increasing and decreasing and thus constant. Assume $f^{\prime}$ is not increasing. Then there exist $a<b$, and $\delta>0$ such that $f^{\prime}(b)<f^{\prime}(a)+\delta(b-a)$. Let $c$ be the value where $f^{\prime}(x)+\delta x$ attains its maximum in $[a, b]$. Then $c<b$ and there is an $\varepsilon>0$ such that $f^{\prime}(c+t)+\delta t \leqq f^{\prime}(c), 0 \leqq t \leqq \varepsilon$. Set $R(c, t)=$ $f(c+t)-f(c)-f^{\prime}(c) t+\frac{1}{2} \delta t^{2}$. Then $R(c, 0)=\frac{\partial R}{\partial t}(c, 0)=0$ (since $f \in C^{1}$ ) 
and $\frac{\partial R}{\partial t}(c, t) \leqq 0$ if $0 \leqq t \leqq \varepsilon$, so that $R(c, t) \leqq 0$ for $0 \leqq t \leqq \varepsilon$. On the other hand, by (5.37),

$$
\lim _{t \rightarrow 0} t^{-2} R(c, t)=\frac{1}{2} \delta>0 .
$$

This is a contradiction, so $f^{\prime}$ must be increasing. The same argument applies to $-f^{\prime}$ and the assertion follows.

Step $2(p>1)$. Let $|y|=1$. By Step 1 the function $t \mapsto f(x+t y)$ is in $C^{2}$ and by the Taylor expansion,

$$
\begin{aligned}
t^{-2} \mid f(x+t y)-f & (x)-\operatorname{tg}(x) y-\frac{1}{2} t^{2}(h(x) y, y) \mid \\
& \leqq \int_{0}^{1}(1-s)|(h(x+s t g) y, y)-(h(x) y, y)| d s \\
& \leqq \frac{1}{2} \sup _{|z| \leqq t}|h(x+z)-h(x)| \leqq \omega(t),
\end{aligned}
$$

with $\omega(t) \rightarrow 0$ as $t \rightarrow 0$ by the uniform continuity of $h$. Let $\rho$ be a standard mollifier, i.e., let $\rho \in C_{0}^{\infty}\left(\mathbb{R}^{p}\right), \int_{\mathbb{R}^{p}} \rho(x) d x=1, \quad \rho^{\varepsilon}(x)=\varepsilon^{-p} \rho(x / \varepsilon)$. For $f^{\varepsilon}=\rho^{\varepsilon} * f$, we find

$$
t^{-2}\left|f^{\varepsilon}(x+t y)-f^{\varepsilon}(x)-\operatorname{tg}^{\varepsilon}(x) y-\frac{1}{2} t^{2}\left(h^{\varepsilon}(x) y, y\right)\right| \leqq \omega(t)
$$

as $t \rightarrow 0$, where $g^{\varepsilon}=\rho^{\varepsilon} * g, h^{\varepsilon}=\rho^{\varepsilon} * h$. Thus $D f^{\varepsilon}=g^{\varepsilon}, D^{2} f^{\varepsilon}=h^{\varepsilon}$. It follows that $f^{\varepsilon}$ is a Cauchy sequence in $C^{2}$ with limit $f$. Thus $f \in C^{2}$ with the obvious derivatives.

Proof of Proposition 5.5. Recall that if $f_{j} \rightarrow f$ boundedly a.e. and $g_{j} \rightarrow g$ in $L^{2}$, then $f_{j} g_{j} \rightarrow f g$ in $L^{2}$. It follows from the Euler-Lagrange equation that

$$
\int_{Y}\left(\mathbb{H}^{j}(x)\left(G+\nabla \psi^{j}\right), \nabla \eta\right) d x=0 \quad \forall \eta \in H_{\#}^{1} .
$$

In particular, $\left\|\nabla \psi^{j}\right\| \leqq C$, so that $\psi^{j}-\psi$ in $H^{1}$ for a subsequence. Since $\mathbb{L}^{j} \rightarrow \amalg$ boundedly a.e.,

$$
\int_{Y}(\mathbb{L}(x)(G+\nabla \psi), \nabla \eta) d x=0 \quad \forall \eta \in H_{\sharp}^{1},
$$

and thus $\psi$ minimizes the limit functional (the minimizer being unique by the lower bound on $\left\lfloor^{j}\right)$. Moreover,

$$
\begin{aligned}
& \int_{Y}\left(\mathbb{L}^{j}(x)\right.(G+\nabla \psi), \nabla \eta) d x \\
&=\int_{Y}\left(\left(\mathbb{L}^{j}(x)-\mathbb{L}(x)\right)(G+\nabla \psi), \nabla \eta\right) d x+\int_{Y}(\mathbb{L}(x)(G+\nabla \psi), \nabla \eta) d x .
\end{aligned}
$$

Observe that the last term on the right is zero, subtract (5.38) and choose $\eta=\psi^{j}-\psi$ to obtain

$$
\begin{aligned}
\left\|\psi-\psi_{j}\right\|_{H^{1}}^{2} & \leqq C \int_{Y}\left(\mathbb{U}^{j}(x)\left(\nabla\left(\psi-\psi^{j}\right)\right), \nabla\left(\psi-\psi^{j}\right)\right) d x \\
& =\int_{Y}\left(\left(\mathbb{L}^{j}(x)-\amalg_{-}(x)\right)(G+\nabla \psi), \nabla\left(\psi-\psi^{j}\right)\right) d x .
\end{aligned}
$$


Now $\left(\mathbb{L}^{j}-\mathbb{L}\right)(G+\nabla \psi) \rightarrow 0$ in $L^{2}, \nabla \psi^{j}-\nabla \psi \rightarrow 0$ in $L^{2}$, so that $\psi_{j} \rightarrow \psi$ in $H_{\# \text {. }}^{1}$. This holds along any weakly convergent subsequence. Thus $\psi^{j} \rightarrow \psi$ in $H_{\#}^{1}$ for the whole sequence.

\section{Application to a Layered Composite}

The present section complements the theoretical results given in Sections $3-5$. The example presented deals with a layered composite stretched by $\lambda$ in the direction of its layers, under plane strain conditions. In this case, one is able to perform all the required calculations for $\alpha_{\perp}^{s}$ (see (3.40)), $\Lambda_{5}^{s}$ (see (3.33)), $\Lambda_{6}^{s}$ (see (3.34)) and the homogenized moduli tensor $\mathbb{M}^{s}$ (see (3.35)) analytically and without any simplifying assumption.

Section 6 is divided into six subsections. In subsection 6.1 after a general description of the model we show some consequences of the ellipticity assumption of the incremental moduli tensor $\amalg(\lambda)=\partial^{2} W / \partial F^{2}$ and we compute the best stratified ellipticity constant $\alpha_{\perp(\lambda)}^{s}$ (see (6.5)). The incremental moduli of each layer are needed for the evaluation of the homogenized moduli of the composite and the corresponding formula for $M_{i j k l}(\lambda)$ are given in (6.6). Recall that the load parameter $\lambda$ in this problem is the stretch ratio in the lamination direction of the composite.

Corresponding to $\mathbb{L}(\lambda)$ we can define $\Lambda(\lambda)=\Lambda_{1}^{s}(\lambda)$ (see (3.31) and Theorem 3.7(i)). Its calculation is presented in Subsection 6.2 and is given in three steps. In Step 1, the minimum root $\hat{\Lambda}\left(\omega_{1}, \omega_{2}\right)$ of $(6.15)$ is calculated, where $\omega_{1}, \omega_{2}$ are the dimensionless wave numbers along $x_{1}, x_{2}$ of the eigenmode corresponding to the eigenvalue $\hat{\hat{A}}$. In Step 2, we seek $\hat{\Lambda}\left(\omega_{1}\right)$, which is found by the minimization of $\hat{\hat{\Lambda}}$ with respect to $\omega_{2} \in[0,2 \pi)$. The corresponding results are given by (6.26), (6.27). Finally in Step 3, the desired $\Lambda$ is found from $\hat{\Lambda}$ by a numerical search in a large enough interval of $\mathbb{R}$. Also in the same subsection $\Lambda_{6}^{s}(\lambda)$ (see (3.34)) is calculated, followed by an independent proof of the equality $\alpha_{\mathrm{L}(\lambda)}^{s}=\Lambda_{6}^{s}(\lambda)$ (see Theorem 3.7 (vii)).

Subsections 6.3 and 6.4 deal with the calculation of $\Lambda_{5}^{s}(\lambda)$ (see (3.33)) and $\alpha_{M}^{s}(\lambda)$ (see (3.36)). As it turns out from (6.25), $\lim _{\varepsilon \rightarrow 0} \hat{\hat{\Lambda}}\left(\lambda, \varepsilon \omega_{1}, \varepsilon \omega_{2}\right)=$ $\Lambda_{5}\left(\lambda, \omega_{1}, \omega_{2}\right)$ and $\Lambda_{5}^{s}(\lambda)$ can be found by minimizing $\hat{\Lambda}_{5}$ on $|\omega|=1$, $\omega_{1} \neq 0$, as shown in (6.34). The best stratified ellipticity parameter $\alpha_{M}^{s}(\lambda)$ of the homogenized composite defined by (6.41) is calculated with the help of (6.42).

In subsection 6.5 we calculate the critical stretch ratios $\lambda_{c}^{+}, \lambda_{c}^{-}$, which correspond to the onset of the first bifurcation of the composite in tension and compression (see definition (6.43) and results in (6.44)). We also calculate the critical stretch ratios $\lambda_{h}^{+}, \lambda_{h}^{-}$, which correspond to the first long-wavelength bifurcation of the composite in tension and compression (see definition (6.45) and results in (6.46)). In the same subsection we also define the critical stretch ratios $\lambda_{H}^{+}, \lambda_{\bar{H}}$ corresponding to the first loss of rank-1 convexity of the homogenized composite (see definition in (6.48) and results in (6.49)) and 
show that they concide with $\lambda_{h}^{+}, \lambda_{h}^{-}$, respectively (see (6.51)), thus providing for this application an independent proof of Theorem 3.7 (vi).

The last Subsection 6.6 uses a particular energy density function $W$ to calculate $\Lambda(\lambda), \lambda_{5}^{s}(\lambda), \lambda_{6}^{s}(\lambda)$ and $\alpha_{M}^{s}(\lambda)$ for stratified composites under tension and compression. For the particular choice of $W$ adopted here only the compression case gives interesting results, while for tension $\Lambda=\Lambda_{5}^{s}(\lambda)=$ $\Lambda_{6}^{s}(\lambda)=$ constant independent of $\lambda$.

\subsection{Model - General considerations, calculation of $M$}

Consider a composite medium made of a self-repeating sequence of elastic layers $\ell_{1}, \ell_{2}, \ldots, \ell_{n}$ with initial thicknesses $H, H, \ldots, H$ and current thicknesses $\ell_{1} \ell_{2} \quad \ell_{n} \quad \ell_{n}$ $h, h, \ldots, h$. The initial thickness of the base cell is $1=H+H+\cdots+H$ and its current thickness is $h=h+h+\cdots+h$. For simplicity only three layers $a, b, c$ per base cell are depicted in Fig. 6.1.

The composite deforms under plane strain conditions. Each layer is made of an isotropic, nonlinearly elastic material that remains strongly elliptic at any level of strain. There is perfect bonding between the layers, i.e., the vector traction and displacement are continuous across each interface for all possible deformations.

At the state of deformation that corresponds to the principal solution, which is depicted in Fig. 6.1, the $p^{\text {th }}$ layer is under principal stretches

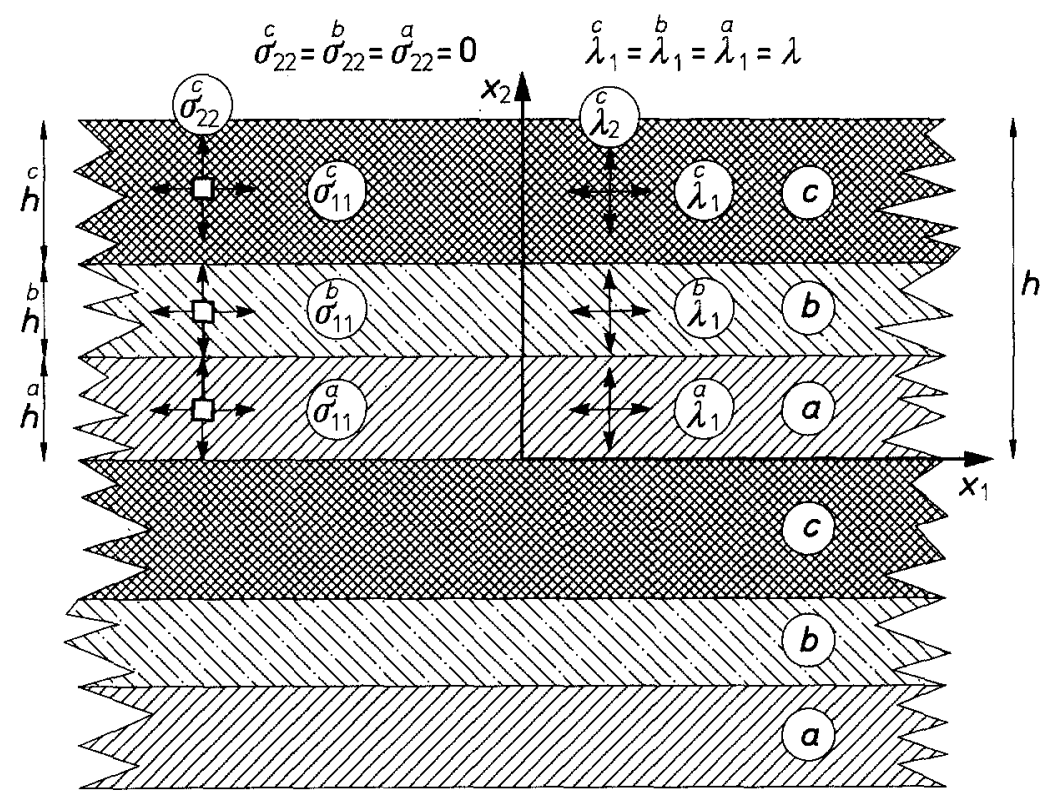

Fig. 6.1. Schematic drawing of the periodic layered composite. 
$\ell_{p} \ell_{p}$, $\lambda_{2}$ along the $x_{1}, x_{2}$ directions respectively. The assumed perfect bonding condition between layers implies that

$$
\stackrel{\ell_{1}}{\lambda_{1}}=\lambda_{1}^{\ell_{2}}=\cdots=\lambda_{1} \equiv \lambda \text {. }
$$

The corresponding principal Cauchy stresses in each layer are denoted by $\sigma_{11}^{\ell_{p}}, \sigma_{22}^{\ell_{p}}$. Traction continuity across the interfaces requires that

$$
\sigma_{22}^{\ell_{1}}=\sigma_{22}^{\ell_{2}}=\cdots=\sigma_{22}^{\ell_{n}} \equiv \sigma \text {. }
$$

A compression of the composite corresponds to $\lambda<1$, a tension corresponds to $\lambda>1$ while at $\lambda=1$ the composite is stress-free (when $\sigma=0$ ).

The principal stress and deformation state of the composite is completely determined by the initial geometry (i.e., the layer thicknesses $H, H, \ldots, H$ ), the material properties of each layer (i.e., the energy densities $\stackrel{\ell_{1}}{W}, W, \ldots, W$ ) and the load parameter (i.e., the $x_{1}$-stretch ratio $\lambda$ ). An isotropic nonlinearly hyperelastic material under plane strain conditions, has an energy density that is a function $W\left(l_{I}, l_{I I}\right)$ of the invariants of the Cauchy deformation tensor $F^{T} F$.

Noting that within each layer the deformation gradient tensor is $F=F(\lambda)=\operatorname{diag}\left[\lambda_{1}, \lambda_{2}\right]$, and so $l_{I}=\lambda_{1}^{2}+\lambda_{2}^{2}, l_{I I}=\lambda_{1}^{2} \lambda_{2}^{2}$, one finds (omitting the superscripts for notational simplicity) the nonzero components of the Cauchy stress tensor to be $\sigma_{11}$ and $\sigma_{22}$ and the nonzero components of the linearized elastic tensor at $F$ (also called the incremental moduli tensor) $\mathbb{L}=\mathbb{U}_{F(\lambda)}=\mathbb{L}(\lambda)=\partial^{2} W / \partial F^{2}$ to be

$$
L_{1111}, \quad L_{1122}=L_{2211}, \quad L_{2222}, \quad L_{1212}=L_{2121}, \quad L_{1221}=L_{2112} .
$$

Let us explicitly remember the following assumption:

(E) At every level of strain $F=F(\lambda)$ the linearized elastic tensor $\mathbb{H}_{F(\lambda)}(x)=$ $\partial^{2} W / \partial F^{2}$ is strongly elliptic in each layer.

From this assumption, one deduces (see (3.7)) that the $2 \times 2$ matrices $L_{i j k l} b_{j} b_{l}$ are positive-definite for all $\boldsymbol{b} \neq 0$. This implies that their principal minors are positive. In view of the arbitrariness of $\boldsymbol{b}$, one has

$$
\begin{gathered}
L_{1111}^{\ell_{p}}(\lambda), \quad L_{1212}^{\ell_{p}}(\lambda)=L_{2121}^{\ell_{p}}(\lambda), \quad L_{2222}^{\ell_{p}}(\lambda) \geqq \alpha_{11(\lambda)}>0, \\
\left.L_{1111}^{\ell_{p}}(\lambda) L_{1212}^{\ell_{p}}(\lambda)\right]\left(b_{1}\right)^{4}+\left[L_{1212}^{\ell_{p}}(\lambda) L_{2222}^{\ell_{p}}(\lambda)\right]\left(b_{2}\right)^{4} \\
+\left[L_{1111}^{\ell_{p}}(\lambda) L_{2222}^{\ell_{p}}(\lambda)+\left(L_{1212}^{\ell_{p}}(\lambda)\right)^{2}-\left(L_{1122}^{\ell_{p}}(\lambda)+L_{1221}^{\ell_{p}}(\lambda)\right)^{2}\right]\left(b_{1} b_{2}\right)^{2}>0 .
\end{gathered}
$$

Moreover, the implicit function theorem implies that near $\lambda=1, \sigma=0$ the stretch $\lambda_{2}$ is a function of $\lambda$. Therefore, of the two independent control parameters $\lambda$ and $\sigma$ for this problem, the stretch ratio $\lambda$ is chosen to be the load parameter. Also for reasons of simplicity, it is assumed that the lateral stress $\sigma=0$. 
From Theorem 3.7 (vii) it follows that the best stratified ellipticity constant for $\llbracket(\lambda)$ (see $(3.40)$ ) is

$$
\alpha_{\mathbb{L}(\lambda)}^{s}=\min \left\{L_{1212}^{\ell_{p}}(\lambda), L_{2222}^{\ell_{p}}(\lambda) \mid p=1,2, \ldots, n\right\} \geqq \alpha_{\mathbb{U}(\lambda)}>0 .
$$

Knowing the incremental moduli for each layer of the composite, one can find the corresponding homogenized incremental moduli $M_{F}=M(\lambda)=\mathbb{M}$. From (3.35) the nonzero components $M_{i j k l}$ of $M$ for the layered composite at hand, are found to be

$$
\begin{aligned}
& M_{1111}=\left\langle L_{1111}-\left(L_{1122}\right)^{2}\left(L_{2222}\right)^{-1}\right\rangle+\left\langle L_{1122}\left(L_{2222}\right)^{-1}\right\rangle^{2}\left\langle\left(L_{2222}\right)^{-1}\right\rangle^{-1}, \\
& M_{1122}=\left\langle L_{1122}\left(L_{2222}\right)^{-1}\right\rangle\left\langle\left(L_{2222}\right)^{-1}\right\rangle^{-1}=M_{2211}, \\
& M_{2222}=\left\langle\left(L_{2222}\right)^{-1}\right\rangle^{-1}, \\
& M_{2121}=\left\langle L_{2121}-\left(L_{1221}\right)^{2}\left(L_{1212}\right)^{-1}\right\rangle+\left\langle L_{1221}\left(L_{1212}\right)^{-1}\right\rangle^{2}\left\langle\left(L_{1212}\right)^{-1}\right\rangle^{-1}, \\
& M_{1221}=\left\langle L_{1221}\left(L_{1212}\right)^{-1}\right\rangle\left\langle\left(L_{1212}\right)^{-1}\right\rangle^{-1}=M_{2112}, \\
& M_{1212}=\left\langle\left(L_{1212}\right)^{-1}\right\rangle^{-1} .
\end{aligned}
$$

In this set of equations, $\langle f\rangle$ denotes the average of a function $f(x)$ defined in the interval $[0,1]$. For a function $f$ which is constant in each layer of the composite $\langle f\rangle=H f+H f^{2}+\cdots+H f^{\prime}$.

The derivation of (6.6) from (3.35) is straightforward and no details need to be given here.

\subsection{Calculation of $\Lambda_{1}^{s}, \Lambda_{6}^{s}$}

Attention is next focused on the calculation of $\Lambda_{1}^{s}(\lambda)$ corresponding to $\Perp(\lambda)$ (see (3.31)) for the layered composite under investigation. We always assume that $\Lambda_{1}^{s}(\lambda) \geqq 0$. From Theorem 3.7 (i) it then follows $\Lambda(\lambda)=\Lambda_{1}^{s}(\lambda)$, and so

$$
\begin{gathered}
\Lambda(\lambda)=\inf \left\{\hat{\Lambda}\left(\lambda, \omega_{1}\right) \mid \omega_{1} \in \mathbb{R}\right\}, \\
\hat{\Lambda}\left(\lambda, \omega_{1}\right)=\inf \left\{\hat{\Lambda}\left(\lambda, \omega_{1}, \omega_{2}\right) \mid \omega_{2} \in[0,2 \pi[\},\right. \\
\hat{\Lambda}\left(\lambda, \omega_{1}, \omega_{2}\right)=\inf \left\{Q(\nabla v ; Y) \mid v=e^{i\left(\omega_{1} \cdot x_{1}+\omega_{2} \cdot x_{2}\right)} p\left(x_{2}\right), p \in \mathscr{H}_{\#}^{1}\right\} .
\end{gathered}
$$

Two cases can occur: Either $\Lambda=\Lambda_{6}^{s}$ where $\Lambda_{6}^{s}$ is defined by (3.34) or $\Lambda<\Lambda_{6}^{s}$. In the former case $\Lambda=\alpha_{\mathrm{L}}^{s}$ is given by Theorem 3.7 (vii) (cf. (6.5)). In the latter case a formula for $\hat{\Lambda}\left(\omega_{1}\right)$ will be derived and $\Lambda$ is obtained by minimization over $\omega_{1}$. In the specific examples discussed in Section 6.5 it was found that $\Lambda=\Lambda_{6}^{s}$ in extension while $A<\Lambda_{6}^{s}$ in compression. 
Assuming now that $\Lambda<\Lambda_{6}^{s}(\lambda)$ and restricting attention to values of $\left(\omega_{1}, \omega_{2}\right)$ for which $0 \leqq \hat{\Lambda}\left(\lambda, \omega_{1}, \omega_{2}\right)<\Lambda_{6}^{s}(\lambda)$ one easily checks that the infimum in (6.9) is attained (cf. the proofs of Theorem 3.4(ii), (iii), Sections 4.5 and 4.6) and that $\hat{\Lambda}$ depends continuously on $\omega_{2}$, so that the infimum in the definition of $\hat{\Lambda}$ is attained, i.e.,

$$
\hat{\Lambda}\left(\lambda, \omega_{1}\right)=\hat{\Lambda}\left(\lambda, \omega_{1}, \omega_{2, m}\right),
$$

where $\omega_{2, m}$ depends on $\omega_{1}$ and $\lambda$.

The Euler-Lagrange equations for the minimizer $\hat{\boldsymbol{v}}\left(x_{2}\right)=e^{i \omega_{2} \cdot x_{2}} \hat{\boldsymbol{p}}\left(x_{2}\right)$ in (6.9) are

$$
\begin{aligned}
& -\omega_{1}^{2}\left(L_{1111}-\hat{\hat{\Lambda}}\right) \hat{v}_{1}+i \omega_{1}\left(L_{1122}+L_{1221}\right) \hat{v}_{2,2}+\left(L_{1212}-\hat{\Lambda}\right) \hat{v}_{1,22}=0, \\
& -\omega_{1}^{2}\left(L_{2121}-\hat{\Lambda}\right) \hat{v}_{2}+i \omega_{1}\left(L_{2112}+L_{2211}\right) \hat{v}_{1,2}+\left(L_{2222}-\hat{\Lambda}\right) \hat{v}_{2,22}=0,
\end{aligned}
$$

for $\left.x_{2} \in\right] 0, \stackrel{\ell_{1}}{H}[\cup] \stackrel{\ell_{1}}{H}, \stackrel{\ell_{1}}{H}+\stackrel{\ell_{2}}{H}\left[\cup \cdots \cup \stackrel{\ell_{1}}{H}+\stackrel{\ell_{2}}{H}+\cdots+\stackrel{\ell_{n-1}}{H}, 1\right.$ [. The corresponding interface conditions are

$$
\begin{array}{ll}
\llbracket\left(L_{1212}-\hat{\hat{\Lambda}}\right) \hat{v}_{1,2}+i \omega_{1} L_{1221} \hat{v} \rrbracket=0, & \llbracket \hat{v}_{1} \rrbracket=0, \\
\llbracket i \omega_{1} L_{2211} \hat{v}+\left(L_{2222}-\hat{\hat{A}}\right) \hat{v}_{2,2} \rrbracket=0, & \llbracket \hat{v}_{2} \rrbracket=0,
\end{array}
$$

for $x_{2}=\stackrel{\ell_{1}}{H}, \stackrel{\ell_{1}}{H}+\stackrel{\ell_{2}}{H}, \ldots, \stackrel{\ell_{1}}{H}+\stackrel{\ell_{2}}{H}+\cdots+\stackrel{\ell_{n-1}}{H}$. The boundary conditions at the ends $x_{2}=0$ and $x_{2}=1$ of the unit cell, as a direct consequence of the particular form of $\hat{v}\left(x_{2}\right)$, are

$$
\left[\left(L_{1212}-\hat{\hat{\Lambda}}\right) \hat{v}_{1,2}+i \omega_{1} L_{1221} \hat{v}_{2}\right](1)=\exp \left(i \omega_{2}\right)\left[\left(L_{1212}-\hat{\hat{\Lambda}}\right) \hat{v}_{1,2}+i \omega_{1} L_{1221} \hat{v}_{2}\right](0),
$$

$\left[i \omega_{1} L_{2211} \hat{v}_{1}+\left(L_{2222}-\hat{\hat{\Lambda}}\right) \hat{v}_{2,2}\right](1)=\exp \left(i \omega_{2}\right)\left[i \omega_{1} L_{2211} \hat{v}_{2}+\left(L_{2222}-\hat{\Lambda}\right) \hat{v}_{2,2}\right](0)$,

$$
\hat{\boldsymbol{v}}(1)=\exp \left(i \omega_{2}\right) \hat{\boldsymbol{v}}(0) \text {. }
$$

In each layer $\ell_{p}, p=1, \ldots, n$, we consider the biquadratic equation associated with (6.10):

$$
\begin{aligned}
& \left(L_{1212}-\hat{\hat{\Lambda}}\right)\left(L_{2222}-\hat{\hat{\Lambda}}\right) z^{4} \\
& +\left[\left(L_{1111}-\hat{\Lambda}\right)\left(L_{2222}-\hat{\hat{\Lambda}}\right)+\left(L_{1212}-\hat{\Lambda}\right)\left(L_{2121}-\hat{\hat{\Lambda}}\right)-\left(L_{1122}+L_{1221}\right)^{2}\right] z^{2} \\
& +\left(L_{1111}-\hat{\hat{\Lambda}}\right)\left(L_{2121}-\hat{\Lambda}\right)=0
\end{aligned}
$$

whose roots $z_{1}, z_{2}, z_{3}=-z_{1}, z_{4}=-z_{2}$ are generally complex, at least for relatively small values of $\hat{\hat{\Lambda}}$, as follows from (E) (see (6.4)). We shall also need the functions defined for $z_{1} \neq z_{2} \neq 0$ by

$$
\begin{aligned}
& a_{1}\left(\omega ; z_{1}, z_{2}\right)=\frac{z_{1}^{2} \cos \left(\omega z_{2}\right)-z_{2}^{2} \cos \left(\omega z_{1}\right)}{z_{1}^{2}-z_{2}^{2}}, \\
& a_{2}\left(w ; z_{1}, z_{2}\right)=\frac{\cos \left(\omega z_{1}\right)-\cos \left(\omega z_{2}\right)}{z_{1}^{2}-z_{2}^{2}},
\end{aligned}
$$




$$
\begin{aligned}
& b_{1}\left(\omega ; z_{1}, z_{2}\right)=\frac{z_{2} \sin \left(\omega z_{1}\right)-z_{1} \sin \left(\omega z_{2}\right)}{z_{1}^{2}-z_{2}^{2}}, \\
& b_{2}\left(\omega ; z_{1}, z_{2}\right)=\frac{z_{1} \sin \left(\omega z_{1}\right)-z_{2} \sin \left(\omega z_{2}\right)}{z_{1}^{2}-z_{2}^{2}}
\end{aligned}
$$

and whose definition has to be extended by continuity for $z_{1}=0$ and $z_{1}=z_{2}$.

Finally we define in each layer the $4 \times 4$ matrix $\ell_{p} \mathbf{K}=\mathbf{K}\left(\lambda ; \omega_{1}, y\right)$ whose entries are

$$
\begin{aligned}
K_{11}= & K_{33}=a_{1}\left(\omega_{1} ; z_{1}, z_{2}\right) \\
& +a_{2}\left(\omega_{1} ; z_{1}, z_{2}\right) \frac{L_{1111}\left(L_{1122}+L_{1221}\right)-\left(L_{2222}-y\right)\left(L_{1111}-y\right)}{\left(L_{2222}-y\right)\left(L_{1212}-y\right)}, \\
K_{12}= & -K_{43}=i b_{1}\left(\omega_{1} ; z_{1}, z_{2}\right) \frac{L_{1122}}{\left(L_{2222}-y\right)}-i b_{2}\left(\omega_{1} ; z_{1}, z_{2}\right) \frac{L_{1221}}{\left(L_{1212}-y\right)}, \\
K_{13}= & i \frac{b_{1}\left(\omega_{1} ; z_{1}, z_{2}\right)}{z_{1} z_{2}} \frac{1}{\left(L_{2222}-y\right)}+i b_{2}\left(\omega_{1} ; z_{1}, z_{2}\right) \frac{1}{\left(L_{1212}-y\right)}, \\
K_{14}= & -K_{23}=-a_{2}\left(\omega_{1} ; z_{1}, z_{2}\right) \frac{\left(L_{1122}+L_{1221}\right)}{\left(L_{1212}-y\right)\left(L_{2222}-y\right)}, \\
K_{21}= & -K_{34}=i b_{1}\left(\omega_{1} ; z_{1}, z_{2}\right) z_{1} z_{2} \frac{L_{1221}}{\left(L_{1212}-y\right)}-i b_{2}\left(\omega_{1} ; z_{1}, z_{2}\right) \frac{L_{1122}}{\left(L_{2222}-y\right)}, \\
K_{22}= & K_{44}=a_{1}\left(\omega_{1} ; z_{1}, z_{2}\right) \\
& -a_{2}\left(\omega_{1} ; z_{1}, z_{2}\right) \frac{L_{1122}\left(L_{1122}+L_{1221}\right)-\left(L_{2222}-y\right)\left(L_{1111}-y\right)}{\left(L_{2222}-y\right)\left(L_{1212}-y\right)}, \\
K_{24}= & -i b_{1}\left(\omega_{1} ; z_{1}, z_{2}\right) \frac{1}{\left(L_{1212}-y\right)}-i b_{2}\left(\omega_{1} ; z_{1}, z_{2}\right) \frac{1}{\left(L_{2222}-y\right)}, \\
K_{31}= & i b_{1}\left(\omega_{1} ; z_{1}, z_{2}\right) \frac{L_{1221}^{2}-\left(L_{1212}-y\right)^{2}}{\left(L_{1212}-y\right)} \\
& +i b_{2}\left(\omega_{1} ; z_{1}, z_{2}\right) \frac{L_{1122}^{2}-\left(L_{1111}-y\right)\left(L_{2222}-y\right)}{\left(L_{2222}-y\right)}, \\
K_{42}= & \left.-i b_{1}\left(\omega_{1} ; z_{1}, z_{2}\right) \frac{\left.L_{1122}^{2}-\left(\omega_{1} ; z_{1}, z_{2}\right) \frac{L_{1221}^{2}-\left(L_{1212}-y\right)^{2}}{\left(L_{12222}-y\right)}, y\right)}{\left(L_{1212}-y\right)\left(L_{2222}-y\right)}, y\right) \\
& \times \frac{\left(L_{1212}-y\right)^{2} L_{1122}+\left(L_{111}-y\right)\left(L_{2222}-y\right) L_{1221}-L_{1221} L_{1122}\left(L_{1221}+L_{1122}\right)}{K_{32}=} \\
& K_{41}=-a_{2}\left(\omega_{1} ; z_{1}, z_{2}\right)
\end{aligned}
$$


Note that as in previous formulae superscripts are in general omitted for the sake of notational simplicity.

Proposition 6.1. (i) Under the previously stated notations and assumptions, $\hat{\Lambda}$ is the smallest nonnegative root of

$$
\operatorname{det}\left[\mathbf{F}\left(\lambda, \omega_{1}, \hat{\Lambda}\right)-\exp \left(i \omega_{2}\right) \mathbf{l}\right]=0,
$$

where $\mid$ denotes the unit $4 \times 4$ matrix and $\mathbf{F}=\mathbf{F}\left(\lambda, \omega_{1}, \hat{\hat{\Lambda}}\right)$ is defined by

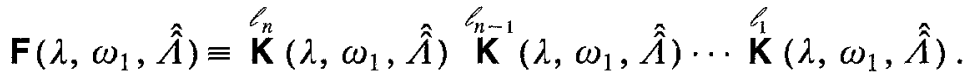

(ii)

$$
\operatorname{det} \stackrel{\ell_{p}}{\mathbf{K}}=1, \quad\left(\stackrel{\ell_{p}}{\mathbf{K}}\right)^{-1}\left(\lambda ; \omega_{1}, \hat{\hat{\Lambda}}\right)=\stackrel{\ell_{p}}{\mathbf{K}}\left(\lambda,-\omega_{1}, \hat{\hat{\Lambda}}\right) .
$$

(iii) All the invariants of $\mathbf{F}$ are always real and satisfy

$$
\begin{gathered}
I_{1}^{\mathrm{F}}=I_{3}^{\mathrm{F}}=\operatorname{tr} \mathbf{F}, \quad I_{4}^{\mathrm{F}}=1, \\
I_{1}^{\mathrm{F}}\left(\lambda ; \omega_{1}, \hat{\Lambda}\right)=I_{1}^{\mathrm{F}}\left(\lambda ;-\omega_{1}, \hat{\Lambda}\right), \quad I_{2}^{\mathrm{F}}\left(\lambda ; \omega_{1}, \hat{\Lambda}\right)=I_{2}^{\mathrm{F}}\left(\lambda ;-\omega_{1}, \hat{\Lambda}\right) .
\end{gathered}
$$

Proof. We assume for simplicity that in all the layers all the roots of the biquadratic equation (6.13) are simple. In the case of multiple roots one just has to verify that the final formulae obtained by taking the limits $z_{1} \rightarrow z_{2}$ and $z_{1} \rightarrow 0$ make sense.

(i) Under the previous assumptions the general solution of the system (6.10) of ordinary differential equations with coefficients constant in each layer $\ell_{p}$ is

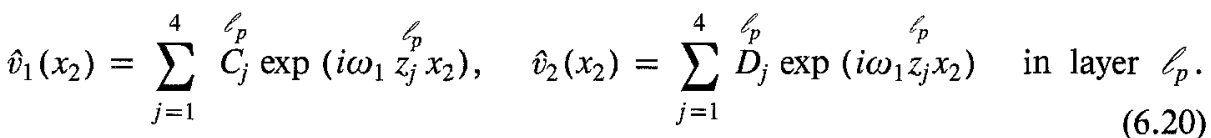

In $(6.20)$ the ${ }_{\ell_{p}}^{\ell_{j}}(j=1, \ldots, 4)$ are the four roots of $(6.13)$; the constants $C_{j}$ and $D_{j}(j=1, \ldots, 4)$ are related by

$$
\begin{aligned}
& C_{j}=-\frac{1}{\ell_{p}} \frac{\left(L_{2121}^{\ell_{p}}-\hat{\hat{\Lambda}}\right)+\left(z_{j}^{Z_{p}}\right)^{2}\left(L_{2222}^{\ell_{p}}-\hat{\hat{\Lambda}}\right)}{z_{j}}{\stackrel{\ell}{D_{j}}}_{j}^{\ell_{p}}, \\
& \frac{\ell_{p}}{D_{j}}=-\frac{1}{\ell_{p}} \frac{\left(L_{1111}^{\ell_{p}}-\hat{\hat{\Lambda}}\right)+\left(z_{j}^{\ell_{j}}\right)^{2}\left(L_{1212}^{\ell_{p}}-\hat{\hat{\Lambda}}\right)}{z_{j}^{\ell_{p}}}{\stackrel{C_{j}}{C_{j}}}^{\ell_{p}} .
\end{aligned}
$$

Substituting (6.20), (6.21) into the interface conditions (6.11) one obtains, in matrix form, the following equations for the interfaces $x_{2}=$ 
$\stackrel{\ell_{1}}{H}, \ldots, \stackrel{\ell_{1}}{H}+\stackrel{\ell_{2}}{H}+\cdots+\stackrel{\ell_{n-1}}{H}$, respectively:

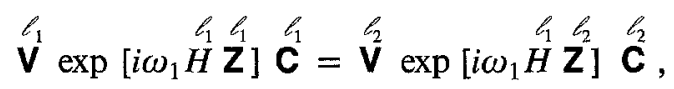

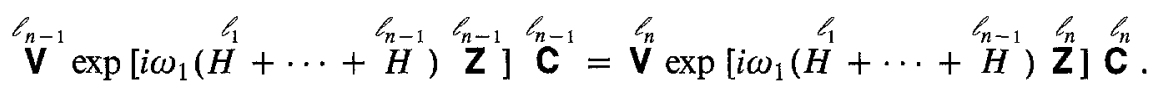

The superscripts $\ell_{p}$ in the $4 \times 4$ matrices $\mathbf{V}$ and $\mathbf{Z}$ appearing in (6.22) indicate the layer at which the components of these matrices have to be evaluated. These matrices have components

$$
\begin{aligned}
& V_{1 j}=1, \\
& V_{2 j}=\frac{\left(L_{1111}-\hat{\hat{\Lambda}}\right)+z_{j}^{2}\left(L_{1212}-\hat{\hat{\Lambda}}\right)}{z_{j}\left(L_{1122}+L_{1221}\right)}, \\
& V_{3 j}=z_{j}\left(L_{1212}-\hat{\hat{\Lambda}}\right)-L_{1221} \frac{\left(L_{1111}-\hat{\hat{\Lambda}}\right)+z_{j}^{2}\left(L_{1212}-\hat{\hat{\Lambda}}\right)}{z_{j}\left(L_{1122}+L_{1221}\right)}, \\
& V_{4 j}=L_{1122}-\left(L_{2222}-\hat{\hat{\Lambda}}\right) \frac{\left(L_{1111}-\hat{\hat{\Lambda}}\right)+z_{j}^{2}\left(L_{1212}-\hat{\hat{\Lambda}}\right)}{L_{1122}+L_{2222}}, \\
& Z_{i j}= \begin{cases}z_{i} & \text { for } i=j, \\
0 & \text { for } i \neq j .\end{cases}
\end{aligned}
$$

The 4-dimensional vectors $\stackrel{\ell_{p}}{\mathbf{C}}$ in (6.22) have as components the constants ${ }_{p}^{\ell_{p}}$ introduced in (6.21).

The end cell boundary condition (6.12) in conjunction with (6.20) furnishes the additional relation

$$
\mathbf{V}_{n} \exp \left[i \omega_{1} \mathbf{Z}_{n}\right] \mathbf{C}^{\ell_{n}}=\exp \left(i \omega_{2}\right) \stackrel{\ell_{1}}{\mathbf{V}} \mathbf{C}
$$

Laborious, although straightforward, algebra gives

$$
\mathbf{K}=\ell_{p}^{\ell_{p}} \exp \left[i \omega_{1} H \mathbf{Z}\right]\left(\mathbf{V}_{p}\right)^{-1}, \quad p=1,2, \ldots, n ;
$$

hence from (6.22) and (6.23) it follows that a nontrivial solution $\hat{v}\left(x_{2}\right)$ exists for (6.10), (6.11), (6.12) if and only if (6.15) is satisfied.

(ii) From (6.24) it follows immediately that (6.17) holds.

(iii) The only nontrivial property in (6.18) and (6.19) is $I_{3}^{F}=\operatorname{tr} F$. Since a simple inspection of the definition of $\mathbf{F}=\mathbf{K}_{n} \mathbf{K}^{\ell_{n-1}} \ldots \mathbf{K}_{1}$ implies that $\operatorname{tr} \mathbf{F}^{-1}=$ $\operatorname{tr}\left(\mathbf{K}_{1} \ldots \ell^{\ell_{n-1} \ell_{n}} \mathbf{K}\right)$, it is enough to prove

$$
\operatorname{tr}\left(\mathbf{K}_{1}^{\ell_{2}} \ldots \mathscr{K}_{n}\right)=\operatorname{tr}\left(\mathbf{K}_{n} \ldots \mathbf{K}^{\ell_{2}} \mathbf{K}_{1}\right)
$$


This follows from mathematical induction on the number of layers. To this end introduce the matrix

$$
\mathbf{A}^{*}=\left[\begin{array}{rrrr}
A_{33} & -A_{43} & A_{13} & -A_{23} \\
-A_{34} & A_{44} & -A_{14} & A_{24} \\
A_{31} & -A_{41} & A_{11} & -A_{21} \\
-A_{32} & A_{42} & -A_{12} & A_{22}
\end{array}\right]
$$

associated with any $4 \times 4$ matrix $\mathbf{A}$ with components $A_{i j}$. From the definition of $\mathbf{K}$ it follows that $\mathbf{K}=\mathbf{K}^{*}$. Assume that for $p$ layers, $\mathbf{K} \mathbf{K} \ldots \mathbf{K}=$ $(\mathbf{K} \ldots \mathbf{K} \mathbf{K})^{*}$. Then one can easily show that $(\mathbf{K} \mathbf{K} \ldots \mathbf{K}) \stackrel{\ell^{\prime}}{\mathbf{K}}=$ $\ell_{p+1} \ell_{p} \ell_{2} \ell_{1}$

[ $\mathbf{K}(\mathbf{K} \ldots \mathbf{K} \mathbf{K})]^{*}$. The desired property follows from the (obvious) remark that $\operatorname{tr}(\mathbf{A})=\operatorname{tr}\left(\mathbf{A}^{*}\right)$.

The use of (6.18) in (6.15) yields the following equation, implicit in $\hat{\hat{A}}$ :

$$
\begin{gathered}
\exp \left(4 i \omega_{2}\right)-I_{1}^{\mathrm{F}}\left(\lambda, \omega_{1}, \hat{\hat{\Lambda}}\right) \exp \left(3 i \omega_{2}\right)+I_{2}^{\mathrm{F}}\left(\lambda, \omega_{1}, \hat{\hat{\Lambda}}\right) \exp \left(2 i \omega_{2}\right) \\
-I_{1}^{\mathrm{F}}\left(\lambda, \omega_{1}, \hat{\hat{\Lambda}}\right) \exp \left(i \omega_{2}\right)+1=0 .
\end{gathered}
$$

This equation provides $\hat{\hat{\Lambda}}$ (defined in (6.9)) as a function of the load parameter $\lambda$ and the dimensionless wave numbers $\omega_{1}, \omega_{2}$ of the corresponding mode $\hat{v}\left(x_{2}\right)$. Once $\hat{\hat{A}}\left(\lambda, \omega_{1}, \omega_{2}\right)$ has been found, $\hat{\Lambda}\left(\lambda, \omega_{1}\right)$, its infimum over all $\omega_{2} \in[0,2 \pi)$, can be calculated. This calculation works as follows. In view of the discussion after (6.10) the infimum in the definition (6.8) of $\Lambda\left(\lambda, \omega_{1}\right)$ is achieved for some value $\omega_{2 m}$, in which case $\hat{\Lambda}\left(\lambda, \omega_{1}\right)=\hat{\Lambda}\left(\lambda, \omega_{1}, \omega_{2 m}\right)$. In this case $y_{m} \equiv \exp \left[i \omega_{2 m}\right]$ is an eigenvalue of $\mathbf{F}$ in (6.15), and hence it is a root of its characteristic polynomial $f(y) \equiv y^{4}-I_{1}^{\mathrm{F}} y^{3}+I_{2}^{\mathrm{F}} y^{2}-I_{1}^{\mathrm{F}} y+1$, i.e., $f\left(y_{m}\right)=0$. Two cases can occur:

a) $y_{m} \in \mathbb{R}$, which in view of the fact that $\left|y_{m}\right|=1$ implies that $y_{m}=+1$ or -1 and hence

$$
\begin{array}{ll}
2 I_{1}^{\mathrm{F}}\left(\lambda, \omega_{1}, \hat{\Lambda}\right)-I_{2}^{\mathrm{F}}\left(\lambda, \omega_{1}, \hat{\Lambda}\right)-2=0, & \omega_{2 m}=0, \\
2 I_{1}^{\mathrm{F}}\left(\lambda, \omega_{1}, \hat{\Lambda}\right)+I_{2}^{\mathrm{F}}\left(\lambda, \omega_{1}, \hat{\Lambda}\right)+2=0, & \omega_{2 m}=\pi .
\end{array}
$$

b) $y_{m} \in \mathbb{C} \backslash \mathbb{R}$, which in view of the fact that $\left|y_{m}\right|=1$ and the reality of $I_{1}^{\mathrm{F}}, I_{2}^{\mathrm{F}}$ implies that the four roots of the biquadratic polynomial $f(y)$ are $y_{m}$, $\bar{y}_{m},\left(y_{m}\right)^{-1},\left(\bar{y}_{m}\right)^{-1}$ with $\bar{y}_{m}=\left(y_{m}\right)^{-1}$. Using these relations in the expressions of the invariants as functions of the roots of $f(y)$ we obtain the following implicit equation for $\hat{A}$ :

$$
\frac{1}{4}\left[I_{1}^{\mathrm{F}}\left(\lambda, \omega_{1}, \hat{\Lambda}\right)\right]^{2}-I_{2}^{\mathrm{F}}\left(\lambda, \omega_{1}, \hat{\Lambda}\right)+2=0, \quad \omega_{2 m}= \pm \cos ^{-1}\left(\frac{I_{1}^{\mathrm{F}}}{4}\right) .
$$

Of course, for a root $\hat{\Lambda}$ of (6.27) to be acceptable, one should also verify that $\left|I_{1}^{\mathrm{F}}\left(\lambda, \omega_{1}, \hat{\Lambda}\right)\right| \leqq 4$. Consequently, the quantity $\hat{\Lambda}\left(\lambda, \omega_{1}\right)$ which is defined by (6.8) is the minimum nonnegative root of (6.26), (6.27). 
For the case where $\hat{\Lambda}$ satisfies (6.26), a physical interpretation can be given to the corresponding mode $v\left(x_{1}, x_{2}\right)$. If $(6.26)_{1}$ is satisfied at $\hat{\Lambda}$, then $\omega_{2 m}=0$ and from (6.9) it follows that $v\left(x_{1}, 0\right)=v\left(x_{1}, 1\right)$, which indicates a symmetric mode. If $(6.26)_{2}$ is satisfied at $\hat{\Lambda}$, then $\omega_{2 m}=\pi$ and from (6.9) it follows that $v\left(x_{1}, 0\right)=-v\left(x_{1}, 1\right)$, which indicates an antisymmetric mode. In all the numerical calculations performed for this example, $\hat{\Lambda}$ is always found to correspond to a symmetric or an antisymmetric mode $v$, as will be seen subsequently.

The calculation of $\Lambda(\lambda)$, which is defined in (6.7) as the infimum over all $\omega_{1}$ of $\hat{A}\left(\lambda, \omega_{1}\right)$, is done by a simple numerical search over an adequately large interval for $\omega_{1}$. From (6.19) it follows that only positive values of $\omega_{1}$ need to be considered in the abovementioned numerical search.

Remark. For $\omega_{1}=0, \hat{\hat{A}}$ can still be found from the original governing equations (6.10)-(6.12). In this case the governing equations for $\hat{v}_{1}\left(x_{2}\right)$ and $\hat{v}_{2}\left(x_{2}\right)$ decouple and $\hat{v}_{1}, \hat{v}_{2}$ are piecewise linear within each layer, as is easily seen from (6.10). The interface and boundary conditions (6.11), (6.12) finally imply that

$$
\hat{\hat{\Lambda}}\left(\lambda, 0, \omega_{2}\right)=\min \left\{L_{1212}^{\ell_{p}}(\lambda), L_{2222}^{\ell_{p}}(\lambda) \mid p=1,2, \ldots, n\right\}=\alpha_{\mathbb{L}(\lambda)}^{s} .
$$

Since $\hat{\Lambda}\left(\lambda, 0, \omega_{2}\right)=\hat{\hat{\Lambda}}(\lambda, 0,0)$ (in view of $(6.28)$ ), one can observe that $\hat{\Lambda}(\lambda, 0,0)=\Lambda_{6}^{s}$ where $\Lambda_{6}^{s}$ is defined in (3.34), and so we have an independent proof of Theorem 3.7(vii). It should also be noted that $\omega_{1}=0$ is a singular point in $\hat{\Lambda}\left(\lambda, \omega_{1}\right)$ since for the applications considered subsequently the $\lim _{\omega_{1} \rightarrow 0} \hat{\Lambda}\left(\lambda, \omega_{1}\right)$ exists and is different from (6.28). A physical interpretation of the existence of this singular point is not difficult if one observes that at the neighborhood of $\omega_{1}=0$ two physically different types of modes can be found from (6.9): the modes which are independent of $x_{1}$ as well as the long-wavelength type modes, i.e., modes whose wavelength in the $x_{1}$ direction is much larger that the unit cell size.

Hence in the numerical calculation of $\Lambda(\lambda)$ from $\hat{\Lambda}\left(\lambda, \omega_{1}\right)$, one has to keep in mind that $\hat{\Lambda}(\lambda, 0)$ is still given by the right-hand side of $(6.28)$ while for $\omega_{1}>0, \hat{\Lambda}\left(\lambda, \omega_{1}\right)$ is the minimum nonnegative root of (6.26), (6.27).

\subsection{Calculation of $\Lambda_{5}^{s}(\lambda)$}

For a fixed $\lambda$, we now study the determination of $\Lambda_{5}^{s}$ (see (3.32)), given in our case by

$$
\Lambda_{5}^{s}(\lambda) \equiv \liminf _{\left(\omega_{1}, \omega_{2}\right) \rightarrow 0} \hat{\Lambda}\left(\lambda, \omega_{1}, \omega_{2}\right) .
$$

The calculation is obviously nontrivial only when $\Lambda_{5}^{s}(\lambda)<\Lambda_{6}^{s}(\lambda)=\alpha_{\mathbb{U}(\lambda)}^{s}$ and therefore in the following we fix $\eta>0$ and we always assume that

$$
0 \leqq \hat{\Lambda}\left(\lambda, \omega_{1}, \omega_{2}\right) \leqq \Lambda_{6}^{s}(\lambda)-\eta ;
$$

from the remark after (6.28) it then follows that $\omega_{1} \neq 0$. 
We first define

$$
\hat{\Lambda}_{5}\left(\lambda, \omega_{1}, \omega_{2}\right) \equiv \liminf _{\varepsilon \rightarrow 0^{+}} \hat{\hat{\Lambda}}\left(\lambda, \varepsilon \omega_{1}, \varepsilon \omega_{2}\right) .
$$

We shall see in a moment that $\Lambda_{5}^{s}=\inf \left\{\hat{\Lambda}_{5}\left(\omega_{1}, \omega_{2}\right)|| \omega \mid=1, \omega_{1} \neq 0\right\}$ (see (6.34)). Note that this is not completely obvious, because in (6.30) we only consider limits along lines passing through the origin, while in (6.29) any sequence $\omega \rightarrow 0$ is taken into account.

We introduce the $4 \times 4$ matrix $\mathbf{G}=\mathbf{G}(\lambda, y)$ whose nonzero components are given by

$$
\begin{array}{ll}
G_{12}=\left\langle L_{1221}\left(L_{1212}-y\right)^{-1}\right\rangle, & G_{21}=\left\langle L_{1122}\left(L_{2222}-y\right)^{-1}\right\rangle, \\
G_{13}=\left\langle\left(L_{1212}-y\right)^{-1}\right\rangle, & G_{31}=-\left\langle\left(L_{1111}-y\right)-\left(L_{1122}\right)^{2}\left(L_{2222}-y\right)^{-1}\right\rangle, \\
G_{24}=-\left\langle\left(L_{2222}-y\right)^{-1}\right\rangle, & G_{42}=-\left\langle\left(L_{2121}-y\right)-\left(L_{1221}\right)^{2}\left(L_{1212}-y\right)^{-1}\right\rangle, \\
G_{34}=-\left\langle L_{1122}\left(L_{2222}-y\right)^{-1}\right\rangle, & G_{43}=-\left\langle L_{1221}\left(L_{1212}-y\right)^{-1}\right\rangle .
\end{array}
$$

Note that $\mathbf{G}$ is uniformly bounded for $0 \leqq y \leqq \Lambda_{6}^{s}(\lambda)-\eta$.

Proposition 6.2. (i) Under the previously stated notations and assumptions $\hat{\Lambda}_{5} \equiv$ $\hat{\Lambda}_{5}\left(\lambda, \omega_{1}, \omega_{2}\right)$ satisfies the implicit equation

$$
\operatorname{det}\left[\mathbf{G}\left(\lambda, \hat{\Lambda}_{5}\right)-\frac{\omega_{2}}{\omega_{1}} \mathbf{I}\right]=0
$$

(ii) The invariants of $\mathbf{G}$ are

$I_{1}^{\mathbf{G}}=0, \quad I_{2}^{\mathbf{G}}=-\frac{1}{2} \operatorname{tr} \mathbf{G}^{2}=-\left(G_{12} G_{21}+G_{13} G_{31}+G_{24} G_{42}+G_{34} G_{43}\right)$,

$I_{3}^{\mathbf{G}}=0, \quad I_{4}^{\mathbf{G}}=\frac{1}{8}\left[\left(\operatorname{tr} \mathbf{G}^{2}\right)^{2}-2 \operatorname{tr} \mathbf{G}^{4}\right]=\left(G_{12} G_{43}-G_{13} G_{42}\right)\left(G_{21} G_{34}-G_{31} G_{24}\right)$. (iii)

$$
\Lambda_{5}^{s}(\lambda)=\inf _{\substack{|\omega|=1 \\ \omega_{1} \neq 0}} \hat{\Lambda}_{5}\left(\lambda, \omega_{1}, \omega_{2}\right) .
$$

Proof. (i) From the definition of $\mathbf{F}\left(\lambda, \omega_{1}, \hat{\hat{\Lambda}}\right.$ ) (see (6.16)) it follows that $\left(y, \omega_{1}\right) \mapsto \mathbf{F}\left(\lambda, \omega_{1}, y\right)$ is a smooth function on $\mathscr{U} \times \mathbb{R}$ where $\mathscr{U}=$ $\left(0, \Lambda_{6}^{s}(\lambda)-\eta\right)$ and

$$
\left|\mathbf{F}\left(\lambda, \omega_{1}, y\right)-\left(\mathbf{I}+i \omega_{1} \mathbf{G}(\lambda, y)\right)\right| \leqq C\left|\omega_{1}\right|^{2} \quad \text { for all } y \in \mathscr{U} \text {. }
$$

Moreover,

$$
\left|\left(\mathbf{F}\left(\lambda, \omega_{1}, y\right)-e^{i \omega_{2}} \mid\right)-i\left(\omega_{1} \mathbf{G}(\lambda, y)-\omega_{2} \mathbf{I}\right)\right| \leqq C|\omega|^{2} .
$$

Since $\omega_{1} \neq 0$, formula (6.32) follows immediately.

(ii) The results are only a matter of some lengthy, but straightforward, algebra.

(iii) The statement follows from

$$
\lim _{\varepsilon \rightarrow 0} \sup _{\substack{\omega \mid=1 \\ \omega_{1} \neq 0}}\left|\hat{\hat{\Lambda}}\left(\lambda, \varepsilon \omega_{1}, \varepsilon \omega_{2}\right)-\hat{\Lambda}_{5}\left(\lambda, \omega_{1}, \omega_{2}\right)\right|=0 .
$$


In order to prove (6.36) we first remark that by (6.35) for $|\omega|=1$ the equation

$$
\operatorname{det}\left[\mathbf{F}\left(\lambda, \varepsilon \omega_{1}, y\right)-\mathrm{e}^{i \varepsilon \omega_{2}} \mathbf{I}\right]=0
$$

only admits a solution $y<\Lambda_{6}^{s}(\lambda)-\eta$ for $\varepsilon<\varepsilon_{0}$ if $\left|\omega_{1}\right|>\delta\left(\varepsilon_{0}\right)$. We may thus assume that $\left|\omega_{1}\right| \geqq \delta>0$. This implies that for $\varepsilon$ small enough,

$$
\left|\left(\frac{\omega_{2}}{\omega_{1}}\right)^{4}+I_{2}^{\mathbf{G}}(\lambda, y)\left(\frac{\omega_{2}}{\omega_{1}}\right)^{2}+I_{4}^{\mathbf{G}}(\lambda, y)\right| \leqq C \varepsilon .
$$

Taking into account the explicit form $I_{2}^{G}, I_{4}^{G}$ (see (6.31), (6.33)), one sees that, uniformly for all $|\omega|=1$ such that $\left|\omega_{1}\right| \geqq \delta>0, y$ must be close to a solution of

$$
\left(\frac{\omega_{2}}{\omega_{1}}\right)^{4}+I_{2}^{\mathbf{G}}(\lambda, y)\left(\frac{\omega_{2}}{\omega_{1}}\right)^{2}+I_{4}^{\mathbf{G}}(\lambda, y)=0
$$

This concludes the proof of (6.36).

Since $\hat{\Lambda}_{5}\left(\lambda, \omega_{1}, \omega_{2}\right)$ is continuous with respect to $\left(\omega_{1}, \omega_{2}\right)$ (see (6.32)), the infimum in (6.34) is attained at some $\left(\omega_{1 m}, \omega_{2 m}\right)$ and $\Lambda_{5}^{s}(\lambda)=$ $\hat{\Lambda}_{5}\left(\lambda, \omega_{1 m}, \omega_{2 m}\right)$. Two possibilities exist (recall that we assume that the stretch ratio $\lambda$ is fixed):

a) $\omega_{2 m} / \omega_{1 m}=0$ in which case, (6.38) implies

$$
I_{4}^{G}\left(\lambda, \Lambda_{5}^{s}\right)=0, \quad \frac{\omega_{2 m}}{\omega_{1 m}}=0 .
$$

b) $\omega_{2 m} / \omega_{1 m} \neq 0$. The continuity of $I_{2}^{\mathbf{G}}$ and $I_{4}^{\mathbf{G}}$ with respect to $\hat{\Lambda}_{5}$ dictates that in this case $\omega_{2 m} / \omega_{1 m}$ is a double root of (6.38) (and so is $-\left(\omega_{2 m} / \omega_{1 m}\right)$ ) since from (6.34) no real roots $\omega_{2} / \omega_{1}$ of the polynomial $\left(\frac{\omega_{2}}{\omega_{1}}\right)^{4}+$ $I_{2}^{\mathrm{G}}(\lambda, y)\left(\frac{\omega_{2}}{\omega_{1}}\right)^{2}+I_{4}^{\mathrm{G}}(\lambda, y)$ exist for $y<\Lambda_{5}^{s}(\lambda)$. Hence

$$
\left[I_{2}^{\mathrm{G}}\left(\lambda, \Lambda_{5}^{s}\right)\right]^{2}-4 I_{4}^{\mathrm{G}}\left(\lambda, \Lambda_{5}^{s}\right)=0, \quad\left(\frac{\omega_{2 m}}{\omega_{1 m}}\right)=\left(-\frac{1}{2} I_{2}^{\mathrm{G}}\left(\lambda, \Lambda_{5}^{s}\right)\right)^{1 / 2} .
$$

For a solution $\Lambda_{5}^{s}$ of $(6.40)$ to be acceptable one has to verify that $I_{2}^{\mathrm{G}}\left(\lambda, \Lambda_{5}^{s}\right)<0$. The desired $\Lambda_{5}^{s}(\lambda)$, which is defined in (6.29), is thus given by the minimum nonnegative root of (6.39), (6.40).

\subsection{Calculation of $\alpha_{M}^{s}(\lambda)$}

Besides $\Lambda(\lambda)$ and $\Lambda_{5}^{s}(\lambda)$ another quantity of interest in this example is $\alpha_{\mathbb{N}}^{s}(\lambda)$, which is defined by

$$
\alpha_{M}^{s}(\lambda)=\min _{\substack{|\boldsymbol{a}|=|\boldsymbol{b}|=1 \\ \boldsymbol{a}, \boldsymbol{b} \in \mathbb{R}^{N}}}\left(M^{s}(\lambda) \boldsymbol{a} \otimes \boldsymbol{b}, \boldsymbol{a} \otimes \boldsymbol{b}\right)
$$


where $M(\lambda)$ is the homogenized incremental moduli tensor of the composites principal solution whose components are given by (6.6).

From (6.41) one can easily deduce that $\alpha_{M}^{S}(\lambda)$ equals the minimum eigenvalue of the $2 \times 2$ matrix $M_{i j k l}(\lambda) b_{j} b_{l}$ over all possible unit vectors $b \in \mathbb{R}^{2}$. Assuming that $b=(\cos \phi, \sin \phi)$, we can thus obtain $\alpha_{\mathbb{M}}^{s}(\lambda)$ by the following expression

$$
\begin{aligned}
\alpha_{M}^{s}(\lambda)= & \min _{\phi \in[0, \pi / 2]} \frac{1}{2}\left\{\left[\left(M_{1111}+M_{2121}\right) \cos ^{2} \phi+\left(M_{1212}+M_{2222}\right) \sin ^{2} \phi\right]\right. \\
& -\left[\left[\left(M_{1111}-M_{2121}\right) \cos ^{2} \phi+\left(M_{1212}-M_{2222}\right) \sin ^{2} \phi\right]^{2}\right. \\
& \left.\left.+4\left(M_{1122}+M_{1221}\right)^{2} \sin ^{2} \phi \cos ^{2} \phi\right]^{1 / 2}\right\}
\end{aligned}
$$

The desired minimum can be found analytically by evaluating the righthand side of (6.42) at $\phi=0, \phi=\frac{\pi}{2}$ and comparing it with the extrema of the same function that occur in the interior points of $(0, \pi / 2)$. These extrema can be expressed in terms of $M_{i j k l}$, but their corresponding expressions are too cumbersome to be recorded here.

\subsection{Calculation of the critical stretch ratios $\lambda_{c}^{+}, \lambda_{c}^{-}$and $\lambda_{h}^{+}, \lambda_{h}^{-}$}

Another set of physically important quantities for the composite in this example are the zeros of $\Lambda(\lambda), \Lambda_{5}^{s}(\lambda)$ and $\alpha_{M}^{s}(\lambda)$ closest to $\lambda=1$. These zeros correspond to the stretch ratios associated with the first bifurcation and the first loss of ellipticity in the composite as the loading increases away from the stress-free state.

The roots of $\Lambda(\lambda)$ closest to $\lambda=1$ are denoted by $\lambda_{c}^{+}(>1)$ and $\lambda_{c}^{-}(<1)$. They respectively correspond to the onset of the first bifurcation instability in tension and compression as the stresses increase in absolute value.

From the defintion of $A(\lambda)$ in (6.7) one has the following method for the calculation of $\lambda_{c}^{+}$and $\lambda_{c}^{-}$:

$$
\begin{array}{ll}
\lambda_{c}^{+} \equiv \inf _{\lambda>1}\{\Lambda(\lambda)=0\}=\inf _{\omega_{1} \in \mathbb{R}^{+}}\left\{\hat{\lambda}_{c}^{+}\left(\omega_{1}\right)\right\}, & \hat{\lambda}_{c}^{+}\left(\omega_{1}\right) \equiv \inf _{\lambda>1}\left\{\hat{\Lambda}\left(\lambda, \omega_{1}\right)=0\right\}, \\
\lambda_{c}^{-}=\sup _{\lambda<1}\{\Lambda(\lambda)=0\}=\sup _{\omega_{1} \in \mathbb{R}^{+}}\left\{\hat{\lambda}_{c}^{-}\left(\omega_{1}\right)\right\}, & \hat{\lambda}_{c}^{-}\left(\omega_{1}\right) \equiv \sup _{\lambda<1}\left\{\hat{\Lambda}\left(\lambda, \omega_{1}\right)=0\right\} .
\end{array}
$$

It is tacitly assumed that $\lambda_{c}^{+}\left(\omega_{1}\right)$ and $\lambda_{c}^{-}\left(\omega_{1}\right)$ do exist for all $\omega_{1} \in \mathbb{R}^{+}$ and in view of (6.26)-(6.28) they are given by

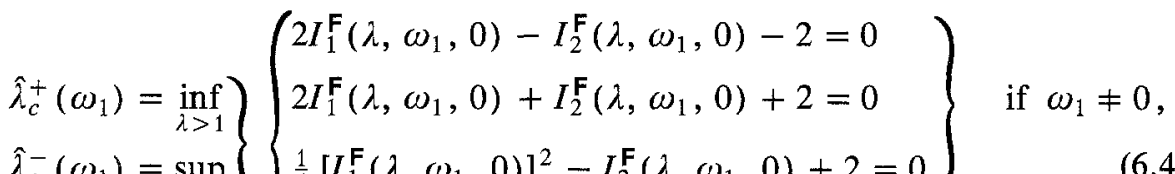

$$
\begin{aligned}
& \hat{\lambda}_{c}^{-}\left(\omega_{1}\right)=\sup _{\lambda<1}\left\{\left\{\begin{array}{l}
\frac{1}{4}\left[I_{1}^{\mathrm{F}}\left(\lambda, \omega_{1}, 0\right)\right]^{2}-I_{2}^{\mathrm{F}}\left(\lambda, \omega_{1}, 0\right)+2=0 \\
\left.\min L_{1212}^{\ell_{p}}(\lambda), L_{2222}(\lambda) \mid p=1,2, \ldots, n\right\}=0 \quad \text { if } \omega_{1}=0 .
\end{array}\right.\right.
\end{aligned}
$$


The inf and sup in (6.44) are expected to be min and max respectively because the $\lambda$ zeros of the expressions on the right-hand side of (6.44) are expected to be isolated zeros. The calculation of the lowest buckling loads $\lambda_{c}^{+}$ in tension and $\lambda_{c}^{-}$in compression is based on (6.43) $)_{2,3}$ and involves a simple numerical search of the respective extrema of $\hat{\lambda}_{c}^{+}\left(\omega_{1}\right)$ and $\hat{\lambda}_{c}^{-}\left(\omega_{1}\right)$ over an adequately large interval for $\omega_{1} \in \mathbb{R}^{+}$.

The zeros of $\Lambda_{5}^{s}(\lambda)$ closest to $\lambda=1$ are denoted by $\lambda_{h}^{+}(>1)$ and $\lambda_{h}^{-}(<1)$. They respectively correspond, according to Theorem 3.7 (vi), to the first loss of ellipticity in tension and compression of the homogenized incremental moduli of the composite's principal solution.

From the definition of $\lambda_{h}^{+}$and $\lambda_{h}^{-}$one has

$$
\lambda_{h}^{+} \equiv \inf _{\lambda>1}\left\{\Lambda_{5}^{s}(\lambda)=0\right\}, \quad \lambda_{\bar{h}}^{-} \equiv \sup _{\lambda<1}\left\{\Lambda_{5}^{s}(\lambda)=0\right\} .
$$

With the help of (6.39), (6.40), $\lambda_{h}^{+}$and $\lambda_{h}^{-}$are found to be

$$
\left.\begin{array}{l}
\lambda_{h}^{+}=\inf _{\lambda>1} \\
\lambda_{\bar{h}}^{-}=\sup _{\lambda<1}
\end{array}\right\}\left\{\begin{array}{l}
I_{4}^{\mathrm{G}}(\lambda, 0)=0, \\
{\left[I_{2}^{\mathrm{G}}(\lambda, 0)\right]^{2}-4 I_{4}^{\mathrm{G}}(\lambda, 0)=0 .}
\end{array}\right.
$$

As for (6.44), the inf and sup in (6.46) are expected to be attained at finite values of $\lambda$.

There is an interesting relation between $\lambda_{h}^{+}$and $\hat{\lambda}_{c}^{+}\left(\omega_{1}\right)$ and between $\lambda_{\bar{h}}^{-}$ and $\hat{\lambda}_{c}^{-}\left(\omega_{1}\right)$. By assuming the interchangeability of $\lim _{\varepsilon \rightarrow 0^{+}}$and $\inf _{\omega_{2} \in[0,2 \pi)}$ in $\hat{\hat{\Lambda}}\left(\lambda, \varepsilon \omega_{1}, \varepsilon \omega_{2}\right)$ from the definitions (6.8) and (6.9) as well as from (6.29), (6.30), (6.34), (6.43) and (6.45) one obtains

$$
\lambda_{h}^{+}=\hat{\lambda}_{c}^{+}\left(0^{+}\right)=\lim _{\varepsilon \rightarrow 0^{+}} \hat{\lambda}_{c}^{+}\left(\varepsilon \omega_{1}\right), \quad \lambda_{h}^{-}=\hat{\lambda}_{c}^{-}\left(0^{+}\right)=\lim _{\varepsilon \rightarrow 0^{+}} \hat{\lambda}_{c}^{-}\left(\varepsilon \omega_{1}\right) .
$$

This property is verified in all the numerical calculations done for the layered composite. The zeros of $\alpha_{\mathbb{M}}^{S}(\lambda)$ closest to $\lambda=1$ are denoted by $\lambda_{H}^{+}(>1)$ and $\lambda_{H}(<1)$. By definition,

$$
\lambda_{H}^{+} \equiv \inf _{\lambda>1}\left\{\alpha_{M}^{\mathrm{s}}(\lambda)=0\right\}, \quad \lambda_{H}^{-} \equiv \sup _{\lambda<1}\left\{\alpha_{M}^{s}(\lambda)=0\right\} .
$$

As expected from Theorem 3.7, $\lambda_{H}^{+}=\lambda_{h}^{+}, \lambda_{H}^{-}=\lambda_{h}^{-}$, a result to be verified constructively in the sequel.

By making use of (6.42) into (6.48) one finds

$$
\lambda_{H}^{+}=\inf _{\lambda<1} I_{4}^{H}(\lambda)=0, \quad \lambda_{H}^{-} \equiv \sup _{\lambda>1}\left[I_{2}^{H}(\lambda)\right]^{2}-4 I_{4}^{H}(\lambda)=0
$$

where the quantities $I_{2}^{H}, I_{4}^{H}$ are given in terms of $M_{i j k l}(\lambda)$ by

$$
\begin{aligned}
I_{2}^{H}(\lambda) \equiv & {\left[M_{1111}(\lambda) M_{2222}(\lambda)+M_{1212}(\lambda) M_{2121}(\lambda)\right.} \\
& \left.-\left(M_{1122}(\lambda)+M_{2222}(\lambda)\right)^{2}\right]\left[M_{1212}(\lambda) M_{2222}(\lambda)\right]^{-1}, \\
I_{4}^{H}(\lambda) \equiv & {\left[M_{1111}(\lambda) M_{2121}(\lambda)\right]\left[M_{1212}(\lambda) M_{2222}(\lambda)\right]^{-1} . }
\end{aligned}
$$


The homogenized moduli components $M_{i j k l}(\lambda)$ are given by (6.6). Note that in view of the assumption (E) (strong ellipticity of each layer) $L_{1212}^{\ell_{p}}(\lambda)>0$ and $L_{2222}^{\ell_{p}}(\lambda)>0$ for $p=1, \ldots, n$, which ensures from (6.6) that $M_{1212}(\lambda)>0$ and $M_{2222}(\lambda)>0$ and hence $I_{2}^{H}(\lambda), I_{4}^{H}(\lambda)$ are always defined.

A straightforward calculation of the invariants $I_{2}^{\mathrm{G}}(\lambda, 0)$ and $I_{4}^{\mathrm{G}}(\lambda, 0)$ in (6.33) by using (6.31) and (6.6) gives

$$
I_{2}^{H}(\lambda)=I_{2}^{\mathrm{G}}(\lambda, 0), \quad I_{4}^{H}(\lambda)=I_{4}^{\mathrm{G}}(\lambda, 0) .
$$

Comparing (6.46) to (6.49) in the light of (6.50) one deduces that

$$
\lambda_{h}^{+}=\lambda_{H}^{+}, \quad \lambda_{h}^{-}=\lambda_{H}^{-} .
$$

This result is an independent proof for the layered composite at hand of the general result in Theorem 3.7 concerning the zeros of $\Lambda_{5}^{s}(\lambda)$.

As expected from the definitions (6.43), (6.45) and in view of the properties (6.47), (6.51), the following relations hold for the zeros of $\Lambda(\lambda), \Lambda_{5}^{s}(\lambda)$ and $\alpha_{M}^{\mathrm{s}}(\lambda)$ :

$$
1<\lambda_{c}^{+} \leqq \hat{\lambda}_{c}^{+}\left(0^{+}\right)=\lambda_{h}^{+}=\lambda_{H}^{+}, \quad 1>\lambda_{c}^{-} \geqq \hat{\lambda}_{c}^{-}\left(0^{+}\right)=\lambda_{h}^{-}=\lambda_{H}^{-} .
$$

All the results given thus far for the two-dimensional layered composite under axial stretching are valid for any choice of nonlinearly elastic material satisfying $(E)$ for each layer. In practice, the requirement $(E)$ of strong pointwise ellipticity is not essential, for all the important instability phenomena occur well below the stress levels corresponding to the loss of ellipticity of the weakest layer (see [TM 85]).

\subsection{Example for a particular energy density $W$}

For the numerical application that accompanies the example of the layered composite, a particular isotropic polyconvex (see [B 77]) material employed by OGDEN [O 84] is used. If $v_{i}$ denotes the singular values of the deformation gradient $F$, its strain-energy density $W$ is given by

$$
W\left(v_{1}, v_{2}\right)=\frac{\mu}{2}\left[v_{1}^{2}+v_{2}^{2}-2-2 \ln \left(v_{1} v_{2}\right)\right]+\frac{\kappa}{2}\left[v_{1} v_{2}-1\right]^{2} .
$$

The constants $\mu$ and $\kappa$ are the initial shear and bulk moduli of the material which are related to its initial Young's modulus, $E$, and Poisson's ratio, $v$, by

$$
\mu=\frac{E}{2(1+v)}, \quad \kappa=\frac{v E}{(1+v)(1-2 v)} .
$$

It is not difficult to see that as $v \rightarrow \frac{1}{2}$ in the incompressible limit, $W \rightarrow \frac{1}{2} \mu\left(v_{1}^{2}+v_{2}^{2}-2\right), v_{1} v_{2}=1$, which is the two-dimensional form of the well-known Mooney-Rivlin material, a popular first approximation for modeling rubber. Therefore the energy density in (6.52) satisfies all the requirements 
for a physically reasonable model of a rubber-type material. Moreover $W>0$ for all $\left(v_{1}, v_{2}\right) \neq(1,1)$ and $W \rightarrow \infty$ when either $\|F\| \rightarrow \infty$ or $\operatorname{det} F \rightarrow 0$. The last condition, although physically important, is not compatible with the growth assumptions for $W$ adopted in (2.2), (2.3). In the ensuing calculations, however, $\operatorname{det} F$ is bounded away from zero (i.e., $\operatorname{det} F \geqq \delta>0$ ) and hence for the deformations of interest the growth conditions are satisfied.

The principal Cauchy stresses $\sigma_{11}, \sigma_{22}$ for this material, calculated by using (6.52), are

$$
\sigma_{11}=\frac{\mu}{v_{1} v_{2}}\left(v_{1}^{2}-1\right)+\kappa\left(v_{1} v_{2}-1\right), \quad \sigma_{22}=\frac{\mu}{v_{1} v_{2}}\left(v_{2}^{2}-1\right)+\kappa\left(v_{1} v_{2}-1\right) .
$$

They vanish at $\left(v_{1}, v_{2}\right)=(1,1)$.

Since within each layer the deformation gradient tensor is $F=F(\lambda)=$ $\operatorname{diag}\left[\lambda_{1}, \lambda_{2}\right]$, it follows that $v_{i}=\lambda_{i}$. In view of the assumption that the lateral stress in each layer is $\sigma=0$ (i.e., $\sigma_{22}=0$ in each layer), one finds from (6.54) $)_{2}$ that $\lambda_{2}$ in each layer is given in terms of $\lambda_{1}=\lambda$ and the material constants $\mu$ and $\kappa$ by

$$
\lambda_{2}=\frac{\lambda+\left[\lambda^{2}+4(\mu / \kappa)\left(\lambda^{2}+\mu / \kappa\right)\right]^{1 / 2}}{2\left(\lambda^{2}+\mu / \kappa\right)} .
$$

The nonzero components of the incremental moduli tensor $L_{i j k l}$ for the material considered in (6.52) are

$$
\begin{aligned}
& L_{1111}=\mu\left(1+\frac{1}{v_{1}^{2}}\right)+\kappa v_{2}^{2}, \quad L_{1122}=\kappa\left(2 v_{1} v_{2}-1\right)=L_{2211}, \\
& L_{2222}=\mu\left(1+\frac{1}{v_{2}^{2}}\right)+\kappa v_{1}^{2}, \quad L_{1212}=\mu=L_{2121}, \\
& L_{1221}=\frac{\mu}{v_{1} v_{2}}+\kappa\left(1-v_{1} v_{2}\right)=L_{2112} .
\end{aligned}
$$

The incremental moduli at $\left(v_{1}, v_{2}\right)=(1,1)$ are equal to those of isotropic linear elasticity, as can be easily checked from (6.56).

The results of the numerical calculations using the material model in (6.52) are depicted in Figs. 6.2-6.6. In all these calculations the value $E=1$ was adopted. The dependence of the various dimensionless coercivity constants $\Lambda / E, \Lambda_{5}^{s} / E, \Lambda_{6}^{s} / E$ and $\alpha_{M}^{s} / E$ on the stretch ratio $\lambda$ for a composite symmetric about $x_{1}$ under compression is plotted in Fig. 6.2. In this case $\stackrel{a}{E}=\stackrel{c}{E}=0.1 E$, $\stackrel{b}{E}=1.5 E, \stackrel{a}{v}=\stackrel{c}{v}=0.33, \stackrel{b}{v}=0.48, \stackrel{a}{H}=\stackrel{c}{H}=0.45, \stackrel{b}{H}=0.1$. Only values of $\lambda$ for which $\Lambda(\lambda)>0$ have been considered. As expected from the theory in Section 3 the conditions $\Lambda(\lambda) \leqq \Lambda_{5}^{s}(\lambda) \leqq \alpha_{\mathbb{N}}^{s}(\lambda)$ are obviously satisfied. In order to calculate $\Lambda(\lambda)$ from $\hat{\Lambda}\left(\lambda, \omega_{1}\right)$ (see definition in (6.7)) the interval $(0,7)$ is considered instead of $\mathbb{R}^{+}$. In all the calculations reported here, the value of $\omega_{1}$ which minimizes $\hat{\Lambda}\left(\lambda, \omega_{1}\right)$ always falls in this interval. For 


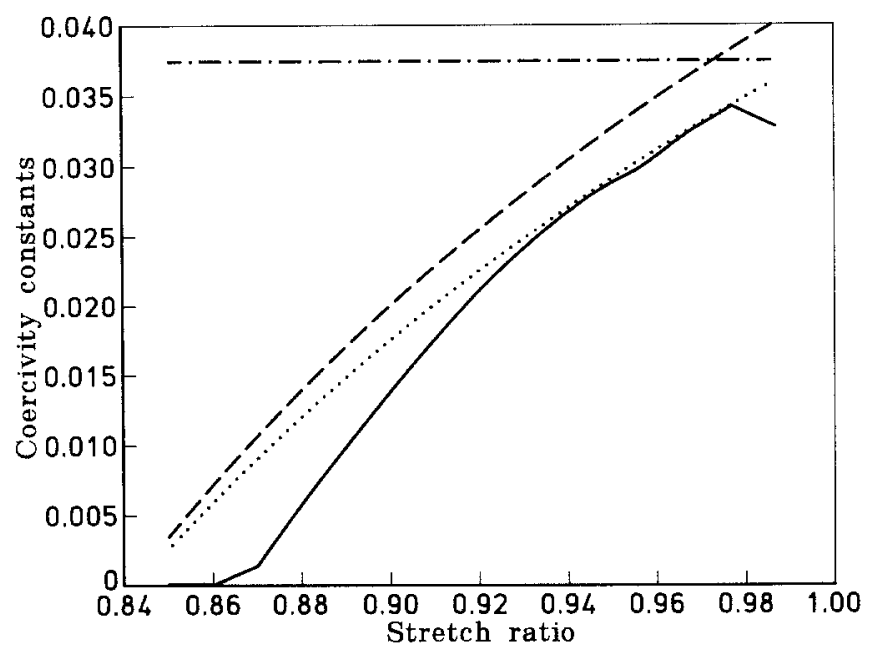

Fig. 6.2. Dependence of the dimensionless coercivity constants $\Lambda / E$ (solid line), $\Lambda_{5}^{s} / E$ (dotted line), $\alpha_{M}^{s} / E$ (dashed line) and $A_{6}^{s} / E$ (dash-dot line) on the stretch ratio $\lambda$ for a composite (with a unit cell symmetric about $x_{1}$ ) under compression. The calculations are based upon the following material constants: layer moduli $\stackrel{a}{E}=\stackrel{c}{E}=0.1 E$, $\stackrel{b}{E}=1.5 E$; layer Poisson ratios $\stackrel{a}{v}=\stackrel{c}{v}=0.33, \stackrel{b}{v}=0.48$; layer thicknesses $\stackrel{a}{H}=\stackrel{c}{H}=0.45$, $\stackrel{b}{H}=0.1$.

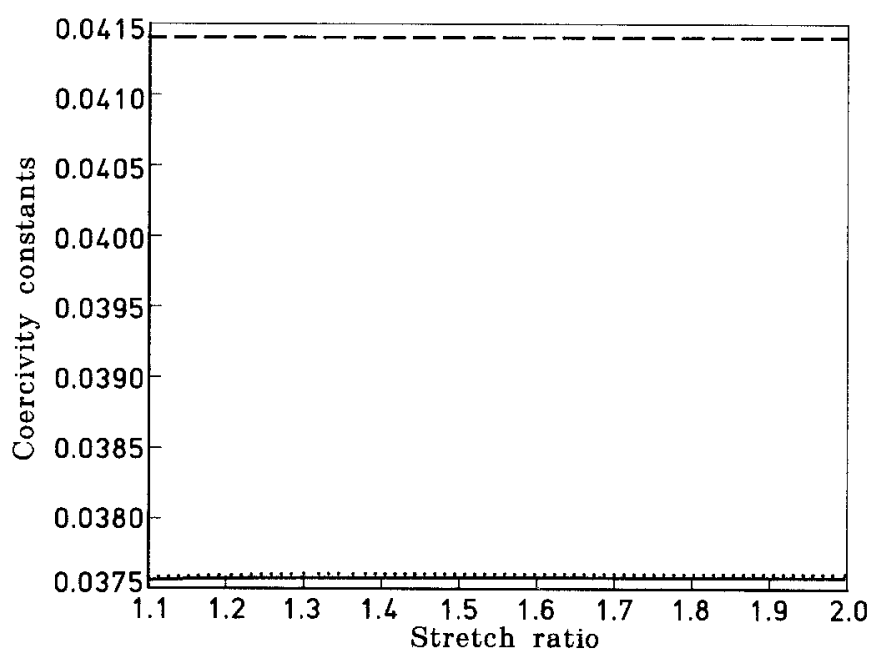

Fig. 6.3. Dependence of the dimensionless coercivity constants $\Lambda / E$ (solid line), $\Lambda_{5}^{s} / E$ (dotted line), $\alpha_{\mathrm{M}}^{s} / E$ (dashed line) and $\Lambda_{6}^{s} / E$ (dash-dot line) on the stretch ratio $\lambda$ for a composite (with a unit cell symmetric about $x_{1}$ ) under tension. Note that in this case the curves for $\Lambda / E, \Lambda_{5}^{s} / E$ and $\Lambda_{6}^{s} / E$ coincide. The calculations are based upon the following material constants: layer moduli $\stackrel{a}{E}=\stackrel{c}{E}=0.1 E, \stackrel{b}{E}=1.5 E$; layer Poisson ratios $\stackrel{a}{v}=\stackrel{c}{v}=0.33, \stackrel{b}{v}=0.48$; layer thicknesses $\stackrel{a}{H}=\stackrel{c}{H}=0.45, \stackrel{b}{H}=0.1$. 
$\lambda<0.94$ approximately, $\Lambda(\lambda)<\Lambda_{5}^{s}(\lambda)$, which indicates the existence of a finite wave number $\omega_{1 m}>0$ corresponding to $\Lambda(\lambda)$ (i.e., $\Lambda(\lambda)=\hat{\Lambda}\left(\lambda, \omega_{1 m}\right)$ ). It is also worth mentioning that for all the investigated values of $\lambda$ and $\omega_{1}$ the eigenmode corresponding to $\hat{\Lambda}\left(\lambda, \omega_{1}\right)$ (see (6.26), (6.27)) is either a symmetric or an antisymmetric one, i.e., $\omega_{2 m}=0$ or $\omega_{2 m}=\pi$ with the symmetric case occurring most frequently.

The graphs of $\Lambda / E, \Lambda_{5}^{s} / E, \Lambda_{6}^{s} / E$ and $\alpha_{M}^{s} / E$ for the same composite as in Fig. 6.2 now subjected to tension, are depicted in Fig. 6.3. In this case $\hat{\Lambda}\left(\lambda, \omega_{1}\right)$ has its minimum at $\omega_{1}=0$ for all values of $\lambda$, and is equal to the initial shear modulus (see (6.53) $)_{2}$ of the weakest layer, i.e., $\Lambda(\lambda)=\Lambda_{5}^{s}(\lambda)=$ $\Lambda_{6}^{s}(\lambda)=\hat{\Lambda}(\lambda, 0,0)=\stackrel{a}{\mu}=\stackrel{c}{\mu}=0.037594 E$. The value of $\alpha_{M}^{s}(\lambda)$ is also constant independent of $\lambda$, and is equal to the homogenized shear modulus of the composite $M_{2121}=0.0414 E$.

The graphs in Fig. 6.3 are typical for all the composites under tension and hence the results from only one composite under tension are presented here.

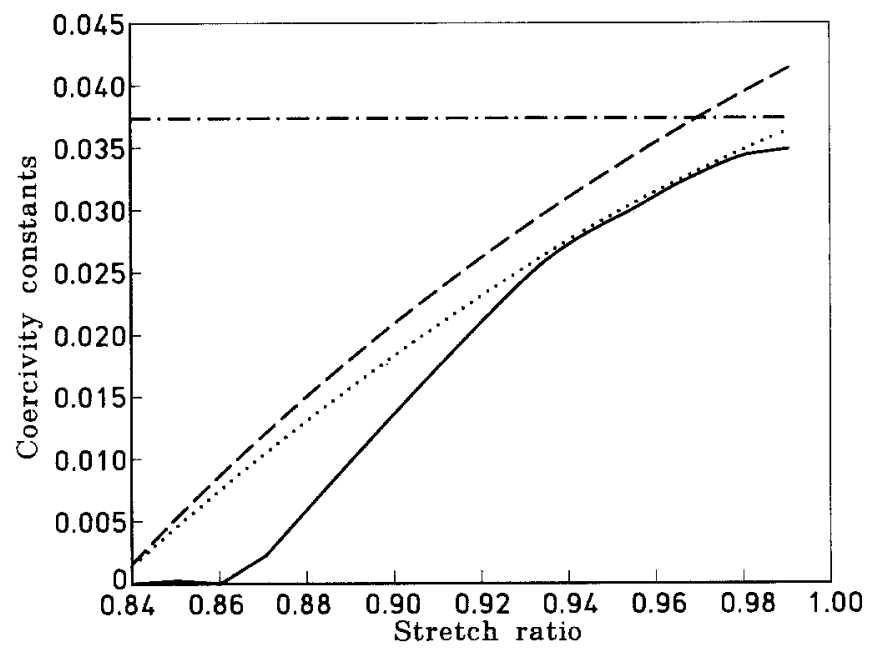

Fig. 6.4. Dependence of the dimensionless coercivity constants $\Lambda / E$ (solid line), $\Lambda_{5}^{s} / E$ (dotted line), $\alpha_{\mathrm{M}}^{s} / E$ (dashed line) and $\Lambda_{6}^{s} / E$ (dash-dot line) on the stretch ratio $\lambda$ for a composite (with an asymmetric unit cell) under compression. The calculations are based upon the following material constants; layer moduli $\stackrel{a}{E}=\stackrel{c}{E}=0.1 E, \stackrel{b}{E}=1.5 E$; layer Poisson ratios $\stackrel{a}{v}=0.22, \stackrel{b}{v}=0.48, \stackrel{c}{v}=0.33$; layer thicknesses $\stackrel{a}{H}=\stackrel{c}{H}=0.45, \stackrel{b}{H}=0.1$.

The graphs $\Lambda / E, \Lambda_{5}^{s} / E, \Lambda_{6}^{s} / E$ and $\alpha_{M}^{s} / E$ for two other composites, this time with nonsymmetric material properties with respect to the $x_{2}$ axes, are depicted in Fig. 6.4 and Fig. 6.5. More specifically, Fig. 6.4 corresponds to a composite with nonsymmetric properties under compression with $\stackrel{a}{E}=\stackrel{c}{E}=0.1 E$, $\stackrel{b}{E}=1.5 E, \stackrel{a}{v}=0.22, \stackrel{c}{v}=0.48, \stackrel{c}{v}=0.33 ; \stackrel{a}{H}=\stackrel{c}{H}=0.45, \stackrel{c}{H}=0.1$. Notice that in this case the results are very similar to those for the symmetric composite in Fig. 6.2. 


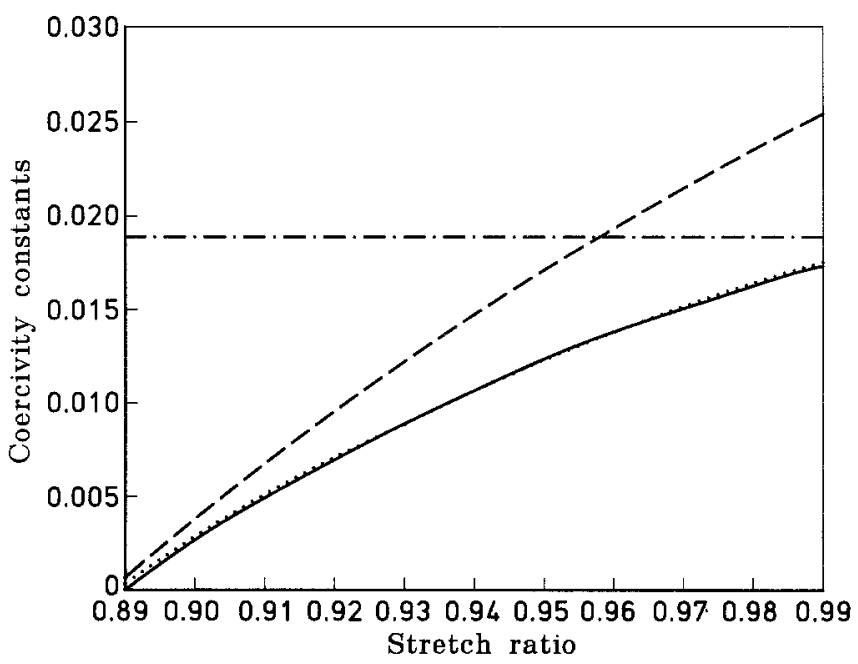

Fig. 6.5. Dependence of the dimensionless coercivity constants $\Lambda / E$ (solid line), $\Lambda_{5}^{s} / E$ (dotted line), $\alpha_{\mathrm{M}}^{s} / E$ (dashed line) and $\Lambda_{6}^{s} / E$ (dash-dot line) on the stretch ratio $\lambda$ for a composite (with a asymmetric unit cell) under compression. Note that for a large range of the stretch ratio the curves for $\Lambda / E$ and $\Lambda_{5}^{s} / E$ coincide (at least numerically). The calculations are based upon the following materials constants: layer moduli $\stackrel{a}{E}=\stackrel{b}{E}=0.1 E, \stackrel{c}{E}=0.0 E$; layer Poisson ratios $\stackrel{a}{v}=\stackrel{c}{v}=0.33, \stackrel{b}{v}=0.48$; layer thicknesses $\stackrel{a}{H}=\stackrel{c}{H}=0.45, \stackrel{b}{H}=0.1$.

The results in Fig. 6.5 correspond to a composite with nonsymmetric properties under compression with $\stackrel{a}{E}=0.1 E, \stackrel{b}{E}=1.5 E, \stackrel{c}{E}=0.05 E ; \stackrel{a}{v}=\stackrel{c}{v}=0.33$, $\stackrel{b}{v}=0.48 ; \stackrel{a}{H}=\stackrel{c}{H}=0.45, \stackrel{b}{H}=0.1$. The main difference in this case as compared to Fig. 6.2 and Fig. 6.4 is that $\Lambda(\lambda)=\Lambda_{5}^{s}(\lambda)$ for the investigated range of the stretch ratio $\lambda$.

It is worth noticing that in all the calculations for compression, $\Lambda_{5}^{s}<\Lambda_{6}^{s}$ for the range of $\lambda$ investigated. That $\Lambda_{6}^{s}$ is independent of $\lambda$ is a particular feature of the example taken for $W$ in (6.52) (see also (6.56) where $\left.L_{1212}(\lambda)=\mu<L_{2222}(\lambda)\right)$.

Finally of interest are the critical stretch ratios corresponding to the first bifurcation $\lambda_{c}^{-}$(see (6.43)) and to the first loss of ellipticity of the principal solution's homogenized moduli $\lambda_{H}=\lambda_{\bar{h}}$ (see (6.48)) for the material model investigated here. Without loss of generality, only composites with two different materials per unit cell are considered. More specifically, the two materials are those employed in the calculations for Fig. 6.2, i.e., $\stackrel{a}{E}=0.1 E$, $\stackrel{b}{E}=1.5 E ; \stackrel{a}{v}=0.33, \stackrel{b}{v}=0.48$. The ratio $\stackrel{b}{H}$ denotes the volume fraction of the stiffer material in the composite. In Fig. 6.6 is depicted the dependence of the critical stretch ratios $\lambda_{c}^{-}$and $\lambda_{\bar{H}}$ on the ratio $H_{\text {. }}$

Note that for $\stackrel{b}{H}>0.15$ (approximately) one has $\lambda_{c}^{-}=\lambda_{H}=\hat{\lambda}_{c}^{-}\left(0^{+}\right)$, which implies that the first instability encountered in the composite as the 


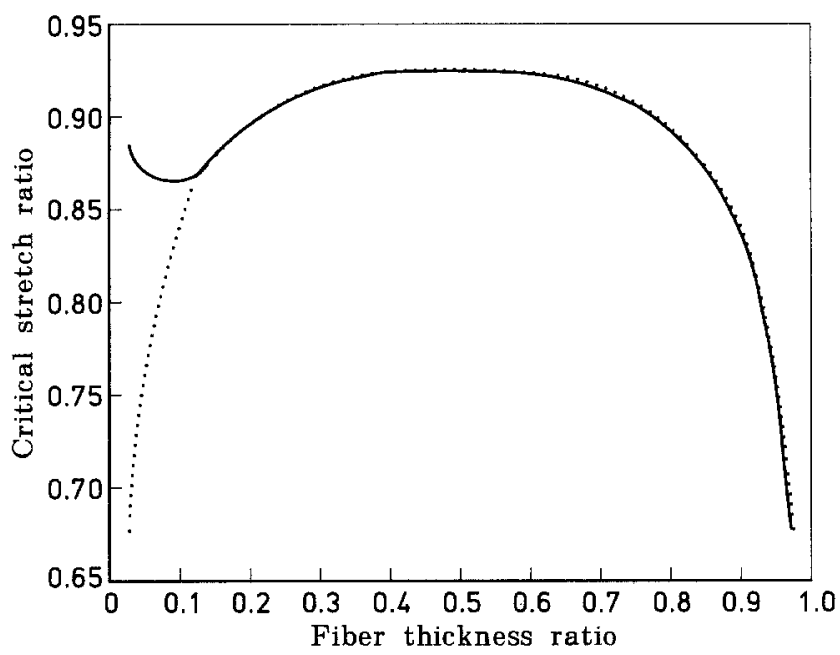

Fig. 6.6. Dependence of the the critical stretch ratios $\lambda_{c}^{-}$(solid line), $\lambda_{h}^{-}$(dotted line), on fiber thickness ratio $\stackrel{a}{H}$ for a composite (with a unit cell symmetric about $x_{1}$ ) under compression. The calculations are based upon the following material constants: layer moduli $\stackrel{a}{E}=\stackrel{c}{E}=0.1 E, \stackrel{b}{E}=1.5 E$; layer Poisson ratios $\stackrel{a}{v}=\stackrel{c}{v}=0.33, \stackrel{b}{v}=0.48$; layer thicknesses $\stackrel{a}{H}=\stackrel{c}{H}$.

compressive stresses increase is a long wavelength instability with $\omega_{1} \rightarrow 0^{+}$. Only for relatively thin stiff fibers in the composite $(\stackrel{b}{H}<0.15)$ the first instability in the composite corresponds to a fiber buckling with $\omega_{1 c}>0$ where $\lambda_{c}^{-} \equiv \hat{\lambda}_{c}\left(\omega_{1 c}\right)$. It is found that the thinner the fiber, the larger the critical value $\omega_{1 c}$ (corresponding to the maximum of $\hat{\lambda}_{c}^{-}\left(\omega_{1 c}\right)$ over $\mathbb{R}^{+}$) becomes. In the numerical calculations reported in Fig. 6.6 the interval $(0,24)$ always contains the maximum $\omega_{1}$ of $\lambda_{c}\left(\omega_{1}\right)$. The facts that for $\stackrel{b}{H}<0.15$, $\lambda_{c}^{-}>\lambda_{H}$ and that $\left.\lambda_{c}^{-} \stackrel{b}{H}\right) \rightarrow 1$ as $\stackrel{b}{H} \rightarrow 0$ are expected physically, since the thinner the stiff fiber becomes, the lower the required stress to buckle it.

It is interesting to notice that although the two constituent materials of the composite are strongly elliptic at any level of deformation, in view of the polyconvexity of the assumed energy density in (6.52), the resulting homogenized composite can lose ellipticity at fairly low strains, e.g., $\lambda=0.927$ for a ratio $\stackrel{b}{H}=0.5$ of the two constituents. Similar results were found in [TM 85] for the incompressible case.

\section{References}

[AT 81] R. Abeyaratne \& N. Triantafyllidis, The emergence of shear bands in plane strain, Int. J. Solids Structures, 17 (1981), pp. 1113-1134.

[AT 84] R. Abeyaratne \& N. TRIANTAFYllidis, An investigation of localization in a porous elastic material using homogenization theory, J. Appl. Mech, 51 (1984), pp. $481-486$. 
[AF 84] E. ACERBI \& N. Fusco, Semicontinuity problems in the calculus of variations, Arch. Rational Mech. Anal., 86 (1984), pp. 125-145.

[A 84] H. Artouch, Variational Convergence of Functions and Operators, Pitman, London (1984).

[B 77] J. M. BALL, Convexity conditions and existence theorems in nonlinear elasticity, Arch. Rational Mech. Anal., 63 (1977), pp. 337-403.

[BLP 78] A. Bensoussan, J.-L. Lions \& G. Papanicolaou, Asymptotic Analysis for Periodic Structures, North-Holland, Amsterdam (1978).

[Br 85] A. BRAIDES, Homogenization of some almost periodic coercive functional, Rend. Accad. Naz. XL, 9 (1985), pp. 313-322.

[DM 81] G. DalMaso \& L. Modica, A general theory of variational functionals, in: "Topics in Functional Analysis" (1980-1981), eds. F. STrocChI, E. Zarantonelio, E. DeGiorgi, E. DalMaso \& L. Modica, Sc. Norm. Sup., Pisa (1981), pp. 149-221.

[D 75] E. DiGiongI, Sulla convergenza di alcune successioni di integrali del tipo dell'area, Rend. Matematica, 8 (1975), pp. 277-294.

[D 79] E. DeGiongI, Convergence problems for functions and operators, Proc. Int. Meeting on "Recent Methods in Nonlinear Analysis", Rome (1978), eds. E. DeGiorgi, E. Magenes \& U. Mosco, Pitagora, Bologna (1979), pp. $131-188$.

[DD 83] E. DeGiorgi \& G. DalMaso, F-convergence and the calculus of variations, in "Mathematical theories of optimization", eds. J. P. CECCONI \& T. ZolezzI, Lect. Notes Math., 979 Springer, Berlin, Heidelberg, New York (1983), pp. $121-143$.

[F 83] G. FrancForT, Homogenization and linear thermoelasticity, SIAM J. Math. Anal., 14 (1983), pp. 696-708.

[FM 91] G. FRANCFORT \& F. MURAT, personal communication.

[GMT 90] G. Geymonat, S. Müller \& N. Triantafyllidis, Quelques remarques sur l'homogénéisation des matériaux élastiques nonlinéaires, C. R. Acad. Sci. Paris, Serie I, 311 (1990), pp. $911-916$.

[GT 59] A. N. GeNT \& A. G. Thomas, Mechanics of foamed elastic materials, Rubber Chem. Tech., 36 (1959), pp. 597-610.

[HS 62] Z. HASHIN \& S. SHTRIKMAN, On some variational principles in anisotropic and nonhomogeneous elasticity, J. Mech. Physics Solids, 10 (1962), pp. 335-342.

[HI 82] N. C. Hiryard (Ed.), Mechanics of Cellular Plastics, Appl. Science Publ., London (1982).

[H 63] R. HIL, Elastic properties of reinforced solids: Some theoretical principles, J. Mech. Phys. Solids, 11 (1963), pp. 357-372.

[H 65] R. HILL, A self-consistent mechanics of composite materials, J. Mech. Phys. Solids, 13 (1965), pp. 213-222.

[KS 86] R. V. Kohn \& G. STRANG, Optimal design and relaxation of variational problems, parts I-III, Comm. Pure Appl. Math., 39 (1986), pp. 113-137, $138-182,353-377$.

[K 67] E. KRÖNER, Elastic moduli of perfectly disordered composite materials, J. Mech. Phys. Solids, 15 (1967), pp. 319-340.

[LD 87] H. LEDRET, An example of $H^{1}$-unboundedness of solutions to strongly elliptic systems of PDEs in a laminated geometry, Proc. Royal Soc. Edinburgh, 105 A (1987), pp. $77-82$.

[Ma 78] P. MARCELlINI, Periodic solutions and homogenization of nonlinear variational problems, Ann. Mat. Pura Appl., 117 (1978), pp. 139-152. 
[M 52] C. B. Morrey, Jr., Quasi-convexity and lower semicontinuity of multiple integrals, Pacific J. Math., 2 (1952), pp. 25-53.

[M 66] C. B. Morrey, Jr., Multiple Integrals in the Calculus of Variations, Springer, Berlin, Heidelberg, New York (1966).

[Mü 87] S. MüLLER, Homogenization of nonconvex integral functionals and cellular elastic materials, Arch. Rational Mech. Anal., 99 (1987), pp. 189-212.

[O 84] R. OGden, Nonlinear Elastic Deformations, Wiley, New York (1984).

[PC 89] P. PONTE-CASTENEDA, The overall constitutive behaviour of nonlinearily elastic composites, Proc. Roy. Soc. London, 422A (1989), pp. 147-171.

RS 79] M. ReEd \& B. Simon, Methods of Modern Mathematical Physics, Vol. IV, Academic Press, New York (1979).

[SH SP 89] J. Sanchez-Hubert \& E. Sanchez-Palencia, Vibration and Coupling of Continuous Systems. Asymptotic Methods, Springer, Berlin, Heidelberg, New York. (1989).

[SP 74] E. SANChez-PALENCIA, Comportement local et macroscopique d'un type de milieux physiques hétérogènes, Int. J. Eng. Sci., 12 (1974), pp. 331-351.

[SP 80] E. SancheZ-PALENCIA, Nonhomogeneous Media and Vibration Theory, Lect. Notes Physics, 127, Springer, Berlin, Heidelberg, New York (1980).

[SP 86] E. Sanchez-PALENCIA, Homogenization in mechanics - A survey of solved and open problems, Rend. Sem. Mat. Univ. Politecn. Torino, 44 (1986), pp. $1-45$.

[S 68] S. SPANGolo, Sulla convergenza di soluzioni di equazioni paraboliche ed ellitiche, Ann. Sc. Norm. Sup. Pisa, 22 (1968), pp. 571-597.

[ST 70] E. SteIN, Singular Integrals and Differentiability Properties of Functions, Princeton University Press, Princeton (1970).

[Sv 92] V. Šverák, Rank-one convexity does not imply quasiconvexity, Proc. Roy. Soc. Edinburgh, 120 (1992), pp. 185-189.

[TW 87] D. R. S. TALboT \& J. R. Willis, Bounds and Self-Consistent Estimates for the Overall Properties of Nonlinear Composites, IMA J. Appl. Math., 39 (1987), pp. 215-240.

[TM 85] N. TRIANTAFYllidis \& B. N. MAKER, On the comparison between microscopic and macroscopic instability mechanisms in a class of fiber-reinforced composites, J. Appl. Mech., 52 (1985), pp. 794-800.

[W 81] J. R. WIILIS, Variational and related methods for the overall properties of Composites, in "Advances in Applied Mechanics", 21, Academic Press, New York (1981), pp. 1-78.

Laboratoire de Mécanique et Technologie

E.N.S. de Cachan

61, avenue du Président Wilson

F-94235 Cachan Cedex, France

Institut für Angewandte Mathematik

Universität Bonn, Beringstrasse 4

D-53115 Bonn

and

Department of Aerospace Engineering

The University of Michigan

Aerospace Engineering Building

Ann Arbor, Michigan 48109-2140

(Received August 10, 1992) 